\title{
Altering the availability or proximity of food, alcohol, and tobacco products to change their selection and consumption (Review)
}

Hollands GJ, Carter P, Anwer S, King SE, Jebb SA, Ogilvie D, Shemilt I, Higgins JPT, Marteau TM

Hollands GJ, Carter P, Anwer S, King SE, Jebb SA, Ogilvie D, Shemilt I, Higgins JPT, Marteau TM.

Altering the availability or proximity of food, alcohol, and tobacco products to change their selection and consumption.

Cochrane Database of Systematic Reviews 2019, Issue 9. Art. No.: CD012573.

DOI: 10.1002/14651858.CD012573.pub3.

www.cochranelibrary.com

Altering the availability or proximity of food, alcohol, and tobacco products to change their selection and consumption (Review) 
HEADER

ABSTRACT

PLAIN LANGUAGE SUMMARY

SUMMARY OF FINDINGS

BACKGROUND

OBJECTIVES

METHODS

Figure 1.

RESULTS

Figure 2.

Figure 3.

Figure 4.

Figure 5.

Figure 6.

DISCUSSION

AUTHORS' CONCLUSIONS

ACKNOWLEDGEMENTS

REFERENCES

CHARACTERISTICS OF STUDIES

ADDITIONAL TABLES

APPENDICES

WHAT'S NEW

CONTRIBUTIONS OF AUTHORS

DECLARATIONS OF INTEREST

SOURCES OF SUPPORT

DIFFERENCES BETWEEN PROTOCOL AND REVIEW

NOTES

INDEX TERMS

\section{TABLE OF CONTENTS}

1

1

2

4

7

9

9

12

17

18

22

23

25

26

27

29

31

32

42

78

81

92

92

93

93

93

93

94 
[Intervention Review]

\section{Altering the availability or proximity of food, alcohol, and tobacco products to change their selection and consumption}

Gareth J Hollands ${ }^{1}$, Patrice Carter ${ }^{2}$, Sumayya Anwer ${ }^{3}$, Sarah E King ${ }^{1}$, Susan A Jebb 4 , David Ogilvie ${ }^{5}$, Ian Shemilt ${ }^{6}$, Julian P T Higgins ${ }^{3}$, Theresa M Marteau ${ }^{1}$

${ }^{1}$ Behaviour and Health Research Unit, University of Cambridge, Cambridge, UK. ${ }^{2}$ Centre for Outcomes Research and Effectiveness, University College London, London, UK. ${ }^{3}$ Population Health Sciences, Bristol Medical School, University of Bristol, Bristol, UK. ${ }^{4}$ Nuffield Department of Primary Care Health Sciences, University of Oxford, Oxford, UK. ${ }^{5}$ MRC Epidemiology Unit, University of Cambridge, Cambridge, UK. ${ }^{6}$ EPPI-Centre, University College London, London, UK

Contact address: Gareth J Hollands, Behaviour and Health Research Unit, University of Cambridge, Forvie Site, Robinson Way, Cambridge, CB2 0SR, UK. gareth.hollands@medschl.cam.ac.uk.

Editorial group: Cochrane Public Health Group.

Publication status and date: Edited (no change to conclusions), published in Issue 9, 2019.

Citation: Hollands GJ, Carter P, Anwer S, King SE, Jebb SA, Ogilvie D, Shemilt I, Higgins JPT, Marteau TM. Altering the availability or proximity of food, alcohol, and tobacco products to change their selection and consumption. Cochrane Database of Systematic Reviews 2019, Issue 9. Art. No.: CD012573. DOI: 10.1002/14651858.CD012573.pub3.

Copyright ( 2019 The Authors. Cochrane Database of Systematic Reviews published by John Wiley \& Sons, Ltd. on behalf of The Cochrane Collaboration. This is an open access article under the terms of the Creative Commons Attribution-Non-Commercial Licence, which permits use, distribution and reproduction in any medium, provided the original work is properly cited and is not used for commercial purposes.

\section{A B S T R A C T}

\section{Background}

Overconsumption of food, alcohol, and tobacco products increases the risk of non-communicable diseases. Interventions to change characteristics of physical micro-environments where people may select or consume these products - including shops, restaurants, workplaces, and schools - are of considerable public health policy and research interest. This review addresses two types of intervention within such environments: altering the availability (the range and/or amount of options) of these products, or their proximity (the distance at which they are positioned) to potential consumers.

\section{Objectives}

1. To assess the impact on selection and consumption of altering the availability or proximity of (a) food (including non-alcoholic beverages), (b) alcohol, and (c) tobacco products.

2. To assess the extent to which the impact of these interventions is modified by characteristics of: i. studies, ii. interventions, and iii. participants.

\section{Search methods}

We searched CENTRAL, MEDLINE, Embase, PsycINFO, and seven other published or grey literature databases, as well as trial registries and key websites, up to 23 July 2018, followed by citation searches.

\section{Selection criteria}

We included randomised controlled trials with between-participants (parallel group) or within-participants (cross-over) designs. Eligible studies compared effects of exposure to at least two different levels of availability of a product or its proximity, and included a measure of selection or consumption of the manipulated product. 


\section{Data collection and analysis}

We used a novel semi-automated screening workflow and applied standard Cochrane methods to select eligible studies, collect data, and assess risk of bias. In separate analyses for availability interventions and proximity interventions, we combined results using randomeffects meta-analysis and meta-regression models to estimate summary effect sizes (as standardised mean differences (SMDs)) and to investigate associations between summary effect sizes and selected study, intervention, or participant characteristics. We rated the certainty of evidence for each outcome using GRADE.

\section{Main results}

We included 24 studies, with the majority (20/24) giving concerns about risk of bias. All of the included studies investigated food products; none investigated alcohol or tobacco. The majority were conducted in laboratory settings (14/24), with adult participants (17/24), and used between-participants designs (19/24). All studies were conducted in high-income countries, predominantly in the USA (14/24).

Six studies investigated availability interventions, of which two changed the absolute number of different options available, and four altered the relative proportion of less-healthy (to healthier) options. Most studies (4/6) manipulated snack foods or drinks. For selection outcomes, meta-analysis of three comparisons from three studies $(n=154)$ found that exposure to fewer options resulted in a large reduction in selection of the targeted food(s): SMD -1.13 (95\% confidence interval $(\mathrm{Cl})-1.90$ to -0.37 ) (low certainty evidence). For consumption outcomes, meta-analysis of three comparisons from two studies $(n=150)$ found that exposure to fewer options resulted in a moderate reduction in consumption of those foods, but with considerable uncertainty: SMD $-0.55(95 \% \mathrm{Cl}-1.27$ to 0.18$)$ (low certainty evidence).

Eighteen studies investigated proximity interventions. Most (14/18) changed the distance at which a snack food or drink was placed from the participants, whilst four studies changed the order of meal components encountered along a line. For selection outcomes, only one study with one comparison $(n=41)$ was identified, which found that food placed farther away resulted in a moderate reduction in its selection: SMD -0.65 ( $95 \% \mathrm{Cl}-1.29$ to -0.01 ) (very low certainty evidence). For consumption outcomes, meta-analysis of 15 comparisons from 12 studies $(n=1098)$ found that exposure to food placed farther away resulted in a moderate reduction in its consumption: SMD $-0.60(95 \% \mathrm{Cl}-0.84$ to -0.36$)$ (low certainty evidence). Meta-regression analyses indicated that this effect was greater: the farther away the product was placed; when only the targeted product(s) was available; when participants were of low deprivation status; and when the study was at high risk of bias.

\section{Authors' conclusions}

The current evidence suggests that changing the number of available food options or altering the positioning of foods could contribute to meaningful changes in behaviour, justifying policy actions to promote such changes within food environments. However, the certainty of this evidence as assessed by GRADE is low or very low. To enable more certain and generalisable conclusions about these potentially important effects, further research is warranted in real-world settings, intervening across a wider range of foods - as well as alcohol and tobacco products - and over sustained time periods.

\section{PLAIN LANGUAGE SUMMARY}

\section{Altering the availability or proximity of food, alcohol, and tobacco products to change their selection and consumption}

Unhealthy patterns of consumption of food, alcohol, and tobacco products are important causes of ill health. Changing the availability (the range or amount of options, or both) of these products or their proximity (the distance at which they are positioned) to potential consumers could help people make healthier choices.

\section{What is the aim of this review?}

This review investigated whether altering the availability or proximity of food (including non-alcoholic beverages), alcohol, and tobacco products changed people's selection (such as purchasing) or consumption of those products. We searched for all available evidence from randomised controlled trials (a type of study in which participants are assigned to one of two or more treatment groups using a random method) to answer this question, and found 24 studies, all of which were conducted in high-income countries.

\section{What are the main results of the review?}

Six studies involved availability interventions, of which four changed the relative proportion of less-healthy to healthier options, and two changed the absolute number of different options available. In statistical analyses that combined results from multiple studies, it was found that reducing the number of available options for a particular range or category of food(s) reduced selection of those food products (from analysing 154 participants) and possibly reduced consumption of those products (from 150 participants). However, the certainty of the evidence for these effects was low.

Eighteen studies involved proximity interventions. Most (14/18) changed the distance at which a snack food or drink was placed from the participants, whilst four studies changed the order of meal components encountered along a line. One study found that this reduced selection of food (from analysing 41 participants), whilst in a statistical analysis combining results from multiple studies, it was found that 
placing food farther away reduced consumption of those food products (from analysing 1098 participants). However, the certainty of the evidence for these effects was very low and low, respectively.

\section{Key messages}

Mindful of its limitations, the current evidence suggests that changing the number of available food options or changing where foods are positioned could contribute to meaningful changes in behaviour, justifying policy actions to promote such changes to food environments. However, more high-quality studies in real-world settings are needed to make this finding more certain.

\section{How up-to-date is this review?}

The evidence is current to 23 July 2018. 


\section{SUMMARY OF FINDINGS}

Summary of findings for the main comparison. Lower versus higher availability (i.e. fewer versus more options) of food products for changing quantity of food selected or consumed

\section{Lower versus higher availability of food products for changing quantity of food selected or consumed}

Population: Adults and children

Setting: Field and laboratory settings

Intervention: Lower availability of food products (fewer options)

Comparison: Higher availability of food products (more options)

\begin{tabular}{|c|c|c|c|c|c|}
\hline \multirow[t]{2}{*}{ Outcomes } & \multicolumn{2}{|c|}{ Illustrative comparative risks (95\% Cl) } & \multirow{2}{*}{$\begin{array}{l}\text { Relative effect } \\
(95 \% \mathrm{Cl})\end{array}$} & \multirow{2}{*}{$\begin{array}{l}\text { Number } \\
\text { of partici- } \\
\text { pants } \\
\text { (studies; } \\
\text { compar- } \\
\text { isons) }\end{array}$} & \multirow{2}{*}{$\begin{array}{l}\text { Certainty of } \\
\text { evidence } \\
\text { (GRADE) }\end{array}$} \\
\hline & $\begin{array}{l}\text { Assumed risk: higher avail- } \\
\text { ability of food products (more } \\
\text { options) }\end{array}$ & $\begin{array}{l}\text { Corresponding risk: lower availability of food } \\
\text { products (fewer options) }\end{array}$ & & & \\
\hline Selection & $\begin{array}{l}\text { Mean energy selected on an av- } \\
\text { erage snack occasion of } 200 \\
( \pm 63) \mathrm{kcal}^{1}\end{array}$ & $\begin{array}{l}\text { Mean energy selected on an average snack occa- } \\
\text { sion would be } 71 \mathrm{kcal}(35.6 \%) \text { less with lower avail- } \\
\text { ability ( } 120 \mathrm{kcal} \text { fewer to } 23 \mathrm{kcal} \text { fewer; } 59.9 \% \text { less } \\
\text { to } 11.7 \% \text { less). }\end{array}$ & $\begin{array}{l}\text { Mean selection in the lower avail- } \\
\text { ability group was } 1.13 \text { standard de- } \\
\text { viations lower ( } 1.90 \text { lower to } 0.37 \\
\text { lower). }\end{array}$ & $\begin{array}{l}154 \\
\text { (3 RCTs; } 3 \\
\text { compar- } \\
\text { isons) }\end{array}$ & $\begin{array}{l}\oplus \oplus \odot \odot \\
\text { LOW } 23\end{array}$ \\
\hline $\begin{array}{l}\text { Consump- } \\
\text { tion }\end{array}$ & $\begin{array}{l}\text { Mean energy intake on an aver- } \\
\text { age snack occasion of } 200( \pm 63) \\
\text { kcal }\end{array}$ & $\begin{array}{l}\text { Mean energy intake on an average snack occasion } \\
\text { would be } 35 \mathrm{kcal}(17.3 \%) \text { less with lower availabili- } \\
\text { ty ( } 80 \mathrm{kcal} \text { fewer to } 11 \mathrm{kcal} \text { more; } 40 \% \text { less to } 5.7 \% \\
\text { more). }\end{array}$ & $\begin{array}{l}\text { Mean consumption in the lower } \\
\text { availability group was } 0.55 \text { stan- } \\
\text { dard deviations lower ( } 1.27 \text { lower } \\
\text { to } 0.18 \text { more). }\end{array}$ & $\begin{array}{l}150 \\
(2 \text { RCTs; } 3 \\
\text { compar- } \\
\text { isons) }\end{array}$ & $\begin{array}{l}\oplus \oplus \Theta \odot \\
\text { LOW } 24\end{array}$ \\
\hline
\end{tabular}

The basis for the assumed risk is provided in Footnotes. 5 The corresponding risk (and its 95\% confidence interval) is based on the assumed risk and the relative effect of the intervention (and its $95 \% \mathrm{Cl}$ ). The relative effect is derived from the primary random-effects meta-analysis for the outcome.

Cl: confidence interval; kcal: kilocalories; $\mathbf{R C T}$ : randomised controlled trial

\section{GRADE Working Group grades of evidence}

High certainty: The current evidence provides a very good indication of the likely effect, and the likelihood that the actual effect will be substantially different is low. Moderate certainty: The current evidence provides a good indication of the likely effect, and the likelihood that the actual effect of the treatment will not be substantially different is moderate.

Low certainty: The current evidence provides some indication of the likely effect, but the likelihood that the actual effect will be substantially different is high.

Very low certainty: The current evidence does not provide a reliable indication of the likely effect, and the likelihood that the actual effect will be substantially different is very high.

1 Assumes that all foods selected are consumed.

- 2Downgraded by one level for study limitations: study-level estimates of this effect were judged to have significant concerns related to risk of bias. 
3Downgraded by one level for imprecision: the number of participants (effective sample size) incorporated into analysis is less than the number of participants required by a conventional sample size calculation for a single adequately powered trial (optimal information size), and confidence intervals are wide.

${ }^{4}$ Downgraded by one level for imprecision: the number of participants (effective sample size) incorporated into analysis is less than the number of participants required by a conventional sample size calculation for a single adequately powered trial (optimal information size). The confidence intervals are wide and include the possibility of a small effect on increasing consumption.

${ }^{5}$ Estimates of variance are based on data from a representative sample of UK adults, from the UK National Diet and Nutrition Survey Years 7-8 (Public Health England 2018a); see Effects of interventions for details.

Summary of findings 2. Lower versus higher proximity (i.e. placed farther away versus placed nearer) of food products for changing quantity of food selected or consumed

\section{Lower versus higher proximity of food products for changing quantity of food selected or consumed}

Patient or population: Adults and children

Setting: Field and laboratory settings

Intervention: Lower proximity of food products (placed farther away)

Comparison: Higher proximity of food products (placed nearer)

\begin{tabular}{|c|c|c|c|c|c|}
\hline \multirow[t]{2}{*}{ Outcomes } & \multicolumn{2}{|c|}{ Illustrative comparative risks ( $95 \% \mathrm{CI}$ ) } & \multirow{2}{*}{$\begin{array}{l}\text { Relative effect } \\
(95 \% \mathrm{Cl})\end{array}$} & \multirow{2}{*}{$\begin{array}{l}\text { Number } \\
\text { of partici- } \\
\text { pants } \\
\text { (studies; } \\
\text { compar- } \\
\text { isons) }\end{array}$} & \multirow{2}{*}{$\begin{array}{l}\text { Certainty of } \\
\text { evidence } \\
\text { (GRADE) }\end{array}$} \\
\hline & $\begin{array}{l}\text { Assumed risk: higher prox- } \\
\text { imity of food products } \\
\text { (placed nearer) }\end{array}$ & $\begin{array}{l}\text { Corresponding risk: lower proximity of food prod- } \\
\text { ucts (placed farther away) }\end{array}$ & & & \\
\hline Selection & $\begin{array}{l}\text { Mean energy selected on an } \\
\text { average snack occasion of } \\
200( \pm 63) \text { kcal }^{1}\end{array}$ & $\begin{array}{l}\text { Mean energy selected on an average snack occasion } \\
\text { would be } 41 \mathrm{kcal}(20.5 \%) \text { less with lower proximi- } \\
\text { ty ( } 81 \mathrm{kcal} \text { fewer to } 1 \mathrm{kcal} \text { fewer; } 40.6 \% \text { less to } 0.3 \% \\
\text { less). }\end{array}$ & $\begin{array}{l}\text { Mean selection in the lower prox- } \\
\text { imity group was } 0.65 \text { standard de- } \\
\text { viations lower ( } 1.29 \text { lower to } 0.01 \\
\text { lower). }\end{array}$ & $\begin{array}{l}41 \text { ( } 1 \text { RCT; } \\
1 \text { compari- } \\
\text { son) }\end{array}$ & $\begin{array}{l}\oplus \ominus \ominus \ominus \\
\text { VERY LOW } 2 \\
34\end{array}$ \\
\hline $\begin{array}{l}\text { Consump- } \\
\text { tion }\end{array}$ & $\begin{array}{l}\text { Mean energy intake on an av- } \\
\text { erage snack occasion of } 200 \\
( \pm 63) \text { kcal }\end{array}$ & $\begin{array}{l}\text { Mean energy intake on an average snack occasion } \\
\text { would be } 38 \mathrm{kcal}(18.9 \%) \text { less with lower proximity } \\
\text { ( } 53 \mathrm{kcal} \text { fewer to } 23 \mathrm{kcal} \text { fewer; } 26.5 \% \text { less to } 11.3 \% \\
\text { less). }\end{array}$ & $\begin{array}{l}\text { Mean consumption in the lower } \\
\text { availability group was } 0.60 \text { stan- } \\
\text { dard deviations lower ( } 0.84 \text { lower } \\
\text { to } 0.36 \text { lower). }\end{array}$ & $\begin{array}{l}1098(12 \\
\text { RCTs; } 15 \\
\text { compar- } \\
\text { isons) }\end{array}$ & $\begin{array}{l}\oplus \oplus \odot \odot \\
\text { LOW } 25\end{array}$ \\
\hline
\end{tabular}

The basis for the assumed risk is provided in Footnotes. 6 The corresponding risk (and its $95 \%$ confidence interval) is based on the assumed risk and the relative effect of the intervention (and its $95 \% \mathrm{Cl}$ ). The relative effect is derived from the primary random-effects meta-analysis for the outcome.

Cl: confidence interval; kcal: kilocalories; $\mathbf{R C T}$ : randomised controlled trial

\section{GRADE Working Group grades of evidence}

High certainty: The current evidence provides a very good indication of the likely effect, and the likelihood that the actual effect will be substantially different is low. Moderate certainty: The current evidence provides a good indication of the likely effect, and the likelihood that the actual effect of the treatment will not be substantially different is moderate. 
Low certainty: The current evidence provides some indication of the likely effect, but the likelihood that the actual effect will be substantially different is high.

Very low certainty: The current evidence does not provide a reliable indication of the likely effect, and the likelihood that the actual effect will be substantially different is very high.

\section{Assumes that all foods selected are consumed.}

2Downgraded by one level for study limitations: study-level estimates of this effect were judged to have significant concerns related to risk of bias.

3Downgraded by one level for imprecision: the effect estimate derives from a single small study.

${ }^{4}$ Downgraded by one level for indirectness: all data derived from a study conducted in a laboratory setting, meaning it may be less directly informative to real-world implementation of the intervention.

${ }^{5}$ Downgraded by one level for publication bias: formal assessment of the degree of asymmetry present in a funnel plot suggested the presence of publication bias.

${ }^{6}$ Estimates of variance are based on data from a representative sample of UK adults, from the UK National Diet and Nutrition Survey Years 7-8 (Public Health England 2018a); see Effects of interventions for details. 


\section{B A C K G R O U N D}

\section{Description of the condition}

Non-communicable diseases, principally cardiovascular diseases, diabetes, certain forms of cancer, and chronic respiratory diseases, accounted for an estimated $68 \%$ of all deaths worldwide in 2012 (WHO 2016). Major risk factors for non-communicable diseases include metabolic and dietary risk factors linked to food consumption (e.g. high body mass index, high systolic blood pressure), as well as smoking and alcohol use - risks that are, in principle, modifiable. These are also amongst the most significant risk factors for total disease burden, both globally and in highincome countries specifically (GBD 2018). Identifying interventions that are effective in achieving sustained health behaviour change across populations and countries is therefore one of the most important public health challenges of the 21st century.

\section{Description of the intervention}

It is increasingly recognised that the physical environments that surround us can exert considerable influences on our health-related behaviours and that altering these environments may provide a catalyst for behaviour change (Cohen 2016; Marteau 2012; Stok 2017). We have previously described a set of interventions that involve altering small-scale physical environments - or micro-environments - with the intention of changing health-related behaviours (Hollands 2013a; Hollands 2017a), which have also been described as 'choice architecture' (or 'nudge') interventions (Cadario in press; Szaszi 2018; Thaler 2008). These interventions involve changing characteristics of, or cues within, environments where people may select or consume food, alcohol, or tobacco including restaurants, workplaces, schools, homes, bars, pubs, supermarkets, or shops. They have received increased policy and research interest in recent years as a result of several factors (Marteau 2015), including shifts in theoretical understanding, supportive empirical evidence, political acceptability (with governments preferring 'light-touch' rather than legislative or regulatory approaches), and public acceptability (with evidence suggesting these types of interventions are relatively acceptable) (Petrescu 2016; Reisch 2016; Reynolds 2019). Perceived feasibility and low cost, whereby such interventions may be viewed as easily implemented at scale without complex legislative or regulatory processes or the need for individual delivery, may also contribute.

The placement of food, alcohol, and tobacco products within the physical environment can influence their selection and consumption. Within the Typology of Interventions in Proximal Physical Micro-Environments (TIPPME) intervention typology (Hollands 2017a), a framework developed for characterising interventions in physical micro-environments, 'placement' interventions comprise two key, more specific intervention types: first, interventions that target the 'availability' of food, alcohol, or tobacco products within a specific environment - essentially, what is made available for selection or consumption, or both; and second, interventions focused on how available products are positioned within a specific environment. Our specific focus with respect to how products are positioned is on the 'proximity' of food, alcohol, or tobacco products to and from people, which can be altered by moving the products nearer or farther away to make them more or less accessible. Availability and proximity interventions are described further below.

\section{Interventions that alter availability}

These interventions involve manipulating the available food, alcohol, or tobacco product options in an environment such as a shop, bar, or restaurant. This can be achieved by providing, either:

a) a greater or lesser range of different product options (within a targeted range or category), for example:

- food - providing a wider range of healthier meal options, or a reduced number of less-healthy meal options in a restaurant or cafeteria; or a reduced range of snacks in vending machines;

- alcohol - providing a wider range of different low-alcohol options in a bar or pub; or a reduced range of types of wine or beer in a restaurant; and

- tobacco - providing a reduced range of types of tobacco product in a shop.

b) a greater or lesser amount (number) of discrete units of a product. In this case, the range of different product options might not be changed, but the number of available units of the existing product options is manipulated. For example:

- food - making a lesser amount of (a range of) chocolate bars on display in a supermarket;

- alcohol - making a greater amount of (a range of) low-alcohol beer bottles available in a bar or pub; and

- tobacco - making a lesser amount of (a range of) cigarettes available in a shop.

c) a combination of $a$ ) and b).

These possible manipulations can concern changes in the absolute number of different options available, or changes in relative proportions, such as the relative number (proportion) of lesshealthy (to healthier) options that are available.

\section{Interventions that alter proximity}

These interventions concern the positioning of products that are available within that environment. The term we have used 'proximity' - reflects the fact that the predominant intervention of this type within the current context involves moving food, alcohol, or tobacco products closer to or farther away from people, such as placing a healthier product such as fruit in a more proximal (and therefore convenient) position within a shop to encourage its purchase (Kroese 2016). By reducing or increasing the distance to be traversed or reached, such interventions can alter the degree of convenience, and of effort required for potential consumers to select or consume these products.

The proximity of a product (how close or far away it is) is altered in relation to key physical features in environments, such as typical or expected walking routes, building entrances, checkouts in supermarkets or shops, or seating. Examples include positioning a display of food products close to a shop's entrance (e.g. $1 \mathrm{~m}$ ), aiming to enable convenient selection of the products, versus this being located at a distance that requires customers to walk a greater distance to engage with the display (e.g. 20 m). Alternatively, it could involve altering the positioning of a food product to be within arm's reach of a potential consumer (e.g. placed $20 \mathrm{~cm}$ from seating) versus requiring them to leave their seating and walk to take the food product (e.g. placed $2 \mathrm{~m}$ from seating). 
A detailed conceptual framework for these interventions has been developed (Pechey under review).

\section{How the intervention might work}

There are considerable influences on behaviour that are beyond individuals' deliberative control. Indeed, it has been suggested that much human behaviour occurs outside of awareness, cued by stimuli in environments and resulting in actions that may be largely unaccompanied by conscious reflection (Marteau 2012; Neal 2006). This proposition has led to increasing policy and research attention being placed on interventions with mechanisms of action that may be less dependent on the conscious engagement of the recipients (Hollands 2016), including interventions that involve altering the placement of objects within the physical environments that surround and cue behaviour.

Various underlying mechanisms of action have been proposed for both availability and proximity interventions (Pechey under review), although it is difficult to assess these outside of artificial, controlled environments. In relation to availability, whether options are available (or absent) within a given environment inevitably shapes and constrains people's possible responses. The more product options that are available, the more likely it is that an actor will encounter an option they are willing to select or consume (Chernev 2011). Exposure may also increase the salience of, and the attention directed towards, products and elicit a 'mere-exposure' effect - whereby repeated exposure to a product can elicit increased liking (Dalenberg 2014). Altering a range of available products could also have the effect of implying a new social norm about which types of products are acceptable or commonplace, and this could influence selection and consumption. Whilst currently largely unexplored, the sum of these potential mechanisms is that increasing the range of options for a given product or category should increase its selection or consumption, albeit subject to people engaging with the product in the first place. This will be influenced by many factors, including characteristics of the person (such as hunger) and of the product (such as its attractiveness or palatability). In addition, it has been suggested that if the range of available products is increased, choosing between these options becomes more reliant on a reasoning process, meaning that people may make different choices based on what they are most able to justify (Sela 2009). Furthermore, if the range of available product options remains the same, but the number of units of these products increases, this may increase their visibility or salience and therefore encourage selection or consumption.

In relation to proximity, the central role of physical and mental effort has been highlighted (Bar-Hillel 2015). Humans tend to take the least effortful course of action without the need for conscious deliberation, and so physical environments can shape responses by capitalising on this phenomenon. Consequently, products placed nearer an actor require less effort to obtain than those placed farther away, and this may correspondingly impact on motivation to select or consume them (Hunter 2018). Other than the effort needed (or perceived as such), more distal products may also be less visible and less salient (Maas 2012). Increasing physical distance may also increase 'psychological distance' - the subjective experience of distance from the self in that time and place - and so more distal products may be focused upon in a less detailed way or be subject to more deliberation or rationalisation, which may impact one's behaviour (Trope 2010).

\section{Why it is important to do this review}

A systematic scoping review of evidence for the effects of physical micro-environment interventions identified a substantial number of studies that have investigated the effects of altering the availability and proximity of products on health-related behaviours (Hollands 2013b). The majority of these studies focused on food products, where interventions have significant potential given the necessity of consumption of these products and their ubiquity within many environments. However, because both tobacco and alcohol use also involve the selection and consumption of products, such interventions may also have the potential to change these behaviours via similar mechanisms. We have synthesised evidence for the effects of availability and proximity interventions within a single systematic review because we conceptualise them both as interventions that alter the placement of products within physical micro-environments. To our knowledge, evidence from these studies has yet to be synthesised using rigorous systematic review methods that include quantitative synthesis, assessment of risk of bias, and investigation of potential effect modifiers, or to encompass alcohol and tobacco use, although parts of this evidence base have been reviewed. As such, we do not yet have reliable estimates of the effects of these types of interventions on product selection and consumption, nor of the influence of factors that may modify any such effects. Both are necessary to inform the selection and design of effective public health interventions, particularly given increasing research and policy interest in interventions that alter the physical environment to make unhealthier behaviours less likely and healthier behaviours more likely. This interest is evidenced by the substantial public and policy interest in a previous Cochrane Review on portion, package, and tableware size (Hollands 2015), which has influenced policy debate in the UK and Australia (Jones 2016).

Poor diet, harmful alcohol use, and smoking are socially patterned, being more common amongst those in lower socioeconomic positions, thereby contributing to the increased morbidity and premature mortality observed in these groups (Stringhini 2010). Behaviour change interventions that focus on the provision of educational information to individuals and encouragement for them to make active choices, potentially widen health inequalities (Lorenc 2013; McGill 2015). Interventions that instead aim to alter the environments that people are exposed to and therefore may be less reliant on conscious, reflective engagement (Hollands 2016), could have a greater potential to reduce, or at least not increase, health inequalities. It has been suggested that this may be because they rely less on recipients' cognitive resources including levels of literacy, numeracy, and cognitive control, which on average are lower in population subgroups experiencing higher levels of social and material deprivation (Hall 2014). The current review sought to identify evidence for differential effects of exposure to these interventions between socioeconomic groups. To our knowledge, no studies of the effects of these interventions have been conducted in low- and middle-income countries (LMICs) that would enable a comparison of effects between studies in highincome countries (HICS) and LMICs, but we sought to identify such evidence. Purposively considering socioeconomic status and country context factors in our analysis (and highlighting gaps in the evidence base) enabled the opportunity to assess the potential impact such interventions could have upon health inequalities. 


\section{O B JECTIVES}

1. To assess the impact on selection and consumption of altering the availability or proximity of (a) food (including non-alcoholic beverages), (b) alcohol, and (c) tobacco products.

2. To assess the extent to which the impact of these interventions is modified by characteristics of: i. studies, ii. interventions, and iii. participants.

\section{METHODS}

\section{Criteria for considering studies for this review}

\section{Types of studies}

Randomised controlled trials (RCTs) or cluster-RCTs with betweenparticipants (parallel group) or within-participants (cross-over) designs, conducted in laboratory or field ('real-world') settings. We excluded non-randomised studies because, first, a scoping review indicated that a sufficient number of eligible randomised studies were likely available to enable quantitative synthesis of evidence for intervention effects (Hollands 2013a). Second, compared with RCTs, non-randomised studies rely on more stringent and sometimes non-verifiable assumptions in order to confer confidence that the risk of systematic differences between comparison groups beyond the intervention of interest (i.e. confounding) is sufficiently low to permit valid inferences about causal effects. If randomised assignment was not clear in studies otherwise considered eligible for inclusion at the full-text assessment stage, we only included the study if study authors had confirmed that randomisation occurred. We also excluded randomised studies that had only a single participating site in the intervention or the comparator group, or both, because this would result in the treatment effect being completely confounded with the site characteristics.

\section{Types of participants}

Adults and children exposed to the interventions. We defined adults as those 18 years of age or over, and children as those under 18 years (United Nations 1989). We excluded studies where the product was selected and fed directly by one person to another (e.g. mother-child dyads). No other exclusion criteria in relation to demographic, socioeconomic, or clinical characteristics were set. We excluded studies involving non-human participants (i.e. animal studies).

\section{Types of interventions}

Eligible interventions were those that involved altering the availability or proximity of food (including non-alcoholic beverages), alcohol, or tobacco products within 'physical microenvironments', defined here as small-scale physical environments where people gather for specific purposes and activities, such as restaurants, workplaces, schools, homes, bars, pubs, supermarkets, or shops (Hollands 2017a; Swinburn 1999). Availability interventions and proximity interventions are defined in the Description of the intervention section, and details of specific eligibility criteria for each intervention type are provided below.

\section{Availability interventions}

'Availability interventions' eligible for consideration in this review were those that involved comparing the effects of exposure to at least two differing (i.e. higher versus lower) levels of availability of a manipulated food, alcohol, or tobacco product. This allowed us to examine whether, for example, making a food product more available increases its consumption, or making a food product less available decreases its consumption. The 'product' can be operationalised as applying to types of a specific product (e.g. fruit, chocolate bars) or to broader ranges or categories of products (e.g. energy-dense snack foods; low-fat meals). For alcohol and tobacco products, we also considered including interventions in which the availability of specific recognised alternatives to those products that are not themselves alcohol and tobacco products is manipulated within alcohol or tobacco selection and consumption contexts (e.g. alcohol-free variants in the case of alcohol, or ecigarettes in the case of tobacco).

\section{Additional inclusion criteria}

1. The comparison of different levels of availability must be explicitly described, as opposed to this being inferred by the review team. For example, a review author could infer that a supermarket sales promotion would increase the number of products on display in store, but a study would only be included if this was clearly stated by the authors.

2. We included multicomponent interventions in which there were concurrent intervention components that were unrelated to availability, providing those additional components were implemented wholly within the same physical microenvironment as in the availability intervention, involving changes to the product itself or its proximal physical environment. Examples include nutritional labelling on the product itself, or promotional signage placed near to the product. We planned to treat confounded and unconfounded components differently (see Data synthesis).

We excluded the following interventions.

1. Multicomponent interventions in which there were concurrent intervention components that were unrelated to availability, where those additional components were not implemented wholly within the same micro-environment as in the availability intervention, involving changes to the product itself or its proximal physical environment. Examples of such ineligible intervention components include health education programmes or marketing campaigns.

2. Interventions in which availability may be altered indirectly as a result of a higher-level intervention but is not directly and systematically altered (e.g. organisational-level interventions to encourage the wider availability of healthier products within a workplace or set of workplaces, or national- or regionallevel policy interventions to encourage schools to modify their environments). Whilst availability may be changed as a result of the higher-level intervention, this is not directly manipulated to safeguard implementation fidelity.

3. Interventions within analogue studies that do not manipulate real food, alcohol, or tobacco products but instead may use written vignettes, computer or questionnaire tasks, or mock products to assess the impact of altering availability.

4. Interventions in which the range of product options is unchanged (as regards being perceptible prior to selection) in terms of the different types or categories of products that are available, but changes are made in the range of ways in which those same products are formulated (as regards being 
perceptible prior to selection) or presented, such as flavour, colour, size, or shape.

5. Interventions in which the environmental contexts or opportunities for selection and consumption are not comparable between intervention and control groups. We therefore excluded interventions that involved removing (or adding) the entire range of food, alcohol, or tobacco products within a given micro-environment (e.g. studies examining the effectiveness within a specified environment of complete smoking or alcohol bans), as well as those that involved substantial changes to its infrastructure (such as building new shops or restaurants) or its furniture (e.g. adding or removing fixtures and fittings). We also excluded interventions in which availability differed between intervention and control arms due to: additional exposure to foods via assigned dietary programmes (e.g. prescribed diets); education (e.g. taste-testing sessions, cooking lessons, or food education); or other means of prescribed distribution of products to participants.

6. Interventions in which the availability of a product was not altered in terms of its range or amount but as a result of temporal (e.g. changing hours of sale or altering a range of available products over time) or spatial (e.g. changing the places in which a product can be selected or consumed) factors (Han 2014; Sherk 2018).

\section{Proximity interventions}

'Proximity interventions' eligible for consideration in this review were those that involved comparing the effects of exposure to at least two differing (i.e. higher versus lower) levels of proximity of a manipulated food, alcohol, or tobacco product. Whilst there may be other ways of altering the positioning of products that do not impact on their proximity, we have purposefully limited our scope to proximity interventions. This is because any other such studies would be difficult to assess within the same framework specified for use in the current review, which focuses on the effects of altering the quantity or degree (i.e. increase versus decrease) of a specific property (i.e. proximity)

\section{Additional inclusion criteria}

1. The comparison of different levels of proximity had to be explicitly described, as opposed to this being inferred by the review team. For example, a review author could infer that a redesigned layout of a cafeteria or restaurant might increase or decrease proximity from a given point of reference, but a study would only be included if this change in proximity was clearly stated by authors.

2. As per availability interventions, we included multicomponent interventions in which there were concurrent intervention components that were unrelated to proximity, providing those additional components were implemented wholly within the same physical micro-environment as in the proximity intervention, involving changes to the product itself or its proximal physical environment. Examples include nutritional labelling on the product itself, or promotional signage placed near to the product.

We excluded the following interventions.

1. As per availability interventions, multicomponent interventions in which there were concurrent intervention components that were unrelated to proximity, where those additional components were not implemented wholly within the same micro-environment as in the proximity intervention, involving changes to the product itself or its proximal physical environment.

2. Interventions in which proximity may be altered indirectly as a result of a higher-level intervention but was not directly and systematically altered (e.g. organisational-level interventions to encourage the redesign of the layout of school or workplace cafeterias). Whilst proximity may be changed as a result of the higher-level intervention, this is not directly manipulated to safeguard implementation fidelity.

3. Interventions within analogue studies that do not manipulate real food, alcohol, or tobacco products but instead may use written vignettes, computer or questionnaire tasks, or mock products to assess the impact of altering proximity.

4. Interventions in which the proximity of text, symbols, or images that relate to products is altered (e.g. on a sign, advertisement, poster, menu, leaflet, or computer screen (e.g. online supermarket)), but the proximity of the actual products to be selected or consumed is not.

5. Interventions in which the environmental contexts or opportunities for selection and consumption are not comparable between intervention and control groups. We therefore excluded interventions that involved substantial changes to the infrastructure of the environment or its furniture.

Studies including both availability and proximity intervention components were eligible for inclusion in the review.

\section{Types of outcome measures}

Eligible studies had to incorporate one or more objective measures of unconstrained selection (with or without purchasing) or consumption of the manipulated food, alcohol, or tobacco product(s). For example, a study investigating the effects of increasing the availability or proximity of fruit within a shop on healthier purchasing could include a specific measure of fruit (i.e. the manipulated product) selected only, or a broader measure at category level that encompasses both fruit selection and selection of non-fruit options available in the shop (e.g. a measure of selection of all healthier food options). Either would represent an appropriate primary outcome. Studies may additionally include measures that relate specifically to non-manipulated products in the given example there may also be a measure of selection of non-fruit options only. Such measures would represent appropriate secondary outcomes.

Objective measurement may involve sales data or calculating the amount of a product consumed by subtracting the amount remaining after consumption from the total amount presented to the participant. Alternatively, it may involve direct observation of selection or consumption behaviour by outcome assessors. Subjective measurement would involve participant self-report. By unconstrained, we refer to behaviour of participants that is not constrained or regulated by either explicit instructions or some other action of the researcher. For example, we excluded studies that manipulated the availability of foods that are not selected, plated, or served under the direction of the participant, but where foods were presented to them individually with the instruction to select or consume. 
Quantities selected or consumed may have been measured over a time period less than or equal to one day (immediate) or exceeding one day (longer term). Our choice of eligible outcome constructs reflects a focus on the assessment of the effects of eligible interventions in terms of the types and amounts of food, alcohol, and tobacco people consume, coupled with recognition that the amount selected (with or without purchasing) is an important intermediate endpoint in pathways to consumption.

\section{Primary outcomes}

Measures of unconstrained selection (with or without purchasing) or consumption of the manipulated food, alcohol, or tobacco product(s). We anticipated encountering a range of measures of these outcome constructs amongst included studies, and present the following examples of likely measures below.

1. Selection of a product (a) without purchase, or b) with purchase.

Assessment of the amounts of products (e.g. food, drink, alcohol, or tobacco products), energy or substances (e.g. saturated fat, alcohol, carbon monoxide) selected, measured in applicable natural units (e.g. kilojoules, grams). Depending on the study setting, a product may be selected with or without this involving a purchase, that is a transfer of money to the vendor. In cases where there is no purchasing, selection may be comparable to typical purchasing (e.g. products being selected in a restaurant or bar where there is no charge for them) or it may be behaviour that necessarily precedes consumption in that context, such as serving an amount of a food product onto a plate or pouring an amount of drink into a glass.

\section{Consumption (intake) of a product.}

As per selection, assessment of the amounts of products (e.g. food, drink, alcohol, or tobacco products), energy, or substances (e.g. saturated fat, alcohol, carbon monoxide) consumed, measured in applicable natural units (e.g. kilojoules, grams).

\section{Secondary outcomes}

As with the specified primary outcomes, secondary outcomes are also measures of unconstrained selection (with or without purchasing) or consumption of food, alcohol, or tobacco products. However, secondary outcomes apply to other products that are available in the same micro-environment at the same point of selection or consumption as the manipulated product(s), but that are not themselves manipulated as regards to their availability or proximity.

Due to the nature of the interventions, we anticipated that adverse effects (other than unwanted health-harming effects on selection or consumption, which would be captured by the specified primary and secondary outcomes) were unlikely to occur, be assessed or reported. However, any adverse events or harms reported in the included studies were noted.

\section{Conceptual model}

To supplement study eligibility criteria, we developed a provisional conceptual model that was published in the protocol for this review (Hollands 2017b). The conceptual model was design-oriented in the sense that it was intended to help direct the review process by providing a simplified visual representation of the causal system of interest (Anderson 2011), that is the proposed causal pathway between eligible interventions and their outcomes (behavioural endpoints), and potential moderators of that relationship (effect modifiers) given that differential effects are plausible (Anderson 2013). We used the provisional conceptual model to inform the development of search strategies, data extraction forms, and a provisional framework for the statistical analysis of the data collected from the eligible studies (see Search methods for identification of studies and Data collection and analysis).

We revised the conceptual model iteratively, as we encountered evidence from eligible studies during the course of the review process, and documented revisions. We used the iterations of the conceptual model as a reference point for the design (in the protocol), conduct, and reporting (postprotocol) of the systematic review (Anderson 2013). In practice, iterative refinement of the conceptual model involved incorporating further potential effect modifiers identified during the data collection process, which were then considered in the analysis and reporting of these data. The final version of the conceptual model is shown in Figure 1, with details of its development in Data collection and analysis. 
Figure 1. Final conceptual model. Changes from the provisional conceptual model (Hollands 2017b), comprising two additions, are shown in red type.

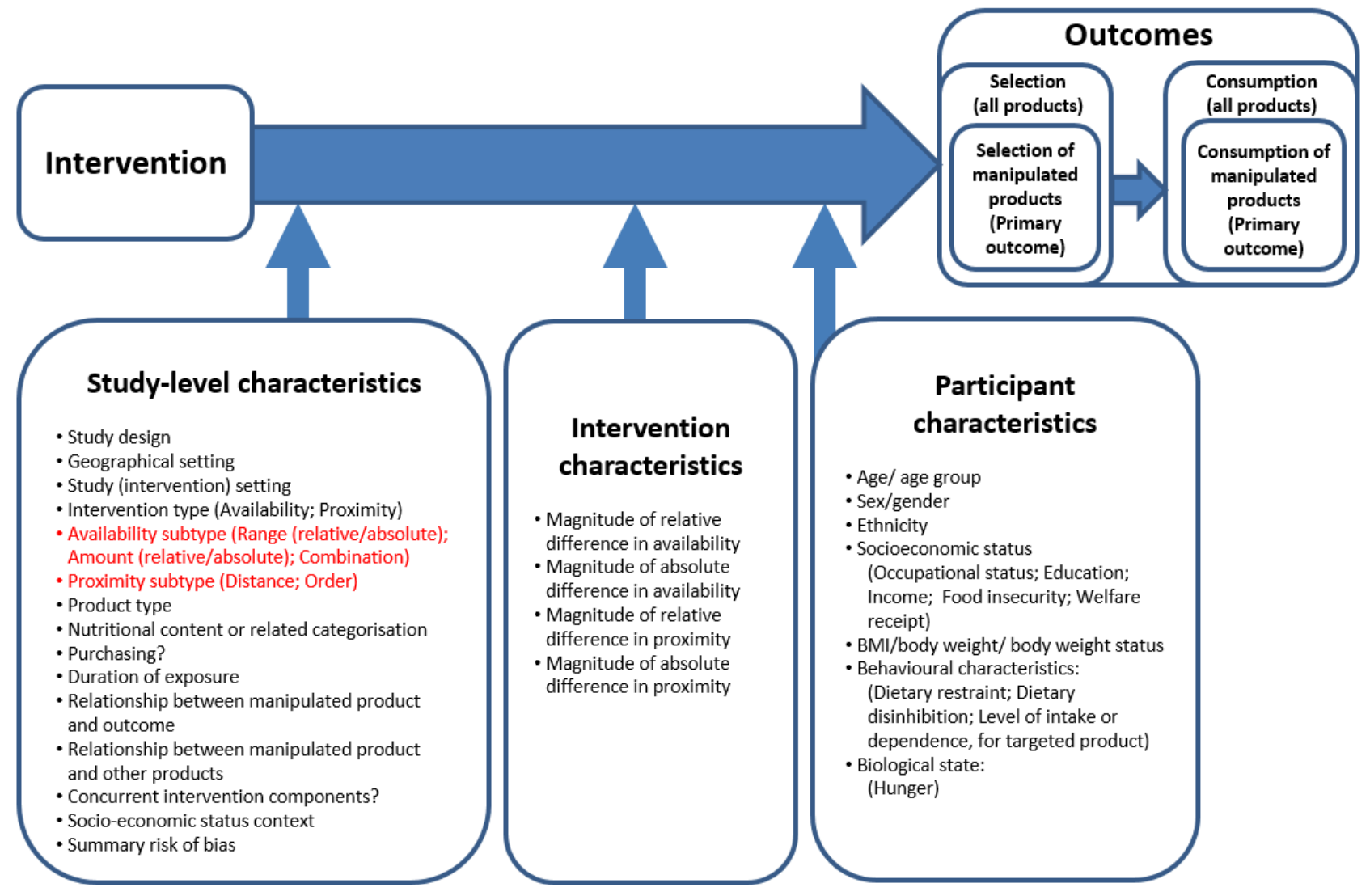

\section{Search methods for identification of studies}

\section{Electronic searches}

We developed a MEDLINE search strategy by combining sets of controlled vocabulary and free-text search terms based on the eligibility criteria described above (see Criteria for considering studies for this review). It was developed with the intention of being highly sensitive (at the expense of precision) to give confidence in its ability to detect potentially eligible title and abstract records. This search strategy was externally peer-reviewed by an information retrieval specialist and co-convenor of the Cochrane Information Retrieval Methods Group and revised based on their peer-review comments. We tested and calibrated the MEDLINE search strategy for its sensitivity to retrieve a reference set of 24 records of reports of potentially eligible studies that were identified within a preceding, broader scoping review of interventions within physical micro-environments (Hollands 2013a). The search strategy was then reviewed by the Information Specialist of the Cochrane Public Health Group and revised further based on their comments. We adapted our final MEDLINE search strategy for use in searching the other databases listed based on close examination of the database thesauri and scope notes. There were no restrictions on publication date, publication format, or language. No study design filters were incorporated. The full details of the final search strategies are provided in Appendix 1.
We conducted electronic searches for eligible studies within each of the following databases:

- Cochrane Central Register of Controlled Trials (CENTRAL) (1992 to 23rd July 2018);

- MEDLINE (including MEDLINE In-Process) (OvidSP) (1946 to 23rd July 2018);

- Embase (OvidSP) (1980 to 23rd July 2018);

- PsycINFO (OvidSP) (1806 to 23rd July 2018);

- Applied Social Sciences Index and Abstracts (ASSIA) (ProQuest) (1987 to 24th July 2018);

- Science Citation Index Expanded (Web of Science) (1900 to 24th July 2018);

- Social Sciences Citation Index (Web of Science) (1956 to 24th July 2018); and

- Trials Register of Promoting Health Interventions (EPPI Centre) (2004 to 27th July 2018).

\section{Searching other resources}

We conducted electronic searches of the following grey literature databases using search strategies adapted from the final MEDLINE search strategy, as described above:

- Conference Proceedings Citation Index - Science (Web of Science) (1990 to 24th July 2018); 
- Conference Proceedings Citation Index - Social Science \& Humanities (Web of Science) (1990 to 24th July 2018); and

- OpenGrey (1997 to 24th July 2018).

We searched trial registers (US National Institutes of Health Ongoing Trials Register ClinicalTrials.gov (www.clinicaltrials.gov/), the World Health Organization International Clinical Trials Registry Platform (apps.who.int/trialsearch/), and the EU Clinical Trials Register (www.clinicaltrialsregister.eu/)) to identify registered trials (up to 25th July 2018), and the websites of key organisations in the area of health and nutrition, including the following:

- UK Department of Health;

- Centers for Disease Control and Prevention (CDC), USA;

- World Health Organization (WHO);

- International Obesity Task Force; and

- EU Platform for Action on Diet, Physical Activity and Health.

In addition, we searched the reference lists of all eligible study reports and undertook forward citation tracking (using Google Scholar) to identify further eligible studies or study reports (up to 25th July 2018). When we found non-English language articles, we used Google Translate in the first instance to determine potential eligibility. We intended that if an article appeared to be eligible, we would have the article translated by a native language speaker or professional translation service, however no articles needed translating.

\section{Data collection and analysis}

\section{Selection of studies}

Title and abstract records retrieved by the electronic searches were imported into EPPI Reviewer 4 (ER4) systematic review software (Thomas 2010). Duplicate records were identified, reviewed manually, and removed using ER4's automatic de-duplication feature.

In relation to the electronic searches, search terms based on relevant intervention and comparator concepts (e.g. availab\$, increas\$, add\$, introduc\$, close $\$$, near $\$$, far\$) are unlikely to be specific to title-abstract records of eligible studies (even when configured in multistrand search strategies), and are also likely to feature frequently in irrelevant title-abstract records. This is likely to result in large numbers of records being retrieved by electronic searches, which need to have sufficient sensitivity to capture all eligible studies. To address this challenge, we developed a semi-automated screening workflow to manage the title-abstract screening stage, deployed in ER4, which uses machine learning to assign title-abstract records for duplicate manual screening (O'Mara-Eves 2015). This workflow was designed to maximise recall of eligible studies while reducing screening workload to match the available resource, which we expected to allow for duplicate manual screening of up to a maximum of one-third of retrieved records (the 'overall screening budget'). Further details of the semiautomated screening workflow are provided in Appendix 2.

Two review authors independently undertook duplicate screening of title and abstract records retrieved by the electronic searches. We coded title and abstract records as 'provisionally eligible', 'excluded', or 'duplicate' by applying the eligibility criteria described above (see Criteria for considering studies for this review). Any disagreements in the coding of title and abstract records were identified and resolved by discussion to reach a consensus between the two review authors. When they were unable to reach a consensus, a third review author acted as an arbiter.

We obtained full-text copies of corresponding study reports for all records coded as 'provisionally eligible' at the title and abstract screening stage. Two review authors independently undertook duplicate screening of full-text study reports, coding them as 'eligible' or 'excluded' by applying the eligibility criteria described above (see Criteria for considering studies for this review), with reasons for exclusion recorded. Any disagreements in the coding of full-text study reports or reasons for exclusion were identified and resolved by discussion to reach consensus between the two review authors. In the event that any coding disagreements could not be resolved, a third review author acted as an arbiter. Bibliographic details of study reports excluded at the full-text screening stage are provided, along with the primary reason for exclusion, in the Characteristics of excluded studies table. If we identified multiple full-text reports of the same study, we linked and treated them as a single study. Some full-text reports comprising multiple eligible studies were identified, and each study was treated separately. We documented the flow of records and studies through the systematic review process and have reported this using a PRISMA flow diagram (Moher 2009).

\section{Data extraction and management}

An electronic data extraction form was developed based on the Cochrane Public Health template and the form used in a previous Cochrane Review (Hollands 2015), modified to allow extraction of all data required for this review. An initial draft of this form was piloted using a selection of included studies, to ensure that it enabled reliable and accurate extraction of appropriate data, and was amended in consultation with the review team. One review author extracted data pertaining to the characteristics of included studies. Two review authors independently extracted outcome data in duplicate. When a study with more than two intervention arms was included, only outcome data pertaining to the intervention and comparison groups that met the eligibility criteria described above were included in the review, but the Characteristics of included studies table includes details of all intervention and comparison groups present in the study. Any discrepancies in extracted outcome data were identified and resolved by checking against the study report, and by discussion and consensus, with a third review author acting as an arbiter if necessary. We contacted study authors for key unpublished data that were missing from reports of included studies.

We collected the data summarised below, comprising 28 constructs. The 26 constructs in plain type represent the maximum core dataset that at the outset we anticipated would be required based on our study eligibility criteria and the design-oriented conceptual model. It was intended that this dataset would evolve as necessary through the review process, corresponding with revisions made to the conceptual model (see Types of outcome measures), resulting in the inclusion of two additional study characteristics in italicised text. These concerned basic subtype categorisations of availability and proximity interventions, reflecting that in the review protocol, Hollands 2017b, we had presented possible subtype categorisations that would be subject to iteration or confirmation as a result of the review process. Such 
categorisations may be subject to further elaboration in future as the empirical or theoretical basis develops (Pechey under review).

\section{Study characteristics}

1. Study design: between-participants or within-participants design; individually or cluster-randomised

2. Geographical setting: country

3. Study (intervention) setting: laboratory; field

4. Intervention type: availability; proximity

5. Availability subtype: range of different options (relative/ absolute); amount of product units (relative/absolute); combination

6. Proximity subtype: distance from set point; order encountered along line

7. Product type: food; alcohol; tobacco

8. If applicable, energy (calorie) or macronutrient content of product, and/or related categorisation (healthier versus less healthy versus mixed)

9. If applicable, selection with purchasing or selection without purchasing

10.Duration of exposure

11. Relationship between manipulated product and outcome (how outcome maps onto manipulated product)

12. Relationship between manipulated product and other available products

13. Concurrent intervention component in factorial design

14.Concurrent intervention components confounded with comparison of interest

15.Socioeconomic status context

16.Summary 'Risk of bias' assessments

17.Information on funding source and potential conflicts of interest from funding

\section{Intervention characteristics}

1. Magnitude of relative difference in availability (range, amount)

2. Magnitude of absolute difference in availability (range, amount)

3. Magnitude of relative difference in proximity

4. Magnitude of absolute difference in proximity

\section{Participant characteristics}

1. Age/age group

2. Sex/gender (e.g. male, female)

3. Ethnicity

4. Socioeconomic status (e.g. occupational status; education; income; food insecurity; welfare receipt)

5. Body mass index (BMI); body weight; body weight status

6. Behavioural characteristics (e.g. dietary restraint; dietary disinhibition; level of intake or dependence, for targeted product)

7. Biological state (e.g. hunger)

These participant characteristics cover several categories of social differentiation relevant to health equity. Collecting study-level data on these participant characteristics enabled the potential to draw inferences within our analysis concerning any differential effects of the intervention on health equity (Welch 2012). For example, proxy measures of socioeconomic status function as participant characteristics that may moderate the observed effects of the intervention on product selection and consumption. In addition, to complement investigations based on participant characteristics, we constructed a binary study-level covariate of 'socioeconomic status context' based on authors' explicit descriptions of the study sample and/or setting (see 'Study characteristics' above) that served as a proxy for the overall study context in terms of baseline levels of social and material deprivation amongst study participants. Analysis of this study-level covariate as a potential effect modifier enabled the potential to investigate specifically whether eligible interventions were more or less effective in a study context characterised by high versus low levels of social and material deprivation.

\section{Outcome data}

We anticipated that some eligible primary studies would include more than one eligible measure of selection or consumption. We used the measure of selection or consumption that mapped most closely onto the focus of the intervention, for example where only fruit products were manipulated, we used a measure that related specifically to fruit selection or consumption only. Where multiple products were manipulated concurrently, we used a measure that either related specifically to one of those products (if it was discernible that that product was the primary intervention focus), or captured selection or consumption of all manipulated products. If a study included only a category-level measure that captured selection or consumption of a wider set of products beyond those that have been manipulated (but including the manipulated product), this still represented an eligible outcome for the purposes of this review providing it could be meaningfully interpreted at category level, but was considered less desirable because it required assumptions to be made about the direction of effect in relation to the manipulated product itself. Following the application of these criteria, if there remained multiple eligible outcome measures, we selected the single measure of selection or consumption that had been (pre)specified by the study authors as the primary outcome. If no primary outcome had been specified by study authors, we selected the measure of selection or consumption most proximal to health outcomes in the context of the specific intervention. For example, if a study reported measures of both energy intake and the amount of food eaten (in grams), we selected energy intake as the measure most proximal to diet-related health outcomes, and where measures were reported relating to both intake of a healthier (e.g. low energy density) product and intake of a less-healthy (e.g. high energy density) product, we prioritised the latter.

For all outcome data, we collected information on: outcome variable type (dichotomous, continuous); outcome variable definition; unit of measurement (if relevant); timing of measurement (immediate ( $\leq 1$ day) or longer term ( $>1$ day)); and type of measure (objective, self-report). For dichotomous outcomes, we extracted event rates in each comparison group. For continuous outcomes, we extracted mean differences, or mean changes in final measurements from baseline measurements, for each comparison group with associated standard deviations (or if standard deviations were missing, standard errors, 95\% confidence intervals or relevant t-statistics, F-statistics, or $\mathrm{P}$ values). For included studies using factorial designs to investigate the effects of multiple experimental manipulations, we combined groups to capture the main effects of each relevant randomised comparison. 


\section{Assessment of risk of bias in included studies}

We assessed risk of bias in the included studies using the revised Cochrane 'Risk of bias' tool for randomised trials (RoB 2.0) (Higgins 2016a), employing the additional guidance for clusterrandomised and cross-over trials (Eldridge 2016; Higgins 2016b). RoB 2.0 addresses five specific domains: (1) bias arising from the randomisation process; (2) bias due to deviations from intended interventions; (3) bias due to missing outcome data; (4) bias in measurement of the outcome; and (5) bias in selection of the reported result. Two review authors independently applied the tool to each included study, and recorded supporting information and justifications for judgements of risk of bias for each domain (low; high; some concerns). Any discrepancies in judgements of risk of bias or justifications for judgements were resolved by discussion to reach consensus between the two review authors, with a third review author acting as an arbiter if necessary. Following guidance given for RoB 2.0 (Section 1.3.4) (Higgins 2016a), we derived an overall summary 'Risk of bias' judgement (low; some concerns; high) for each specific outcome, whereby the overall RoB for each study was determined by the highest RoB level in any of the domains that were assessed.

\section{Measures of treatment effect}

For continuous outcomes, we calculated the standardised mean difference (SMD) with 95\% confidence intervals (Cls) to express the size of the intervention effect in each study relative to the variability observed in that study. For dichotomous outcomes, we calculated the odds ratio (OR) for each included study to express the size of the relative intervention effect between comparison groups, with the uncertainty in each result being expressed by the $\mathrm{CI}$. We then re-expressed the OR as an SMD by applying the formula described in Section 9.4.6 of the Cochrane Handbook for Systematic Reviews of Interventions (Deeks 2011). We calculated SMDs and sampling variances using means, standard deviations (SDs), and sample sizes and the corresponding equations for continuous and dichotomous data. We extracted means and SDs from published figures if they had not been reported numerically. If SDs were not reported or available from authors, they were obtained using the first appropriate rule from the following:

(a) by direct calculation from statistics such as standard errors or Cls, if available;

(b) by imputation, by assuming that the ratio of SD to mean for the outcome is equal to the ratio of SD to mean calculated using raw data available for other outcomes in the same study;

(c) by imputation, by assuming that the ratio of SD to mean is equal to the ratio of SD to mean observed in other similar studies that reported both means and SDs.

\section{Unit of analysis issues}

In the case of cluster-randomised trials, where an analysis was reported that accounted for the clustered study design, we estimated the effect on this basis, using reported test statistics (t-statistics, F-statistics or P values) to calculate standard errors. When this was not possible and the information was not available from the authors, we carried out an 'approximately correct' analysis according to current guidelines in Section 16.3 of the Cochrane Handbook for Systematic Reviews of Interventions (Higgins 2011). We imputed estimates of the intracluster correlation (ICC) using estimates derived from similar studies or by using general recommendations from empirical research. In cases where it was not possible to implement these procedures, we gave the effect estimate as presented but have reported the unit of analysis error.

For included studies with a within-participants design, we aimed to account for the design by calculating the SMD for continuous outcomes using the methods described in Section 16.4 of the Cochrane Handbook for Systematic Reviews of Interventions (Higgins 2011), where standard errors for outcome data are computed using reported test statistics or estimates of correlations. However, adjustments could not be made to standard errors to account for within-subject designs as suitable information about within-person correlations was not available in the studies. None of the withinsubject studies reported SDs directly, and we were already making strong assumptions to estimate the missing SDs. In order to adjust for the within-subject design, additional unsupported assumptions regarding the correlations would need to have been made.

Final outcome values served as the primary unit of analysis. For studies assessing changes from baseline as a result of an experimental manipulation, we calculated final values based on either reported data or supplementary data obtained by contacting the study authors.

In relation to potential unit of analysis issues arising from studies with multiple eligible comparison groups, our plans are provided below in the Data synthesis section.

\section{Dealing with missing data}

We sought data that were missing from reports of included studies by contacting the study authors. Where data were missing due to participant dropout, we conducted available-case analyses and recorded any issues related to missing data within the 'Risk of bias' assessment.

\section{Assessment of heterogeneity}

We assessed statistical heterogeneity in results by inspecting a graphical display of the estimated treatment effects from included studies along with their $95 \% \mathrm{Cls}$, and by formal statistical tests of homogeneity $\left(\mathrm{Chi}^{2}\right)$ and measures of inconsistency (12) and heterogeneity $\left(\operatorname{Tau}^{2}\right)$.

\section{Assessment of reporting biases}

We drew funnel plots (plots of effect estimates versus the inverse of their standard errors) to inform assessment of reporting biases. We conducted statistical tests to formally investigate the degree of asymmetry using the method proposed by Egger and colleagues (Egger 1997). Results of statistical tests were interpreted based on visual inspection of the funnel plots. Asymmetry of the funnel plot may indicate publication bias or other biases related to sample size, though it may also represent a true relationship between trial size and effect size.

\section{Data synthesis}

We described and summarised the findings of included studies to address the objectives of the review. We provided a narrative synthesis describing the interventions, participants, study characteristics, and effects of eligible interventions upon prespecified outcomes (see Criteria for considering studies for this review). Our statistical analysis of the results of included studies 
used a series of random-effects and fixed-effect models to estimate summary effect sizes as SMDs with $95 \% \mathrm{Cls}$ in terms of each specified outcome. Our planned statistical analysis comprised the following stages:

- Stage 1: conduct separate meta-analyses for each product type (food, alcohol, and tobacco) and, within each product type, conduct separate meta-analyses for (i) availability interventions and (ii) proximity interventions.

Then for each meta-analysis:

- Stage 2: conduct a meta-regression analysis with study characteristics (including summary risk of bias) as covariates;

- Stage 3: conduct a meta-regression analysis with intervention characteristics as covariates;

- Stage 4: conduct a meta-regression analysis with participant characteristics as covariates.

Study-level effect sizes calculated based on outcome data from independent within-study comparisons were directly incorporated into Stage 1 meta-analyses. For studies that included three or more eligible comparison groups (e.g. a study of a proximity intervention placing a food product either $1 \mathrm{~m}, 2 \mathrm{~m}$, or $3 \mathrm{~m}$ from participants), we treated each eligible within-study comparison as providing independent outcome data, but adjusted those data to account for the dependency between multiple comparisons as described in the following paragraph. We planned to analyse data from multi-arm studies based on incremental comparisons only. We followed this for availability interventions, whilst for the analysis of proximity interventions, we decided to consider the shortest distance as a comparator against which all other intervention arms would be compared (e.g. $1 \mathrm{~m}$ versus $2 \mathrm{~m}, 1 \mathrm{~m}$ versus $3 \mathrm{~m}$, but not $2 \mathrm{~m}$ versus 3 $\mathrm{m})$, to allow meta-regression analyses to investigate the impact of increasing differences in proximity and because the nearest point to the participant is the logical comparator in any comparison given the mechanisms posited to underlie the intervention.

For studies contributing multiple pairwise comparisons to a metaanalysis, provided the sample size was large enough, each pairwise comparison was included separately. We adjusted the study weights to account approximately for the statistical dependencies between comparisons by dividing the sample size of the common intervention group as evenly as possible between the comparisons. If the sample size for the common intervention group was 1 for any comparison after dividing the common intervention group, we combined intervention groups to give a single pairwise comparison. If there was an even number of groups, we divided the groups in half based on the level (i.e. low or high) of the intervention type (i.e. proximity and availability). If the number of groups was odd, the group left over as the 'middle' level was incorporated into the higher-level group. We did not undertake multivariate analysis to deal with studies with multiple treatment arms as had been proposed as a possibility in the protocol, since the studies with multiple treatment arms had different numbers of arms that were not directly comparable across studies. As a post hoc sensitivity analysis, we repeated meta-analyses but instead entered a single effect estimate for each multi-arm study, obtained using the mean SMD and the mean variance across the multiple comparisons from that study (a conservative approach that will underestimate precision).
We planned to exclude a covariate from Stages 2, 3, or 4 of a meta-regression analysis if useable data were available from fewer than 10 eligible studies incorporated into the corresponding Stage 1 meta-analysis and/or covariate values did not enable sufficient discrimination between studies (e.g. if covariates are identical, with all included studies using a between-participants design and randomising individual participants). Within each stage of a meta-regression analysis, we proposed to test each covariate separately to identify those variables statistically associated with each outcome. Finally, we planned to estimate and present a metaregression model that incorporated the set of covariates that best explained statistical heterogeneity observed in the corresponding Stage 1 meta-analysis. We planned to use the following procedure to select and incorporate covariates into this multivariable model:

1. rank those covariates identified as potentially important predictors of the outcome in Stages 2, 3, or 4 in order of the corresponding adjusted $\mathrm{R}^{2}$ values;

2. starting with the top-ranked covariate, use a stepwise procedure to add each consecutively ranked covariate into the multivariable meta-regression model; and

3. retain a covariate in the multivariable model only if it increases the adjusted $R^{2}$ for the multivariable and no collinearity or multicollinearity with other retained covariates is detected.

In practice, we conducted meta-regression only on the consumption outcome for proximity interventions, as there were insufficient data (fewer than 10 comparisons) for all other interventions/outcomes. Furthermore, we conducted only univariate meta-regression analyses. Multivariate analyses were not possible due to a lack of data and given that there were not variables identified that modified the intervention effect within each stage of the analysis. Additional details and results of the meta-regression analyses are reported in the Effects of interventions section.

Statistical analyses were conducted in $\mathrm{R}$ (version 3.4.2) and metafor (version 2.0-0) (Viechtbauer 2010).

\section{Treatment of multicomponent studies}

For included studies using factorial designs to investigate the effects of multiple experimental manipulations, we combined outcome data across groups to capture the main effect attributable to each 'availability' or 'proximity' comparison. For studies of interventions with concurrent components that were unrelated to but intrinsically confounded with the manipulations of interest (namely product availability or proximity), we treated the presence of concurrent components as a study characteristic, indicating the presence or absence of one or more additional intervention components. An example of such confounded concurrent components would be when a product is made less available but also has warning labels added to its packaging (relative to that product being more available and having no additional warning labels). Our primary analyses excluded comparisons where confounded concurrent intervention components were present. We subsequently conducted sensitivity analyses whereby these comparisons were reinstated, in order to assess their impact on the results. 


\section{Certainty of evidence}

We used the GRADE framework to rate the certainty of each body of evidence incorporated into meta-analyses for (1) selection (with or without purchasing) and (2) consumption outcomes, to indicate the confidence that can be placed in summary estimates of effect (Guyatt 2011; Schünemann 2011). This is an assessment of the likelihood that the true effect will not be substantially different from what the research found. Within the GRADE approach, the certainty of a body of evidence for intervention effects is assessed based on the design of the underlying studies - with RCTs initially considered high certainty - and on a number of factors that can decrease or increase certainty. GRADE criteria for downgrading certainty of evidence encompass study limitations, inconsistency, imprecision, indirectness, publication bias, and other considerations (Balshem 2011). If such a criterion is identified, it is classified either as serious (leading to downgrading by one level) or very serious (downgrading by two levels). The four possible certainty ratings that can be applied range from high certainty (meaning that current evidence provides a very good indication of the likely effect, and the likelihood that the actual effect will be substantially different is low) through to moderate certainty (current evidence provides a good indication of the likely effect, and the likelihood that the actual effect of the treatment will not be substantially different is moderate); low certainty (current evidence provides some indication of the likely effect, but the likelihood that the actual effect will be substantially different is high); and very low certainty (current evidence does not provide a reliable indication of the likely effect, and the likelihood that the actual effect will be substantially different is very high). Two review authors independently undertook duplicate assessment of GRADE, with any disagreements resolved by discussion or by consulting a third review author if necessary to reach consensus.

\section{'Summary of findings' tables}

We developed 'Summary of findings' tables using GRADEpro GDT (GRADEpro GDT 2015). These tables comprise summaries of the estimated intervention effect and the number of participants and studies for each primary outcome, and include justifications underpinning GRADE assessments. We planned to present separate summary effect sizes and certainty of evidence ratings for food, alcohol, and tobacco products, and for availability and proximity interventions within each of these product types, but in practice no eligible alcohol or tobacco studies were identified. Results of random-effects meta-analyses are presented as SMDs with $95 \% \mathrm{Cls}$. To facilitate interpretation of these estimated effect sizes, we reexpressed them employing selected familiar metrics of selection or consumption using observational data from a populationrepresentative sample (see Effects of interventions for details) (Hollands 2015; Schünemann 2011).

\section{Sensitivity analysis}

In addition to the aforementioned treatment of studies featuring confounded additional intervention components, we also conducted sensitivity analyses to explore the impact of any outcome data that were imputed due to missing data.

\section{RE S U L T S}

\section{Description of studies}

\section{Results of the search}

The flow of studies through the systematic review process is shown in Figure 2. Electronic database searches were initially run between 1 and 4 March 2016. These retrieved a total of 233,996 study records, including duplicates. Twenty-four additional records in the review had been previously identified from other sources, functioning as a reference set, resulting in a total of 234,020 records. Following removal of duplicates (76,899 records), 157,121 titleabstract records were processed in accordance with the semiautomated screening workflow described in Appendix 2 . As a result of this process, 27,116 title and abstract records were screened, of which 121 articles were subject to full-text screening. The electronic database searches were updated between 23 and 27 July 2018. For these updated searches, following removal of 7202 duplicate records, 37,864 title-abstract records were processed in accordance with the semi-automated workflow. This resulted in 2962 titleabstract records being screened, with a further nine articles subject to full-text screening. 
Figure 2. Study flow diagram.

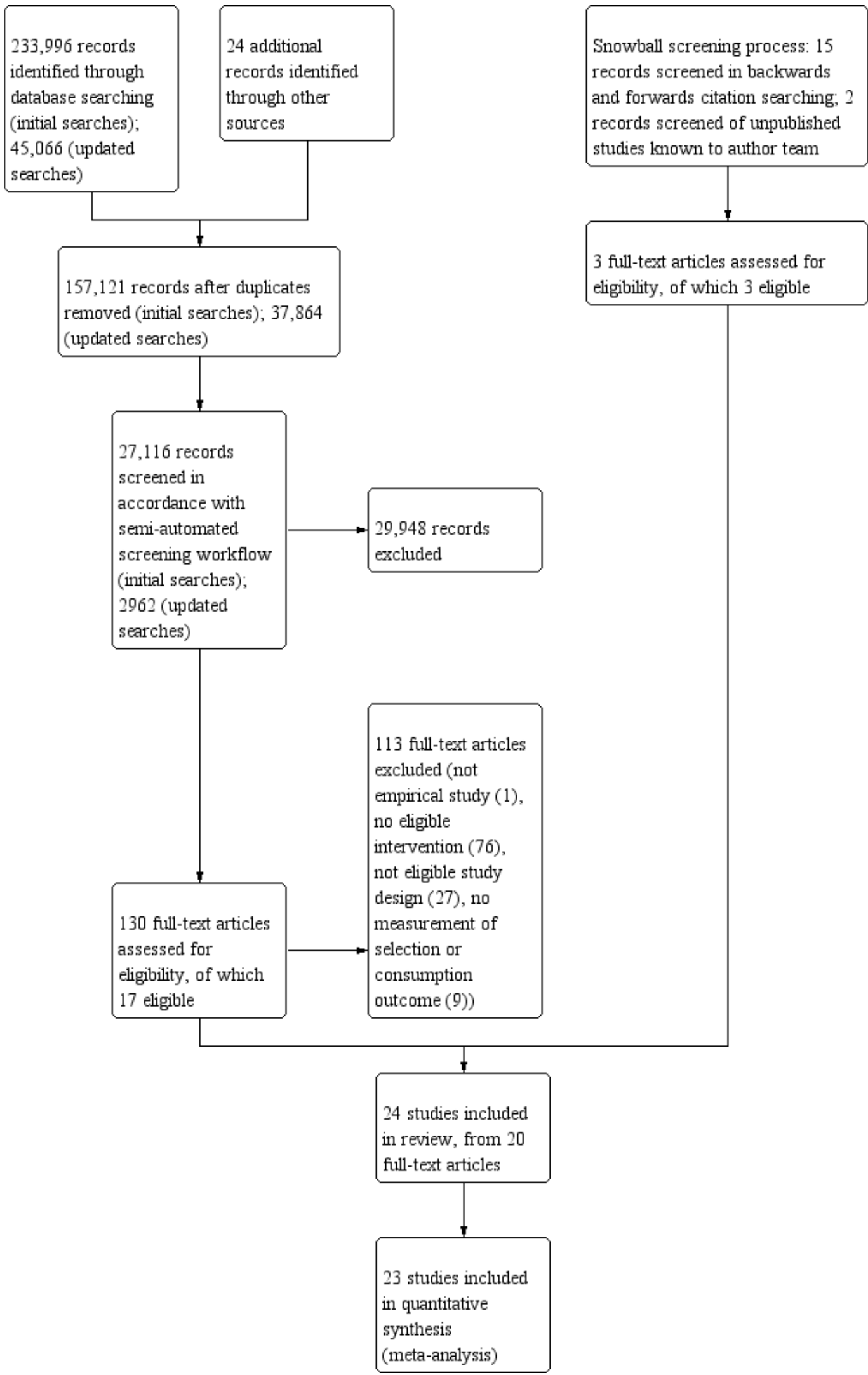


At full-text screening stage, we excluded 113 articles and assessed 17 articles assessed as eligible for inclusion in the review. These 17 articles represent 20 unique studies ( 6 availability: Fiske 2004; Foster 2014; Kocken 2012; Pechey 2019; Roe 2013; Stubbs 2001; and 14 proximity: Cohen 2015; Engell 1996 (S1); Engell 1996 (S2); Greene 2017; Langlet 2017; Maas 2012 (S1); Maas 2012 (S2); MusherEizenman 2010; Painter 2002; Privitera 2012 (S1); Privitera 2012 (S2); Privitera 2014; Wansink 2006; Wansink 2013a). Snowball screening conducted between 31 October and 2 November 2016 and again between 9 and 12 November 2018 resulted in the identification of four further studies from three full-text articles, with one study of proximity identified through backward and forward citation searching (Kongsbak 2016), and three studies of proximity identified due to two review authors being authors on those studies, which have subsequently been published (Hunter 2018 (S1); Hunter 2018 (S2); Hunter 2019). We included a total of 24 studies in the review.

We identified registered protocols for four ongoing studies (see Characteristics of ongoing studies), and there were insufficient details available to determine eligibility for a further two studies (see Characteristics of studies awaiting classification).

\section{Included studies}

We included 24 studies involving a total of 3052 participants in the review. Fourteen studies were conducted in the USA (Cohen 2015; Engell 1996 (S1); Engell 1996 (S2); Fiske 2004; Foster 2014; Greene 2017; Musher-Eizenman 2010; Painter 2002; Privitera 2012 (S1); Privitera 2012 (S2); Privitera 2014; Roe 2013; Wansink 2006; Wansink 2013a); five in the UK (Hunter 2018 (S1); Hunter 2018 (S2); Hunter 2019; Pechey 2019; Stubbs 2001); three in the Netherlands (Kocken 2012; Maas 2012 (S1); Maas 2012 (S2)); one in Denmark (Kongsbak 2016); and one in Sweden (Langlet 2017). We identified no eligible studies conducted in LMICs.

The majority $(14 / 24)$ of the included studies were conducted in laboratory settings (Engell 1996 (S1); Engell 1996 (S2); Hunter 2018 (S1); Hunter 2018 (S2); Hunter 2019; Kongsbak 2016; Langlet 2017; Maas 2012 (S1); Maas 2012 (S2); Musher-Eizenman 2010; Privitera 2012 (S1); Privitera 2012 (S2); Privitera 2014; Stubbs 2001); the remaining 10 studies (five availability, Fiske 2004; Foster 2014; Kocken 2012; Pechey 2019; Roe 2013, and five proximity, Cohen 2015; Greene 2017; Painter 2002; Wansink 2006; Wansink 2013a) were conducted in a wide range of field settings including shops, restaurants/cafeterias, offices, and vending machines in schools. Study participants in 17 studies were - or were assumed to be adults (Engell 1996 (S1); Engell 1996 (S2); Fiske 2004; Hunter 2018 (S1); Hunter 2018 (S2); Hunter 2019; Kongsbak 2016; Maas 2012 (S1); Maas 2012 (S2); Painter 2002; Pechey 2019; Privitera 2012 (S1); Privitera 2012 (S2); Privitera 2014; Stubbs 2001; Wansink 2006; Wansink 2013a), and in six studies children under 18 years (Cohen 2015; Greene 2017; Kocken 2012; Langlet 2017; Musher-Eizenman 2010; Roe 2013). Ages were not sufficiently specified in one study to allow classification (Foster 2014). The sex of participants was reported in 15 studies (ranging from 0\% female (Engell 1996 (S1); Engell 1996 (S2); Kongsbak 2016; Stubbs 2001), to 100\% female (Maas 2012 (S1); Maas 2012 (S2); Wansink 2006)), with this unspecified in nine studies (Fiske 2004; Foster 2014; Greene 2017; Kocken 2012; Musher-Eizenman 2010; Pechey 2019; Privitera 2012 (S1); Privitera 2012 (S2); Wansink 2013a). Eleven studies reported BMI. Mean BMI of the sample was < 25 in six studies (Hunter 2018 (S1); Kongsbak 2016; Langlet 2017; Maas 2012 (S1); Maas 2012 (S2);
Musher-Eizenman 2010), and between 25 and 30 in the remaining five studies (Hunter 2018 (S2); Hunter 2019; Privitera 2012 (S1); Privitera 2012 (S2); Stubbs 2001).

In terms of socioeconomic status context of the study samples, five studies were conducted in samples purposefully comprising both high and low deprivation (Greene 2017; Hunter 2018 (S1); Hunter 2018 (S2); Hunter 2019; Pechey 2019); three were conducted in high-deprivation contexts (Cohen 2015; Foster 2014; Kocken 2012); and the remaining 16 studies were conducted in low deprivation contexts (Engell 1996 (S1); Engell 1996 (S2); Fiske 2004; Kongsbak 2016; Langlet 2017; Maas 2012 (S1); Maas 2012 (S2); MusherEizenman 2010; Painter 2002; Privitera 2012 (S1); Privitera 2012 (S2); Privitera 2014; Roe 2013; Stubbs 2001; Wansink 2006; Wansink 2013a).

All 24 included studies involved manipulations of food products, with no eligible studies focused on alcohol or tobacco products.

Six of the included studies concerned manipulations of availability (Fiske 2004; Foster 2014; Kocken 2012; Pechey 2019; Roe 2013; Stubbs 2001). In terms of the types of availability interventions used, two studies changed the absolute number of different options available (Roe 2013; Stubbs 2001), and four studies changed the relative number (proportion) of less-healthy (to healthier) options (Fiske 2004; Foster 2014; Kocken 2012; Pechey 2019). Of the two studies that changed absolute numbers of options, Roe 2013 decreased the number of different fruit and vegetables options offered at a snack occasion, and Stubbs 2001 decreased the total number of different meal options available to participants. Of the four studies that changed relative proportions, two studies decreased (Fiske 2004; Kocken 2012), respectively, the number of high-fat and high-calorie options available in vending machines (with corresponding increases in low-fat and low-calorie options). One study decreased the number of highercalorie (relative to lower-calorie) beverages on display within supermarket checkout refrigerators (Foster 2014). The remaining study decreased the proportion of less-healthy (i.e. higher-energy) cooked meal, snack, cold drink, and sandwich options, with a corresponding increase in healthier (i.e. lower energy) options (Pechey 2019). Two availability studies were confounded to some degree with other concurrent interventions within the same physical environment (Fiske 2004; Foster 2014), the former also manipulating the addition of labels, and the latter additionally manipulating the visibility of products (but not meeting our criteria for a proximity intervention). It should be noted that, in order to ensure consistency in treatment and interpretation of effects, the comparisons within our analysis were always coded as reducing the availability of products, irrespective of whether the intervention was conceptualised by the authors as concerning increasing or decreasing availability. Most studies (4/6) manipulated snack foods or drinks (Fiske 2004; Foster 2014; Kocken 2012; Roe 2013). The included studies of availability investigated intervention exposures over extended time periods varying between 27 days (Stubbs 2001), and six months (Foster 2014). Three availability studies used randomised between-participants designs (Fiske 2004; Foster 2014; Kocken 2012), and three used randomised within-participants designs (Pechey 2019; Roe 2013; Stubbs 2001).

Eighteen of the included studies concerned manipulations of proximity (Cohen 2015; Engell 1996 (S1); Engell 1996 (S2); Greene 2017; Hunter 2018 (S1); Hunter 2018 (S2); Hunter 2019; Kongsbak 2016; Langlet 2017; Maas 2012 (S1); Maas 2012 (S2); Musher- 
Eizenman 2010; Painter 2002; Privitera 2012 (S1); Privitera 2012 (S2); Privitera 2014; Wansink 2006; Wansink 2013a). In terms of the types of interventions used, most studies (14/18) increased the distance at which the product was placed from a set point, in all cases being the distance from a chair, table, or desk where a participant was positioned (Engell 1996 (S1); Engell 1996 (S2); Hunter 2018 (S1); Hunter 2018 (S2); Hunter 2019; Langlet 2017; Maas 2012 (S1); Maas 2012 (S2); Musher-Eizenman 2010; Painter 2002; Privitera 2012 (S1); Privitera 2012 (S2); Privitera 2014; Wansink 2006). All of these studies manipulated snack foods or drinks intended for immediate consumption. In these studies, the comparisons concerned relatively small distances, with the greatest distance products were placed at ranging from $0.7 \mathrm{~m}$, Hunter 2018 (S1); Hunter 2018 (S2); Hunter 2019, to 12.2 m, Engell 1996 (S1). Four further studies manipulated the order of products encountered along buffet or lunch lines to increase the distance of the product upon entering that line (Cohen 2015; Greene 2017; Kongsbak 2016; Wansink 2013a), all of which manipulated components of breakfast or lunch meals. Three proximity studies were confounded to some degree with other concurrent interventions within the same physical environment (Cohen 2015; Greene 2017; Kongsbak 2016). Cohen 2015 additionally manipulated placing fruit in attractive containers and other fruit options next to the cash registers, as well as prominently displaying signage promoting fruits and vegetables. Greene 2017 made various changes including to the way in which fruits were presented and labelled. Kongsbak 2016 also manipulated whether salad components were mixed together or placed separately. Similar to our availability analysis, comparisons were always coded as reducing the proximity of products, irrespective of whether the intervention was conceptualised by the authors as concerning increasing or decreasing proximity. The included studies of proximity focused on a range of products that can be characterised as healthier in six studies (fruit, vegetables, water) (Cohen 2015; Engell 1996 (S1); Engell 1996 (S2); Greene 2017; Privitera 2012 (S1); Privitera 2012 (S2)); less healthy in six studies (chocolate) (Hunter 2018 (S1); Hunter 2018 (S2); Maas 2012 (S1); Maas 2012 (S2); Painter 2002; Wansink 2006); and a mix of healthier and less healthy in five studies (Hunter 2019; Kongsbak 2016; Langlet 2017; Musher-Eizenman 2010; Privitera 2014; Wansink 2013a). Studies typically investigated intervention exposures that were one-off or, if repeated, were repeated over relatively short time periods. Only four studies involved exposing participants to interventions for a period of more than one day (Cohen 2015; Greene 2017; Painter 2002; Wansink 2006). Sixteen proximity studies used randomised between-participants designs (Cohen 2015; Engell 1996 (S1); Engell 1996 (S2); Greene 2017; Hunter 2018 (S1); Hunter 2018 (S2); Hunter 2019; Kongsbak 2016; Langlet 2017; Maas 2012 (S1); Maas 2012 (S2); Musher-Eizenman 2010; Privitera 2012 (S1); Privitera 2012 (S2); Privitera 2014; Wansink 2013a), and two used randomised within-participants designs (Painter 2002; Wansink 2006).

The majority of studies (15/24) reported sources of funding. There was no apparent conflict of interest in the funding of 12 studies, given explicit statements denying involvement by agencies with possible commercial conflicts of interest in their results (Cohen 2015; Foster 2014; Greene 2017; Hunter 2018 (S1); Hunter 2018 (S2); Hunter 2019; Langlet 2017; Pechey 2019; Privitera 2012 (S1); Privitera 2012 (S2); Roe 2013; Wansink 2013a). This was unclear in the remaining 12 studies.
Further details on characteristics of interventions and comparators are provided in the Characteristics of included studies section.

We contacted the authors of eight studies with requests for data or clarifications on presented data (Fiske 2004; Langlet 2017; MusherEizenman 2010; Painter 2002; Pechey 2019; Stubbs 2001; Wansink 2006; Wansink 2013a). We received data from Langlet 2017 and Pechey 2019, while we included Musher-Eizenman 2010 in analysis using data provided in the report. Regarding the remaining five studies, we imputed standard deviations for four studies using methods described in the Measures of treatment effect section (Fiske 2004; Painter 2002; Stubbs 2001; Wansink 2006). We excluded Wansink 2013a from the analysis due to reporting data that were modelled, with the raw data based on observations unobtainable.

\section{Excluded studies}

We excluded 113 studies at the full-text screening stage. Of these, we excluded 76 studies for not featuring an eligible intervention; 27 for not using an eligible study design; nine for not assessing selection or consumption as defined; and one study for not being a report of an empirical study. Excluded studies are listed in the Characteristics of excluded studies section.

\section{Risk of bias in included studies}

We used the RoB 2.0 tool to assess risk of bias for each of the included studies. A summary of these assessments is provided in Table 1. In terms of overall risk of bias, there were concerns about risk of bias for the majority of studies (20/24), with two of these assessed as at high risk of bias (Musher-Eizenman 2010; Wansink 2013a). A text summary is provided below for each of the six individual components of the 'Risk of bias' assessment.

Bias arising from the randomisation process. This was the most common criterion for which risk of bias was apparent, with significant concerns regarding 19/24 studies (Cohen 2015; Engell 1996 (S1); Engell 1996 (S2); Fiske 2004; Greene 2017; Kocken 2012; Kongsbak 2016; Langlet 2017; Maas 2012 (S1); Maas 2012 (S2); Musher-Eizenman 2010; Painter 2002; Pechey 2019; Privitera 2012 (S1); Privitera 2012 (S2); Privitera 2014; Roe 2013; Stubbs 2001; Wansink 2006), due primarily to an absence of detail in describing the randomisation and allocation concealment processes. We considered one study as at high risk of bias because it could not be ruled out based on the description that participants were allocated to intervention in an alternating sequence rather than as a result of randomisation (Wansink 2013a).

Bias arising from the timing of identification and recruitment of individual participants in relation to timing of randomisation. This assessment only applied to cluster-randomised trials (Cohen 2015; Fiske 2004; Foster 2014; Kocken 2012; Langlet 2017), and all five such studies were considered as at low risk of bias for this domain.

Bias due to deviations from intended interventions. We judged all studies to be at low risk of bias on this domain, apart from two studies for which significant concerns were identified (Painter 2002; Wansink 2006). These two studies used cross-over designs with short wash-out periods, meaning that it is likely that participants were aware of being exposed to the intervention and the possibility of carry-over effects could not therefore reasonably be dismissed.

Bias due to missing outcome data. This was typically judged to be at low risk. There were substantive concerns about risk of bias for 
one study in which there were substantial missing data (MusherEizenman 2010). Additionally, the assignment and effect of missing participants was not detailed.

Bias in measurement of the outcome. All of the included studies used objective measures of behaviour, an inclusion criterion for the review. We judged this domain as low risk for all studies.

Bias in selection of the reported result. We judged this domain as low risk for all studies. However, it is notable that, to our knowledge, only five of the included studies were preregistered with publicly available study protocols (Cohen 2015; Hunter 2018 (S1); Hunter 2018 (S2); Hunter 2019; Pechey 2019).

\section{Effects of interventions}

See: Summary of findings for the main comparison Lower versus higher availability (i.e. fewer versus more options) of food products for changing quantity of food selected or consumed; Summary of findings $\mathbf{2}$ Lower versus higher proximity (i.e. placed farther away versus placed nearer) of food products for changing quantity of food selected or consumed

We have reported results separately for both availability interventions and proximity interventions, for both selection and consumption outcomes. As we identified no study in which alcohol or tobacco products were the target of the intervention, the results of this review relate solely to food. Results of meta-analyses are presented as standardised mean differences (SMDs) with 95\% confidence intervals (Cls). Using a similar approach to re-expressing effect sizes in a more familiar metric as we have used previously (Crockett 2018; Hollands 2015), we have also re-expressed SMDs based on estimated average (mean) consumption levels and standard deviations (SDs) among representative samples of the UK population. For this translation, we calculated an estimate of the percentage reduction in energy consumed over a typical snack occasion, given that the included studies typically manipulated snack foods intended for immediate consumption. For selection outcomes, it was assumed that all food that is selected is consumed. We used a mean (SD) of $200( \pm 63)$ kilocalories(kcal) as a baseline value, given guidance concerning a $200 \mathrm{kcal}$ threshold for energy consumption from snacks (NHS 2018). These figures were based on daily energy intake from food among UK adults (aged 19 to 64 ) estimated at $1773 \pm 561 \mathrm{kcal}$ by the most recent available years (7-8, i.e. $2014 / 15$ to $2015 / 16$ ) of the UK National Diet and Nutrition Survey (Public Health England 2018a). However, it is important to note the limitations of such translations (Hollands 2015). Firstly, these re-expressed values relate to UK populations (although readers could translate the results in similar fashion using representative survey data from other countries). Secondly, because such translations necessarily extrapolate beyond the scope of the included studies and data therein, they are intended only to be illustrative for guiding interpretation of the metaanalyses. In this particular case, there is additional extrapolation in assuming that the variance associated with total daily energy intake will be proportionate for lower levels of energy intake.

\section{Effect on selection of lower availability of food products}

For our primary analysis, useable outcome data were available for three comparisons involving 154 participants and identified from three food studies that changed either the relative number (proportion) of less-healthy (to healthier) options, or changed the absolute number of different options available (Kocken 2012; Pechey 2019; Roe 2013). Kocken 2012 decreased the number of high-calorie options available in vending machines (with corresponding increases in low-calorie options). Pechey 2019 changed the relative proportion of less-healthy (i.e. higher energy) cooked meal, snack, cold drink, and sandwich options, with a corresponding change in healthier (i.e. lower energy) options. Roe 2013 changed the absolute number of different fruit and vegetable options.

Random-effects meta-analysis produced a summary mean effect size (SMD) of $-1.13(95 \% \mathrm{Cl}-1.90$ to $-0.37, \mathrm{P}=0.003)$, meaning that lower availability of a targeted range or category of food(s) here being a lower proportion of less-healthy (to healthier) options or a lower absolute number of different options - decreased the amount that was selected, with a large relative effect size (Figure 3; Summary of findings for the main comparison). Our interpretation of the size of this summary effect suggests that if availability was reduced for an assumed average snack occasion of $200( \pm 63) \mathrm{kcal}$, adults would select $71 \mathrm{kcal}$ fewer, reducing energy selected by $35.6 \%\left(59.9 \%\right.$ to $11.7 \%$ less). The $\mathrm{I}^{2}$ statistic $(64 \%)$ indicates that a substantial amount of the total variance in study-level estimates of this effect was attributable to statistical heterogeneity, which was consistent with differences in the characteristics of the studies within this analysis. When a fixed-effect model was used, the effect was similar: SMD $-1.01(95 \% \mathrm{Cl}-1.35$ to $-0.67, \mathrm{P}<0.001)$. 
Figure 3. Forest plot of the standardised mean difference (SMD) in selection with higher (intervention 1) versus lower (intervention 2 ) availability of food products (i.e. more versus fewer options).

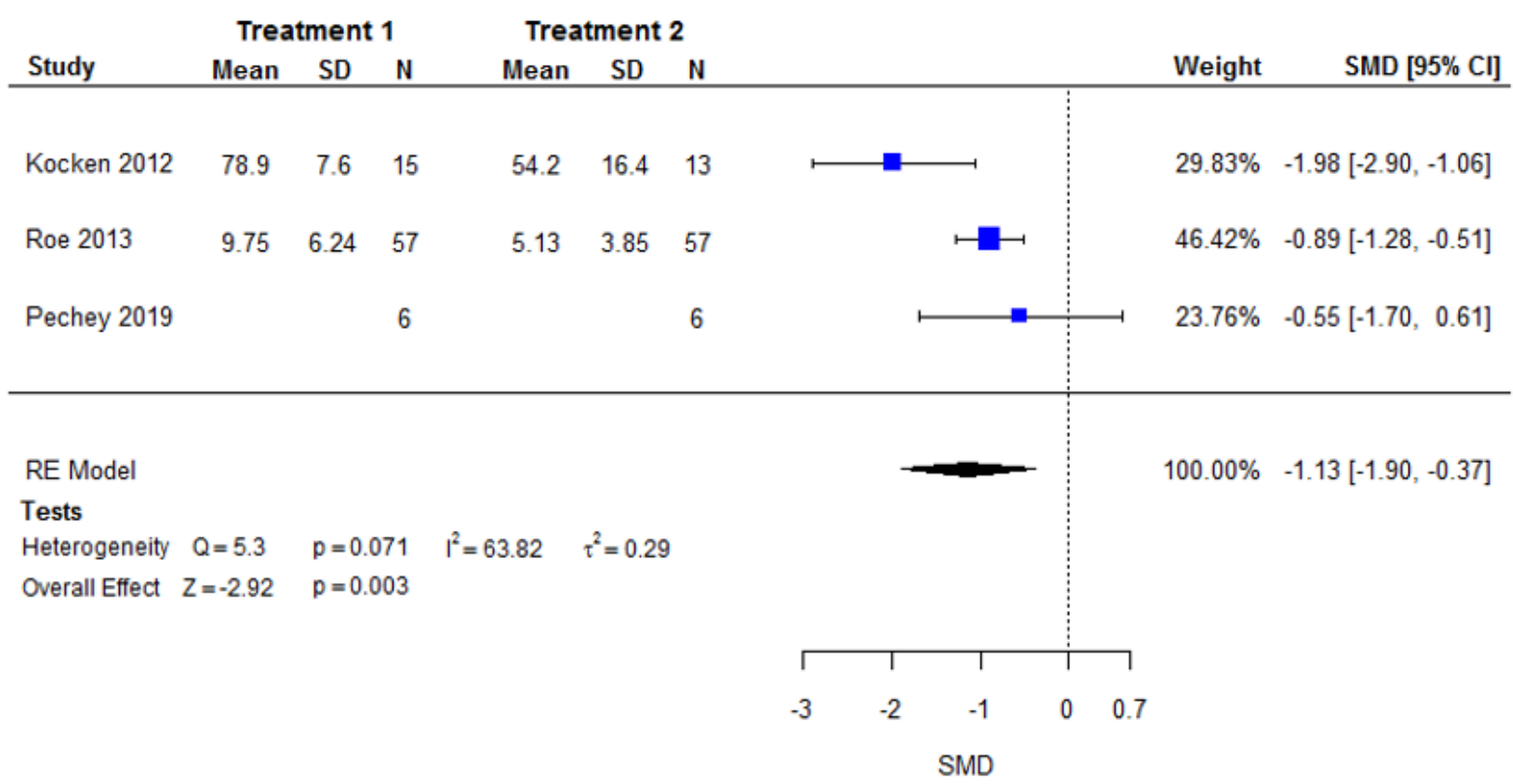

Assessing evidence of possible publication bias via funnel plots was not appropriate due to the low number of studies. We were unable to conduct meta-regression analysis due to the lack of studies, with fewer than 10 data points for all variables.

GRADE assessment indicated that the evidence for this outcome is of low certainty, meaning that current evidence provides some indication of the likely effect, but the likelihood that the actual effect will be substantially different is high. We reached this judgement through consideration of the following criteria. We downgraded the current evidence by one level (i.e. serious limitations) due to study limitations because we judged the studylevel estimate of this effect to have significant concerns related to risk of bias. We also downgraded the evidence by one level due to imprecision, because even though the lower $\mathrm{Cl}$ value indicated a small-moderate effect, the Cls were wide, and the number of participants (sample size) incorporated into this meta-analysis was notably small and did not exceed the optimal information size (i.e. the number of participants generated by a conventional sample size calculation for a single adequately powered trial powered conservatively to detect a small effect size). We did not downgrade the evidence for inconsistency (effect sizes were in a consistent direction with some overlap of $\mathrm{Cls}$, although heterogeneity was considerable), indirectness, or for other considerations including publication bias.

No data were imputed for studies included in the primary analysis, therefore the planned sensitivity analysis concerning imputed data was not applicable. We excluded data from two additional studies from the primary analysis for this outcome due to the intervention effect being confounded as part of multicomponent interventions (Fiske 2004; Foster 2014). In a prespecified sensitivity analysis, we reinstated these data, resulting in an analysis of five comparisons, involving 172 participants, identified from five food studies (Fiske 2004; Foster 2014; Kocken 2012; Pechey 2019; Roe 2013). This did not alter the result or interpretation, with point estimates and $95 \%$ $\mathrm{Cls}$ being similar for both random-effects and fixed-effect models (random-effects SMD: $-1.01(95 \% \mathrm{Cl}-1.57$ to $-0.45, \mathrm{P}<0.001)$; fixedeffect SMD: $-0.97(95 \% \mathrm{Cl}-1.30$ to $-0.65, \mathrm{P}<0.001))$.

\section{Effect on consumption of lower availability of food products}

For our primary analysis, useable outcome data were available for three comparisons involving 150 participants in two food studies that changed the absolute number of different options available (Roe 2013; Stubbs 2001). Roe 2013 changed the absolute number of different fruit and vegetable options, whilst Stubbs 2001 altered the absolute number of different meal options. Random-effects meta-analysis produced a summary mean effect size (SMD) of -0.55 $(95 \% \mathrm{Cl}-1.27$ to $0.18, \mathrm{P}=0.14)$ (Figure 4 ; Summary of findings for the main comparison). This result indicated uncertainty about the effect of lower availability - here being a lower absolute number of different options of a targeted range or category of food(s) - on amount consumed, with the point estimate indicating a moderate reduction in consumption, and wide $\mathrm{Cls}$ that included the possibility of a small increase in consumption. When a fixedeffect model was used, the mean effect size was larger, with $\mathrm{Cls}$ not including zero: $-0.84(95 \% \mathrm{Cl}-1.18$ to $-0.49, \mathrm{P}<0.001)$. Our interpretation of the size of the summary effect from the randomeffects model suggests that if availability was reduced for an assumed average snack occasion of $200( \pm 63) \mathrm{kcal}$, adults would consume $35 \mathrm{kcal}$ fewer, reducing energy consumed by $17.3 \%$ ( $40 \%$ less to $5.7 \%$ more). The $\mathrm{I}^{2}$ statistic (63\%) indicates that a substantial amount of the total variance in study-level estimates of this effect was attributable to statistical heterogeneity, which was consistent with differences in the characteristics of the two studies within this 
analysis. A sensitivity analysis in which a single average SMD was computed for each multi-arm study produced SMD $-0.69(95 \% \mathrm{Cl}$

-1.63 to $0.24, P=0.15)$ in a random-effects analysis based on two results.

\section{Figure 4. Forest plot of the standardised mean difference (SMD) in consumption with higher (intervention 1) versus lower (intervention 2 ) availability of food products (i.e. more versus fewer options).}

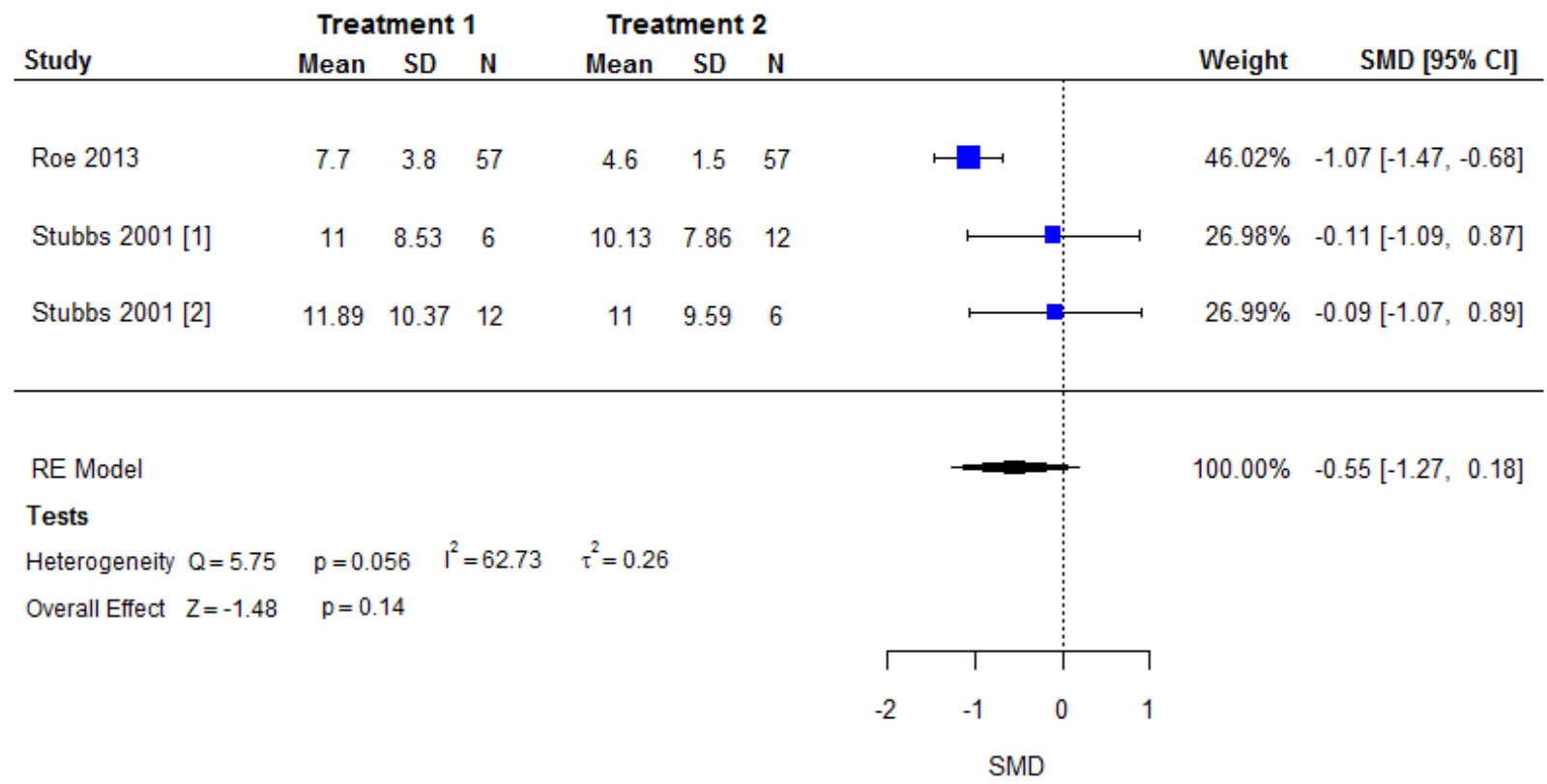

Assessing evidence of possible publication bias via funnel plots was not appropriate due to the low number of studies. We did not conduct meta-regression analyses due to the lack of studies, with fewer than 10 data points for all variables.

GRADE assessment indicated that the evidence for this outcome is of low certainty, meaning that current evidence provides some indication of the likely effect, but the likelihood that the actual effect will be substantially different is high. We reached this judgement through consideration of the following criteria. We downgraded the current evidence by one level (i.e. serious limitations) due to study limitations, as we judged all studylevel estimates of this effect to have significant concerns related to risk of bias. We also downgraded the evidence by one level due to imprecision. Whilst the point estimate indicated a moderate effect on reducing consumption, the $\mathrm{Cls}$ were wide and included the possibility of a small effect on increasing consumption. Furthermore, the number of participants (sample size) incorporated into this meta-analysis was notably small and did not exceed the number of participants generated by a conventional sample size calculation for a single adequately powered trial, powered conservatively to detect a small effect size (optimal information size). We did not downgrade the evidence for inconsistency, as whilst statistical heterogeneity was substantial (although not considerable), effect sizes were in a consistent direction, and the meta-analysis result was driven mainly by a single study (Roe 2013). We also did not downgrade the evidence for indirectness, as all included studies assessed participants, interventions, comparators, and outcomes that met the eligibility criteria for this review. We did not specify that any particular characteristics were more informative than others in addressing the objectives of the review, such as those conducted in particular settings, although the included studies for this outcome were from both field, Roe 2013, and laboratory, Stubbs 2001, settings. Finally, we did not downgrade the evidence for other considerations including publication bias because there was no clear evidence of such bias, and we judged that there were no applicable reasons to consider upgrading the certainty of the evidence.

Our prespecified sensitivity analyses concerning imputed data meant the removal of data from Stubbs 2001, with only the Roe 2013 data remaining, resulting in an effect size (SMD) of -1.07 (95\% Cl -1.47 to -0.68$)$. This analysis did not significantly alter interpretation, and represents only a single study, but relative to our primary analysis, the point estimate indicated a larger reduction in consumption, with wide $\mathrm{Cls}$ that no longer include the possibility of an increase in consumption. The planned sensitivity analysis concerning additional confounded intervention components was not applicable for this outcome. 
Effect on selection of lower proximity (i.e. food products placed farther away)

For our intended primary analysis of selection outcomes for proximity interventions, we identified only one study (Langlet 2017). One comparison (41 participants) found that exposure to food placed farther away resulted in a moderate reduction in its selection: $\mathrm{SMD}-0.65(95 \% \mathrm{Cl}-1.29$ to $-0.01, \mathrm{P}=0.045)$, equivalent to adults selecting $20.5 \%$ less energy ( $40.6 \%$ to $0.3 \%$ less) (Summary of findings 2).

Assessing evidence of possible publication bias via funnel plots was not appropriate due to insufficient studies. Meta-regression analysis could also not be conducted due to insufficient studies, with fewer than 10 data points for all variables.

GRADE assessment indicated that the evidence for this outcome is of very low certainty, meaning that the current evidence does not provide a reliable indication of the likely effect, and that the likelihood that the actual effect will be substantially different is very high. We reached this judgement through consideration of the following criteria. We downgraded the current evidence by one level (i.e. serious limitations) due to study limitations because we judged the study-level estimate of the effect to have significant concerns related to risk of bias. We also downgraded the evidence by one level for imprecision because the effect estimate derived from a single small study. We downgraded the evidence a further level for indirectness, because all the data derived from a study conducted in a laboratory setting, meaning that it may be less directly informative to real-world implementation of the intervention. We did not downgraded the evidence for inconsistency or publication bias.

The planned sensitivity analysis concerning imputed data was not applicable for this outcome. There were additional useable outcome data from three food studies that were excluded from the primary analysis for this outcome due to the intervention effect being confounded as part of multicomponent interventions (Cohen 2015; Greene 2017; Kongsbak 2016). In a prespecified sensitivity analysis, we reinstated these data, resulting in an analysis of four comparisons (1703 participants). This did not result in an interpretation that differed from the primary analysis. Randomeffects meta-analysis produced a summary mean effect size (SMD) of $-0.71(95 \% \mathrm{Cl}-1.08$ to $-0.33, \mathrm{P}<0.001)$, meaning that food products placed farther away resulted in a moderate decrease in selection equivalent to $22.4 \%$ less energy (34\% to $10.4 \%$ less) being selected by adults on each $200 \mathrm{kcal}$ snack occasion. This result was identical when a fixed-effect model was used. The $\mathrm{I}^{2}$ statistic (0\%) indicates that none of the total variance in study-level estimates of this effect was attributable to statistical heterogeneity.

\section{Effect on consumption of lower proximity (i.e. food products placed farther away)}

For our planned primary analysis, outcome data were available for 17 comparisons, involving 1194 participants, identified from 14 food studies (Engell 1996 (S1); Engell 1996 (S2); Hunter 2018 (S1); Hunter 2018 (S2); Hunter 2019; Langlet 2017; Maas 2012 (S1); Maas 2012 (S2); Musher-Eizenman 2010; Painter 2002; Privitera 2012 (S1); Privitera 2012 (S2); Privitera 2014; Wansink 2006). All of these studies increased the distance at which the product was placed from a set point, in all cases being the distance between a chair, table, or desk and where a participant was positioned. Random-effects meta-analysis produced a summary mean effect size (SMD) of $-1.30(95 \% \mathrm{Cl}-2.13$ to $-0.46, \mathrm{P}=0.002)$, meaning that placing food products farther away decreased the amount that was consumed, with a large relative effect size. This result differed in magnitude when a fixed-effect model was used, showing a reduced summary mean effect size (SMD) of -0.55 , with narrower $\mathrm{Cls}(95 \% \mathrm{Cl}-0.68$ to $-0.42, \mathrm{P}<0.001)$. However, the $\mathrm{I}^{2}$ statistic $(97 \%)$ indicates that most of the total variance in study-level estimates of this effect was attributable to statistical heterogeneity, suggesting that the source of this heterogeneity should be identified. Two studies were responsible for a significant proportion of the observed heterogeneity as a result of their outlying respective summary effect sizes of (SMD) -5.34 and -6.96 (Privitera 2012 (S1); Privitera 2012 (S2)). We have found no clear explanation for why these studies would have generated such extreme, heterogeneous estimates: study, intervention, and participant characteristics did not differ notably from other studies included in the analysis. The estimated effect sizes are larger than we consider to be plausible, therefore these data were removed from the main analysis. Removing these two studies meant that this principal analysis involved 15 comparisons (1098 participants) identified from 12 food studies (Engell 1996 (S1); Engell 1996 (S2); Hunter 2018 (S1); Hunter 2018 (S2); Hunter 2019; Langlet 2017; Maas 2012 (S1); Maas 2012 (S2); Musher-Eizenman 2010; Painter 2002; Privitera 2014; Wansink 2006). This resulted in a reduced but still moderate effect size for a random-effects model, with a reduced $\mathrm{I}^{2}$ statistic value of $61 \%$ : SMD $-0.60(95 \% \mathrm{Cl}-0.84$ to -0.36 , $\mathrm{P}<0.001$ ) (Figure 5; Summary of findings 2). Our interpretation of the size of this summary effect suggests that that if proximity was reduced for an assumed average snack occasion of $200( \pm 63)$ $\mathrm{kcal}$, adults would select $38 \mathrm{kcal}$ less, reducing energy consumed by $18.9 \%$ less energy ( $26.5 \%$ to $11.3 \%$ less). We consider this revised analysis of 15 comparisons with outlier values excluded to be the primary analysis for this outcome, and is the result reported in the corresponding Summary of findings 2 and used as the basis for subsequent meta-regression analyses and sensitivity analyses described below. A similar effect was estimated when a fixed-effect model was used: SMD $-0.45(95 \% \mathrm{Cl}-0.58$ to -0.32 , $\mathrm{P}<0.001)$. A sensitivity analysis in which a single average SMD was computed for each multi-arm study produced SMD $-0.59(95 \% \mathrm{Cl}-0.85$ to -0.33 , $\mathrm{P}<0.001)$ in a random-effects analysis based on 12 results. 
Figure 5. Forest plot of the standardised mean difference (SMD) in consumption with higher (intervention 1) versus lower (intervention 2) proximity of food products (i.e. placed nearer versus farther away).

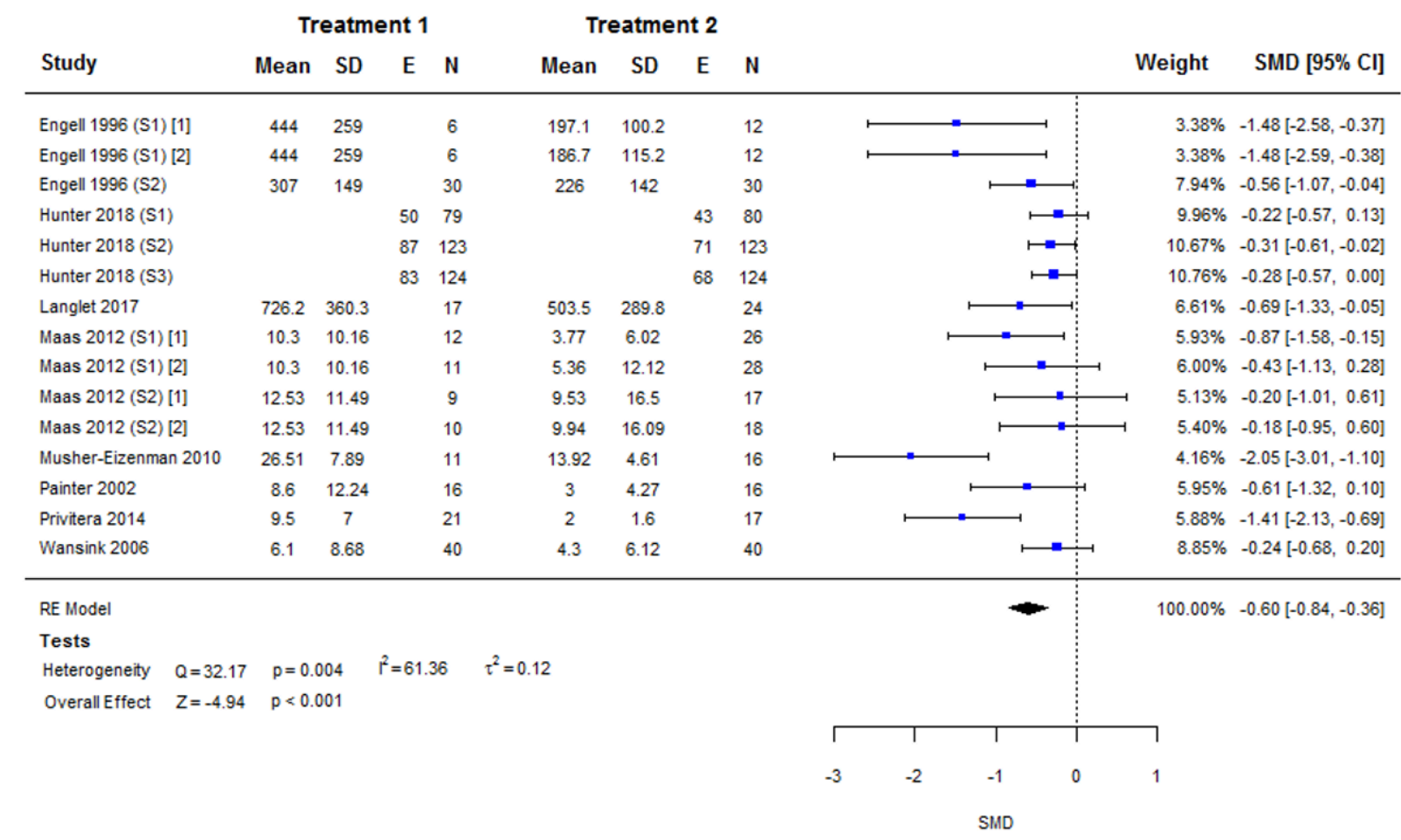

An asymmetrical funnel plot was observed for this analysis (Figure $6)$, with Egger's test giving a $P<0.001(Z=-4.0853)$, suggesting the possible presence of publication bias (since the larger studies have
SMD estimates nearer 0 ). The determination of possible publication bias informed the GRADE assessment for this outcome. 
Figure 6. Funnel plot for meta-analysis of consumption with higher versus lower proximity.

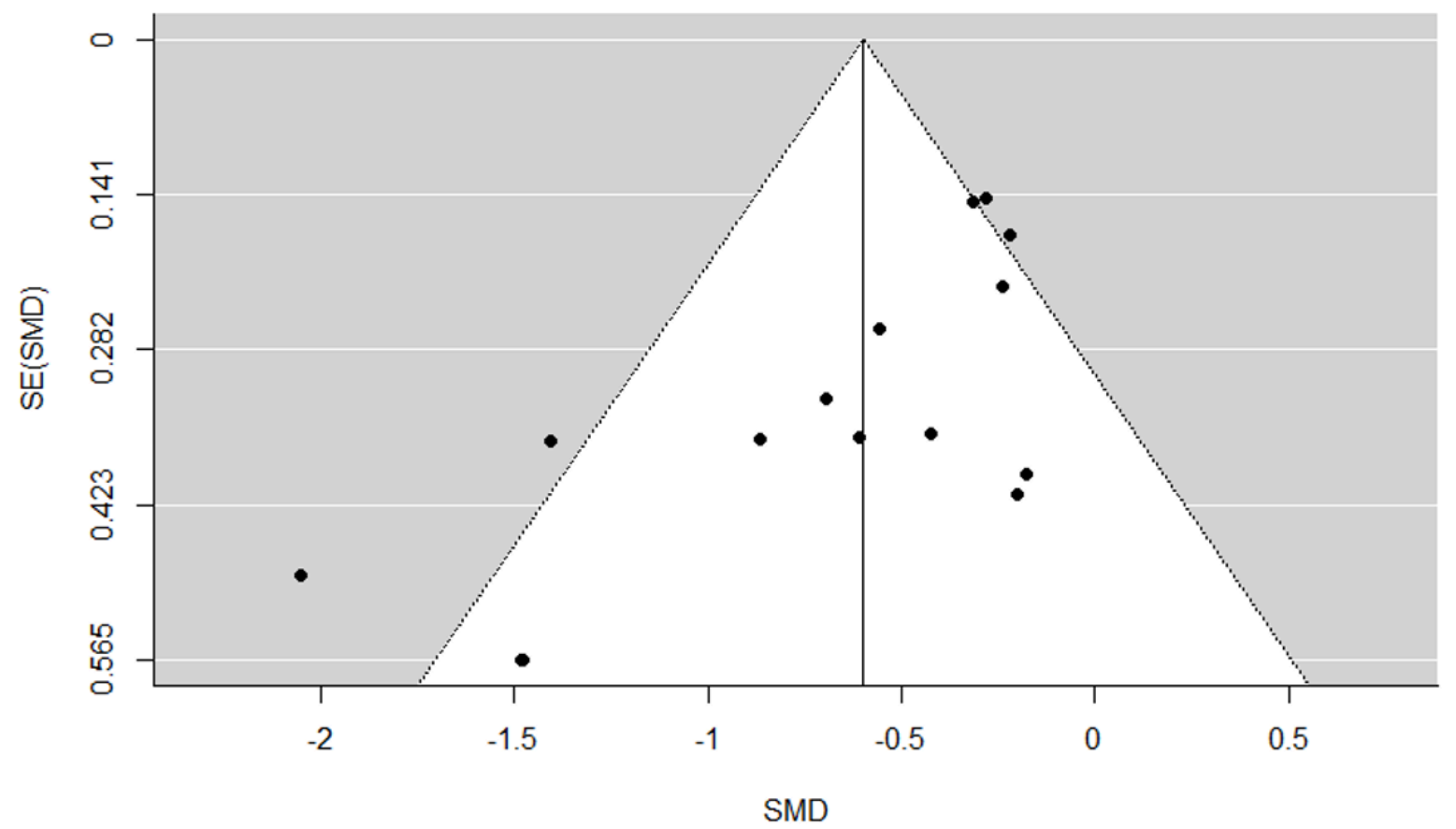

\section{Potential modifiers of the effect on consumption (meta- regression analyses)}

For the effect of proximity on consumption, there were sufficient data as per our criteria (i.e. data points from at least 10 studies for at least some extracted variables) to conduct meta-regression analyses to investigate potential effect modifiers. Whilst the majority of candidate variables were excluded due to either insufficient data or the absence of variability in data values between studies, univariable meta-regression analysis was possible for 10 extracted variables, with numerical results presented in full in Appendix 3. We observed that four of these covariates were associated with the effect of proximity on consumption. We have outlined the stages of the meta-regression analyses below (as described in Data synthesis), and for each stage, have highlighted any variables observed to be associated with the intervention effect.

At Stage 1, we conducted the meta-analysis described above, followed at each subsequent stage with a meta-regression analysis.

At Stage 2, where we examined study characteristics as covariates, the following three variables were significantly associated with the intervention effect.

- Product-outcome relationship. Effect sizes for lower (versus higher) proximity were larger when the specific product(s) that was manipulated was the only product available to participants, as opposed to there being other unmanipulated products available. It is plausible that the absence of any other products would increase the effect of the intervention, given less potential for its effect to be diluted.
- Summary risk of bias. Effect sizes for lower (versus higher) proximity were larger for studies at high risk of bias, versus both low risk of bias and those where there were some concerns.

- Socioeconomic context. Effect sizes for lower (versus higher) proximity were larger when deprivation status was low, than among studies with samples that were both high and low in deprivation.

At Stage 3, we examined intervention characteristics as covariates, and the following single variable was significantly associated with the intervention effect.

- Absolute difference in proximity. Effect sizes for lower (versus higher) proximity were larger the farther away the product was placed relative to a comparator. Increasing distance resulting in an increased intervention effect is consistent with the theory underlying why the intervention might work.

At Stage 4, we examined participant characteristics as covariates, and found no variables to be associated with the intervention effect.

In summary, meta-regression analyses indicated that the intervention effect was greater under four conditions: the farther away the product was placed relative to a comparator; when only the targeted product(s) (as opposed to a wider range) was available; when participants were of low (versus both high and low) deprivation status; and when the study was at high risk of bias. The results of this analysis should be interpreted with caution because the numbers of data points available for each included explanatory variable only barely exceeded the prespecified minimum level for inclusion (i.e. available data from a minimum of 10 studies). Furthermore, the associations cannot be interpreted as causal, 
and some of the study characteristics were correlated with each other. For example, all of the studies assessed as at high risk/ some concerns of bias were in low deprivation contexts (whilst all studies with samples that were both high and low in deprivation were at low risk of bias); and studies where there were other unmanipulated products available tended to be those in which the absolute difference in proximity was greater.

GRADE assessment applied to the analysis that excluded outliers indicated that the evidence for this outcome is of low certainty, meaning that current evidence provides some indication of the likely effect, but the likelihood that the actual effect will be substantially different is high. We reached this judgement through consideration of the following criteria. We downgraded the current evidence by one level (i.e. serious limitations) due to study limitations, as we judged the majority of study-level estimates of this effect to have significant concerns related to risk of bias. We did not downgrade the evidence for imprecision because even the lower $\mathrm{Cl}$ value indicated a small-to-moderate intervention effect, and the number of participants (sample size) incorporated into this meta-analysis was substantial and exceeded the optimal information size. We did not downgrade the evidence for inconsistency (as although heterogeneity was substantial, effect sizes were in a consistent direction with reasonable overlap of Cls) or indirectness. We downgraded the evidence by one level for publication bias because formal assessment of the degree of asymmetry present in a funnel plot suggested its presence.

Our prespecified sensitivity analyses concerning imputed data meant the removal of data from Painter 2002 and Wansink 2006 from the primary analysis. This did not alter the results or interpretation, with point estimates and $95 \% \mathrm{Cls}$ being similar to our primary meta-analysis (random-effects SMD $-0.66(95 \% \mathrm{Cl}$ -0.94 to $-0.37, \mathrm{P}<0.001)$; fixed-effect SMD $-0.46(95 \% \mathrm{Cl}-0.60$ to $-0.32, \mathrm{P}<0.001)$ ). We excluded data from two studies from the primary analysis for this outcome due to the intervention effect being confounded as part of multicomponent interventions (Cohen 2015; Greene 2017). In a prespecified sensitivity analysis, we reinstated these data, resulting in an analysis of 17 comparisons (2695 participants) identified from 14 food studies (Cohen 2015; Engell 1996 (S1); Engell 1996 (S2); Greene 2017; Hunter 2018 (S1); Hunter 2018 (S2); Hunter 2019; Langlet 2017; Maas 2012 (S1); Maas 2012 (S2); Musher-Eizenman 2010; Painter 2002; Privitera 2014; Wansink 2006). This did not alter the result or interpretation, with point estimates and $95 \% \mathrm{Cls}$ being similar to our primary metaanalysis (random-effects SMD $-0.56(95 \% \mathrm{Cl}-0.79$ to $-0.33, \mathrm{P}<$ $0.001)$; fixed-effect SMD $-0.44(95 \% \mathrm{Cl}-0.57$ to $-0.31, \mathrm{P}<0.001)$ ).

\section{Secondary outcomes}

Secondary outcomes, specified as selection and consumption outcomes relating to products not manipulated by the intervention, were rarely reported. This was principally because interventions manipulated all the available products, or because outcomes relating to non-manipulated products were not assessed. One availability study included secondary outcomes that met our criteria: Pechey 2019 reported that the intervention resulted in significantly less total energy $(-7.2 \%)$ being purchased, that is energy from all food categories, whether targeted by the intervention or not. Two proximity studies included pertinent secondary outcomes. Cohen 2015 reported no effect of the intervention on selection and consumption of non-manipulated main meals, and Kongsbak 2016 reported that the intervention resulted in less total energy being selected. Such limited reporting of these outcomes may be expected in laboratory studies featuring single or small numbers of products, but in this body of studies it was also rarely reported in field settings, which may also partly reflect the complexity of capturing and interpreting such data in complex real-world food environments. In sum, data on secondary outcomes were sparse and difficult to compare across studies due to differing study characteristics. There was, however, a small amount of evidence suggesting that compensatory behaviour - for example increased selection or consumption of non-manipulated products - did not occur. Furthermore, there was an absence of clear evidence suggesting that such compensatory behaviour did occur.

\section{DISCUSSION}

\section{Summary of main results}

The evidence in this review suggests that people select less of a targeted range or category of food(s) when fewer options of that food are available. Because the direction of comparisons within the analysis is interchangeable, they likewise select more when more options are available. However, there was greater uncertainty about the effect of such availability interventions on consumption. A separate body of evidence suggests that people consume less food when products are placed farther away (and likewise more when placed nearer), with a less certain effect on selection. Consequently, these interventions have the potential to have beneficial or harmful effects on health, depending on the characteristics of the food(s) that they focus upon.

The summary effect sizes derived from meta-analyses were considerable, suggesting potential impacts of between $17 \%$ and $36 \%$ on energy selected and consumed on an average snack occasion. Although such estimates are necessarily tentative due to limitations of the underlying data and the assumptions inherent to generating and applying them, if sustained effects of such magnitude were realised and extended to foods consumed over each day, reductions in daily energy intake would have the potential to make meaningful contributions to addressing major risk factors for non-communicable disease. For example, 10-year weight gain between 1999 and 2009 among adults in England (i.e. $9 \mathrm{~kg}$ at the 90th percentile) has been estimated to be equivalent to extra energy intake of around $24 \mathrm{kcal}$ per day over the same period (an amount equivalent to approximately $1.4 \%$ of average daily energy intake for UK adults) (Department of Health 2011). Any sustained reductions in daily energy intake exceeding this level are therefore likely to be effective in helping to prevent further weight gain in the population. Whilst these illustrations highlight the promise of these interventions, the sustainability of their effects has yet to be established, with the majority of studies in this review featuring short-term exposures to interventions, and because the certainty of the evidence varied and was assessed as low or very low for all outcomes, we can only have limited confidence in the effect estimates.

Importantly, the evidence base for this review was limited in quantity, often severely so, and was entirely absent for alcohol and tobacco products. Furthermore, evidence was sparse for secondary outcomes that could indicate the potential for unintended compensatory behaviour, although there was a small amount of evidence suggesting that this did not occur in response to the intervention and an absence of clear evidence suggesting that it did. Due to the lack of data, we were also unable 
to satisfactorily address the second objective of the review, concerning potential modifiers of the observed intervention effects (see Overall completeness and applicability of evidence).

\section{Overall completeness and applicability of evidence}

The completeness and applicability of the evidence was limited as a result of several characteristics of the evidence base. The synthesised evidence was collected from 24 included studies, with only six reporting availability interventions. In only one primary analysis (the effect of proximity on consumption) did the sample size exceed the optimal information size. The sample sizes weaken confidence that these studies enable us to address the first objective of this review. An additional impact of the limited quantity of evidence is that this reduces the representativeness of the evidence base across a myriad of possible study, intervention, and participant contexts. This means that the extent to which the results of the review apply to other contexts is uncertain. For example, the majority of studies were conducted in the USA, with no studies in LMICs, and participants were typically characterised by low social and material deprivation. These factors limit assessment of social differentiation in effects relevant to health equity. For example, while we have no reason to expect that mechanisms by which exposure to these interventions may influence behaviour will differ substantively and systematically between people living in HICs and those living in LMICs, a range of socio-cultural, economic, and contextual differences between these groups could plausibly modify effects. Studies are needed that focus on these contexts.

A further limitation regarding completeness concerns the setting of studies. The majority of included studies were conducted in laboratory settings - which even when relatively naturalistic in their design cannot convincingly replicate uncontrolled, realworld settings - although this limitation does apply differentially to availability and proximity interventions. For availability interventions, while there was a very small number of studies, the majority were conducted in field settings (namely schools, a childcare facility, worksite cafeterias, and supermarkets) and intervention exposures were over prolonged time periods. Despite this, there were insufficient data to draw meaningful conclusions specific to any one type of field setting (e.g. schools or supermarkets), given that contextual characteristics and participant behaviours are likely to differ substantially between these settings. Only four proximity studies were conducted in field settings. Laboratory-based studies of proximity interventions, as is typical for such contexts, usually assessed one-off exposures or repeated exposures over relatively short time periods, with correspondingly short-term outcomes. They also usually exposed participants to a single, or small range of, snack food intended for immediate consumption, limiting the degree to which such effects can be generalised to complex real-world food environments.

The most notable gap in this evidence base, however, was the absence of any eligible studies investigating effects of these interventions on selection or consumption of either alcohol or tobacco products. This finding is consistent with the small proportion of studies on alcohol and tobacco compared with food products, which we found in a large scoping review of interventions within physical micro-environments (Hollands 2013a; Hollands 2013b). This may be attributable to both a greater interest in, and more opportunities for, intervening on food products due to the broad range that are available, their ubiquity in multiple environments, and the necessity of their consumption. It may also reflect the proportion of research focused on reducing consumption of tobacco and alcohol, compared with food. Research on tobacco and alcohol has tended to consider treatments for the subgroup of the population addicted to those products, whereas studies in relation to food are more likely to take a whole-population approach. Furthermore, tobacco and alcohol are more highly regulated products in terms of where and how they can be sold, which may limit opportunities for research relative to food products.

Finally, due to the relatively small amount of available data, it was typically not possible to assess the impact of variations in characteristics between included studies on intervention effects. This meant that it was not possible to satisfactorily address the second objective of this review, namely to assess potential effect modifiers. It was only the effect of proximity on consumption that allowed meta-regression analyses - including only a small number of modifying variables - to be conducted. These analyses indicated that the intervention effect was greater: the farther away the product was placed relative to a comparator; when only the targeted product(s) (as opposed to a wider range) was available; when participants were of low (versus both high and low) deprivation status; and when the study was at high risk of bias. However, because the amount of data available only barely exceeded that which we set as the absolute minimum necessary, and due to their essentially observational nature, these findings should be given considerable caution. Before they can be meaningfully interpreted, they will require confirmation and replication in further research involving larger datasets, but ultimately may prove useful in evaluating, developing, and targeting interventions. Similarly, we were typically unable to examine whether potentially important associations were absent, in other words whether intervention effects were robust to variations in key intervention and participant characteristics. For example, while meta-regression analyses did not find that the proportion of female participants modified the intervention effect, which could add credence to the idea that altering environmental cues has the potential to impact behaviour across populations, we were unable to examine other key characteristics such as age or BMI.

\section{Quality of the evidence}

At the level of individual studies, the large majority of studies were subject to significant concerns about risk of bias, reflecting serious concerns about study limitations, compounded by unclear and incomplete reporting of study methods. Commonly, this derived from concerns about bias arising from the randomisation process, but we could not identify any obvious reason to prevent the implementation of unbiased procedures for random sequence generation and allocation concealment or reporting thereof. At the level of the evidence available for each outcome, we accounted for the significant concerns about risk of bias by downgrading each of these outcomes by one level within GRADE assessments. When also considering the full set of GRADE criteria for each outcome (detailed in Effects of interventions), we judged the evidence base to be of low certainty for the effect of availability on both selection and consumption (Summary of findings for the main comparison); low certainty for the effect of proximity on consumption; and very low certainty for the effect of proximity on selection (Summary of findings 2). In sum, this confers, at best, limited confidence 
in our estimated effects and necessitates due caution in their interpretation.

It is noted that Brian Wansink is an author on four of the studies included in the review (Greene 2017; Painter 2002; Wansink 2006; Wansink 2013a), two of which (Painter 2002; Wansink 2006) contributed data to our primary meta-analyses. This researcher has been subject to multiple retractions of his work due to academic misconduct (Munafò 2018). To date, none of the studies included in this review have been retracted, but should this occur, we will withdraw that study's data from updated meta-analyses conducted as part of a future update. Whilst these retractions introduce additional uncertainty regarding the veracity of other, unretracted studies he has authored, we chose not to report analyses that remove data from any studies authored by Wansink. This would assume that Wansink was principally responsible for the data reported in all studies for which he is an author - an unreasonable assumption without specific knowledge, and potentially unfair to co-authors. Relatedly, it could also set an unwelcome precedent for consistently excluding all data linked by co-authorship to an author who has had papers retracted due to academic misconduct.

Finally, we note that for 12 of the studies, potential commercial conflicts of interest were unclear, thus preventing us from eliminating the possibility that the review results could be biased in some way by interests of the study authors.

\section{Potential biases in the review process}

The potential for review author error and bias was reduced by involving at least two independent review authors in the selection of studies and the data extraction and study assessment processes. Whilst it remains possible that we failed to identify all relevant research for inclusion in the review, we used an extensive and highly sensitive search strategy involving a comprehensive range of databases and other sources, as well as backwards and forwards citation searches.

\section{Agreements and disagreements with other studies or reviews}

We are not aware of other systematic reviews that focus specifically on these interventions across all settings and product types and that also include a quantitative synthesis. Bucher 2016 conducted a systematic review with substantial content overlap with the 'proximity studies' within the current review. Although that review did not meta-analyse the identified studies, the conclusions were consistent with those of the current review, namely that manipulating the order of food products or their proximity can influence food choice. A wide range of other reviews include, but are not limited to, one or both of the target interventions within a scope determined by specific settings or product types. For example, Broers 2017 conducted a systematic review of a range of nudging interventions applied to fruit and vegetable choice, concluding that the largest effect was associated with interventions that altered the placement of products. Grech 2015 reviewed evidence for nutrition interventions applied to vending machines, concluding that there was evidence that altering availability was an effective means of improving the nutritional quality of products purchased. Finally, Cameron 2016 reviewed a broad range of evidence for supermarket-based interventions that included those changing product availability and placement, but the nature of the evidence meant that it was not possible to estimate effects of specific intervention strategies.

\section{AUTHORS' CONCLUSIONS}

\section{Implications for practice}

Given the complete absence of evidence for alcohol and tobacco products, the key implications of this review for public health policy and practice concern food products. Furthermore, we identified no evidence from low- and middle-income countries (LMICS), meaning that applicability to those contexts remains uncertain. Whilst, in practical terms, these interventions appear no less suitable for use in LMICs, due to, for example, their likely minimal (fixed and variable) costs, there is as yet insufficient evidence to judge their potential effectiveness or the feasibility of their implementation in such contexts.

This review suggests that policies and practices that alter the availability or proximity of food products could contribute to meaningful changes in the quantities of food that people select or consume, and could be used as part of a wider set of strategies to support healthier food consumption. With the exception of directly controlling availability and proximity, however, assessment of the effectiveness of possible intervention strategies that could achieve this was beyond the scope of this review. Furthermore, due to the limited quantity of current evidence and the low to very low certainty of this evidence, implications for practice are correspondingly tentative.

For interventions that alter availability, capitalising on these effects in a public health context could involve decreasing the absolute number of different options of a range or category of less-healthy food, or decreasing the relative number (proportion) of lesshealthy (to healthier) food options that are available within an environment. When the public health goal is to increase selection or consumption of healthier food(s), this could involve increasing the absolute number of different options of a range or category of healthier food, or increasing the relative number (proportion) of healthier (to less-healthy) options. In contrast to the proximity interventions, most of the small set of studies of availability were conducted in field settings, namely schools, a childcare facility, worksite cafeterias, and supermarkets, demonstrating that this is a strategy that is directly transferable to practice and can in theory be implemented in real-world settings. It therefore seems feasible that actions could be taken at a local level - by those who have direct responsibility for the characteristics of environments - whilst broader policy actions in at least some settings could ensure changes to availability. This could include mandating that the nutritional composition of certain ranges or categories of foods available within public-sector establishments like schools or hospitals meet specific nutritional criteria. For example, the Scottish Government and NHS Scotland have introduced the Healthcare Retail Standard (HRS) as a set of mandatory criteria for all retail outlets in all healthcare settings in Scotland, which include requiring at least $50 \%$ of products to be from a healthier range (Scottish Government 2015). In another example, as a result of pilot work, the availability of healthier options has been increased in vending machines in 105 National Health Service sites (Public Health England 2018b)

For interventions that alter proximity, capitalising on the observed effects could involve placing less-healthy food options in less 
immediately accessible positions relative to key environmental features (such as farther from entrances, checkouts, or walking or queueing routes), or placing healthier food options in more immediately accessible positions. In contrast to availability interventions, however, the evidence in this review derives principally from studies conducted in artificial laboratory settings. As such, while in principle such interventions would appear directly transferable to practice, their operationalisation and likely impact in real-world environments is less clear. Whilst it is challenging to identify practicable possibilities for intervention and to gauge the potential for higher-level policy actions that could impose subtle or context-specific changes to layouts of environments (such as changing the order of food presentation in cafeterias), actions could be initiated at the local level by those who have control of those environments. In addition, more comprehensive policy actions could encourage or mandate generalisable changes to environments, at least in certain contexts. For example, while checkout food policies in the UK are currently voluntary (Ejlerskov 2018a), the UK Government intends to consult on plans to ban through legislation the sale of unhealthy confectionery and snack foods at shop checkouts, end of aisles, and store entrances across the retail and out-of-home sectors. Such actions would result in these products being positioned in less accessible positions in shops (Department of Health and Social Care 2018). While they are as yet not directly informed by true experimental evidence, observational data from natural experiments suggest that supermarket polices to reduce less-healthy food at checkouts impact upon purchasing of those foods, namely small packages of confectionery and potato crisps (Ejlerskov 2018b).

There remains considerable uncertainty about the effects of these interventions when implemented under free-living conditions, and their longer-term sustainability. Their effectiveness will be subject to all the challenges and complexities of achieving effective implementation at scale and sustained over time. One such complexity is that scaling up interventions of this kind, increasing their geographic coverage and scope, would involve introducing them into a complex food environment, populated by a multitude of available food and drink products other than those that are being manipulated. This would raise the potential for compensatory consumption or substitution effects (see Implications for research), which the evidence assembled in this review does not sufficiently elucidate.

\section{Implications for research}

The key research implication of this review is that more highquality studies of both availability and proximity interventions in field settings are needed. Studies of these kinds of environmental interventions are viable in real-world settings, as evidenced by existing examples, although feasibility studies attest to the fact that they are highly challenging to implement and conduct (Hollands 2018; Pechey 2019; Vermeer 2009). Future studies should demonstrate improved rigour in conduct and reporting including pre-registration of study protocols that are publicly available, and with fundamental concerns about risk of bias addressed where possible in line with common standards. Furthermore, in order to systematically develop an understanding of the effect of manipulating environmental cues, studies should, where possible, manipulate environmental cues in isolation in interventions that are designed to be unconfounded in terms of their potentially active components (Allan 2017). These new studies should include those focusing on alcohol and tobacco products, for which there was a complete absence that met the inclusion criteria for this review (see Overall completeness and applicability of evidence). These interventions are in principle transferable to these products given the range of both different alcohol and tobacco (and recognised alternative) products and of environments in which these are selected and consumed.

A further key implication of this review is that future studies should be designed to better assess and report the potential impact of these interventions on selection and consumption both over time - with extended durations of intervention exposure and outcome measurement - and for other products available within a given environment but not targeted by a given intervention. This would increase our understanding of potential compensatory or substitution behaviour, which could concern shifts in selection or consumption to other untargeted food products or categories. For example, a measured reduction in chocolate consumption, in response to an intervention targeting chocolate, could be accompanied by an unmeasured and undesirable equivalent increase in sugary drink consumption. Additionally, an intervention could alter the temporal patterning of behaviour as a result of compensation. For example, a measured reduction in chocolate consumption at midday could be accompanied by an unmeasured equivalent or greater increase in chocolate consumption later in the day. More detailed examination is therefore necessary to enable better estimation of an intervention's overall impact on energy consumption and related outcomes. Given that the current evidence base predominantly comprises laboratory studies that are limited to manipulating and assessing behaviour in relation to single or very limited ranges of snack foods intended for immediate consumption, it is unable to adequately address this issue. Relevant evidence from field studies remains sparse.

When implemented in real-world settings, these interventions are likely to be applied to highly complex ranges of manipulated and non-manipulated products. Although in this review we did break down the availability and proximity interventions into more granular subtypes, these categorisations remain relatively nebulous and may not be satisfactorily descriptive. It will also therefore be important for the research community to develop a more detailed conceptual understanding, and related means of characterisation and reporting of these interventions. Building on work delineating broad types of interventions in physical micro-environments using the TIPPME typology (Hollands 2017a), developments in this area attempt to map the complex parameters of these interventions and provide a conceptual framework for better characterising them (Pechey under review). In future, such developments may enable a more nuanced analysis and understanding of the intervention characteristics that principally determine effectiveness.

These implications for research are derived from reviewing the current evidence base, which derives exclusively from studies conducted in high-income countries (HICs). It is feasible that they may apply similarly to research in LMICs as for HICs, in that, for example, there is no inherent reason why the nature of interventions and study designs need differ when testing these interventions within LMICs. However, the lack of experience in conducting studies of this kind in LMICs allows for the possibility that research issues specific to such settings may emerge as the evidence accumulates. 


\section{ACK N OWLEDGEMENTS}

Production of this review was funded by the National Institute for Health Research Policy Research Programme (Policy Research Unit in Behaviour and Health (PR-UN-0409-10109)). The funders had no role in the study design, data collection, analysis, or interpretation. The views expressed in this publication are those of the authors and not necessarily those of the funders,

the NHS, the National Institute for Health Research, the Department of Health and Social Care or its arm's length bodies, and other Government Departments. We would like to acknowledge the contributions of Julie Glanville (York Health
Economics Consortium, University of York, UK), who reviewed a draft of our MEDLINE search strategy; Claire Stansfield (EPPICentre, UCL Institute of Education, UK), who helped to develop our search strategy for the TRoPHI database; and Patrick Condron (Information Specialist, Cochrane Public Health Group), who reviewed further iterations of our MEDLINE and Embase strategies. We would like to thank Jodie Doyle (Managing Editor) and colleagues in the Cochrane Public Health Group. We would like to thank colleagues at the EPPI-Centre, UCL Institute of Education, UK, in particular James Thomas and Sergio Graziosi, for their help in developing technological solutions and managing those processes in order to implement a workable study identification process. 


\section{RE F E R E N C E S}

\section{References to studies included in this review}

\section{Cohen 2015 \{published data only\}}

Cohen JF, Richardson SA, Cluggish SA, Parker E, Catalano PJ, Rimm EB. Effects of choice architecture and chef-enhanced meals on the selection and consumption of healthier school foods: a randomized clinical trial. JAMA Pediatrics 2015;169:431-437.

\section{Engell 1996 (S1) \{published data only\}}

Engell D, Kramer M, Malafi T, Salomon M, Lesher L. Effects of effort and social modeling on drinking in humans. Appetite 1996;26:129-138.

\section{Engell 1996 (S2) \{published data only\}}

Engell D, Kramer M, Malafi T, Salomon M, Lesher L. Effects of effort and social modeling on drinking in humans. Appetite 1996;26:129-138.

\section{Fiske 2004 \{published data only\}}

Fiske A, Cullen KW. Effects of promotional materials on vending sales of low-fat items in teachers' lounges. Journal of the American Dietetic Association 2004;104:90-93.

\section{Foster 2014 \{published data only\}}

Foster GD, Karpyn A, Wojtanowski AC, Davis E, Weiss S, Brensinger $\mathrm{C}$, et al. Placement and promotion strategies to increase sales of healthier products in supermarkets in lowincome, ethnically diverse neighborhoods: a randomized controlled trial. American Journal of Clinical Nutrition 2014;9:1359-68.

\section{Greene 2017 \{published data only\}}

Greene KN, Gabrielyan G, Just DR, Wansink B. Fruit-promoting smarter lunchrooms interventions: results from a cluster RCT. American Journal of Preventive Medicine 2017;52(4):451-458.

\section{Hunter 2018 (S1) \{published data only\}}

* Hunter JA, Hollands GJ, Couturier DL, Marteau TM. Effect of snack-food proximity on intake in general population samples with higher and lower cognitive resource. Appetite 2018;121:337-347.

Hunter JA, Hollands GJ, Couturier DL, Marteau TM. Impact of altering proximity on snack food intake in individuals with high and low executive function: study protocol. BMC Public Health 2016;16:504.

\section{Hunter 2018 (S2) \{published data only\}}

* Hunter JA, Hollands GJ, Couturier DL, Marteau TM. Effect of snack-food proximity on intake in general population samples with higher and lower cognitive resource. Appetite 2018;121:337-347.

Hunter JA, Hollands GJ, Couturier DL, Marteau TM. Impact of altering proximity on snack food intake in individuals with high and low executive function: study protocol. BMC Public Health 2016;16:504.

\section{Hunter 2019 \{published data only\}}

Hunter JA, Hollands GJ, Pilling M, Marteau TM. Impact of proximity of healthier versus less healthy foods on selection: a lab-based experiment. Appetite 2019;133:147-155.

\section{Kocken 2012 \{published data only\}}

Kocken PL, Eeuwijk J, Van Kesteren NM, Dusseldorp E, Buijs G, Bassa-Dafesh Z, et al. Promoting the purchase of low-calorie foods from school vending machines: a cluster-randomized controlled study. Journal of School Health 2012;82:115-122.

\section{Kongsbak 2016 \{published data only\}}

Kongsbak I, Skov LR, Nielsen BK, Ahlmann FK, Schaldemose H, Atkinson L, et al. Increasing fruit and vegetable intake among male university students in an ad libitum buffet setting: a choice architectural nudge intervention. Food Quality and Preference 2016;49:183-188.

\section{Langlet 2017 \{published data only\}}

Langlet B, Fagerberg P, Glossner A, loakimidis I. Objective quantification of the food proximity effect on grapes, chocolate and cracker consumption in a Swedish high school. A temporal analysis. PLOS ONE 2017;12(8):e0182172.

\section{Maas 2012 (S1) \{published data only\}}

Maas J, De Ridder DTD, Vet De E. Do distant foods decrease intake? The effect of food accessibility on eating behaviour. Psychology \& Health 2010;25(SI):55.

* Maas J, de Ridder DT, de Vet E, de Wit JB. Do distant foods decrease intake? The effect of food accessibility on consumption. Psychology \& Health 2012;27:59-73.

Maas 2012 (S2) \{published data only\}

Maas J, De Ridder DTD, Vet De E. Do distant foods decrease intake? The effect of food accessibility on eating behaviour. Psychology \& Health 2010;25(SI):55.

* Maas J, de Ridder DT, de Vet E, de Wit JB. Do distant foods decrease intake? The effect of food accessibility on consumption. Psychology \& Health 2012;27:59-73.

\section{Musher-Eizenman 2010 \{published data only\}}

Musher-Eizenman DR, Young KM, Laurene K, Galliger C, Hauser J, Wagner Oehlhof M. Children's sensitivity to external food cues: how distance to serving bowl influences children's consumption. Health Education \& Behavior 2010;37:186-192.

\section{Painter 2002 \{published data only\}}

Painter JE, Wansink B, Hieggelke JB. How visibility and convenience influence candy consumption. Appetite 2002;38:237-238.

\section{Pechey 2019 \{published data only\}}

Pechey R, Cartwright E, Pilling M, Hollands GJ, Jebb SA, Vasiljevic M, et al. Impact of increasing the proportion of healthier foods available on energy purchased in worksite cafeterias: a stepped wedge randomized controlled pilot trial. Appetite 2019;133:286-296. 
Privitera 2012 (S1) \{published data only\}

Privitera GJ, Creary HE. Proximity and visibility of fruits and vegetables influence intake in a kitchen setting among college students. Environment and Behavior 2012;45:876-886.

\section{Privitera 2012 (S2) \{published data only\}}

Privitera GJ, Creary HE. Proximity and visibility of fruits and vegetables influence intake in a kitchen setting among college students. Environment and Behavior 2012;45:876-886.

\section{Privitera 2014 \{published data only\}}

Privitera GJ, Zuraikat FM. Proximity of foods in a competitive food environment influences consumption of a low calorie and a high calorie food. Appetite 2014;76:175-179.

Roe 2013 \{published data only\}

Roe LS, Meengs JS, Birch LL, Rolls BJ. Serving a variety of vegetables and fruit as a snack increased intake in preschool children. American Journal of Clinical Nutrition 2013;98:693-699.

\section{Stubbs 2001 \{published data only\}}

Stubbs RJ, Johnstone AM, Mazlan N, Mbaiwa SE, Ferris S. Effect of altering the variety of sensorially distinct foods, of the same macronutrient content, on food intake and body weight in men. European Journal of Clinical Nutrition 2001;55:19-28.

\section{Wansink 2006 \{published data only\}}

Wansink B, Painter JE, Lee Y-K. The office candy dish: proximity's influence on estimated and actual consumption. International Journal of Obesity 2006;30:871-875.

\section{Wansink 2013a \{published data only\}}

Wansink B, Hanks AS. Slim by design: serving healthy foods first in buffet lines improves overall meal selection. PLOS ONE 2013;8:e77055.

\section{References to studies excluded from this review}

\section{Alinia 2011 \{published data only\}}

Alinia S, Lassen AD, Krogholm KS, Christensen T, Hels OH, Tetens I. A workplace feasibility study of the effect of a minimal fruit intervention on fruit intake. Public Health Nutrition 2011;14:1382-1387.

\section{Altintzoglou 2015 \{published data only\}}

Altintzoglou T, Skuland A, Carlehog M, Sone I, Heide M, Honkanen P. Providing a food choice option increases children's liking of fish as part of a meal. Food Quality and Preference 2015;39:117-123.

\section{Anderson 2005 \{published data only\}}

Anderson AS, Porteous LE, Foster E, Higgins C, Stead M, Hetherington $\mathrm{M}$, et al. The impact of a school-based nutrition education intervention on dietary intake and cognitive and attitudinal variables relating to fruits and vegetables. Public Health Nutrition 2005;8:650-656.

\section{Angelopoulos 2009 \{published data only\}}

Angelopoulos PD, Milionis HJ, Grammatikaki E, Moschonis G, Manios Y. Changes in BMI and blood pressure after a school based intervention: The CHILDREN study. European Journal of Public Health 2009;19:319-325.

Ayala 2013 \{published data only\}

Ayala GX, Baquero B, Laraia BA, Ji M, Linnan L. Efficacy of a store-based environmental change intervention compared with a delayed treatment control condition on store customers' intake of fruits and vegetables. Public Health Nutrition 2013;16:1954-1960.

Ayala 2015 \{published data only\}

Ayala GX, Baquero B, Pickrel JL, Mayer J, Belch G, Rock CL, et al. A store-based intervention to increase fruit and vegetable consumption: the El Valor de Nuestra Salud cluster randomized controlled trial. Contemporary Clinical Trials 2015;42:228-238.

Backman 2011 \{published data only\}

Backman D, Gonzaga G, Sugerman S, Francis D, Cook S. Effect of fresh fruit availability at worksites on the fruit and vegetable consumption of low-wage employees. Journal of Nutrition Education and Behavior 2011;43:S113-121.

Bartholomew 2006 \{published data only\}

Bartholomew JB, Jowers EM. Increasing frequency of lowerfat entrees offered at school lunch: an environmental change strategy to increase healthful selections. Journal of the American Dietetic Association 2006;106:248-252.

\section{Beets 2014 \{published data only\}}

Beets MW, Tilley F, Kyryliuk R, Weaver RG, Moore JB, TurnerMcGrievy G. Children select unhealthy choices when given a choice among snack offerings. Journal of the Academy of Nutrition and Dietetics 2014;114:1440-1446.

\section{Bere 2006 \{published data only\}}

Bere E, Veierød MB, Bjelland M, Klepp KI. Free school fruit - sustained effect 1 year later. Health Education Research 2006;21:268-275

\section{Bergamaschi 2016 \{published data only\}}

Bergamaschi V, Olsen A, Laureati M, Zangenberg S, Pagliarini E, Bredie WLP. Variety in snack servings as determinant for acceptance in school children. Appetite 2016;96:628-635.

\section{Bonsergent 2013 \{published data only\}}

Bonsergent E, Agrinier N, Thilly N, Tessier S, Legrand K, Lecomte $\mathrm{E}$, et al. Overweight and obesity prevention for adolescents: a cluster randomized controlled trial in a school setting. American Journal of Preventive Medicine 2013;44:30-39.

\section{Bonvecchio-Arenas 2010 \{published data only\}}

Bonvecchio-Arenas A, Theodore FL, Hernandez-Cordero S, Campirano-Nunez F, Islas AL, Safdie M, et al. The school as an opportunity for obesity prevention: an experience from the Mexican school system. Revista Espanola De Nutricion Comunitaria [Spanish Journal of Community Nutrition] 2010;16:13-16. 
Bucher 2011 \{published data only\}

Bucher T, van der Horst K, Siegrist M. Improvement of meal composition by vegetable variety. Public Health Nutrition 2011;14:1357-1363.

\section{Bucher 2014 \{published data only\}}

Bucher T, Siegrist M, van der Horst K. Vegetable variety: an effective strategy to increase vegetable choice in children. Public Health Nutrition 2014;17:1232-1236.

\section{Burns 2015 \{published data only\}}

Burns RJ, Rothman AJ. Offering variety: a subtle manipulation to promote healthy food choice throughout the day. Health Psychology 2015;34:566-570.

\section{Butler 1996 \{published data only\}}

Butler BA, Wing RR, Shiffman S. Effect of the number of highfat and low-fat cues on food choice. International Journal of Behavioral Medicine 1996;3:42-54.

\section{Caldwell 2009 \{published data only\}}

Caldwell EM, Miller Kobayashi M, DuBow WM, Wytinck SM. Perceived access to fruits and vegetables associated with increased consumption. Public Health Nutrition 2009;12:1743-1750.

\section{Carroll 2018 \{published data only\}}

Carroll KA, Samek A, Zepeda L. Food bundling as a health nudge: investigating consumer fruit and vegetable selection using behavioral economics. Appetite 2018;121:237-248.

\section{Chandon 2002 \{published data only\}}

Chandon P, Wansink B. When are stockpiled products consumed faster? A convenience-salience framework of postpurchase consumption incidence and quantity. Journal of Marketing Research 2002;39:321-335.

\section{Cohen 2014 \{published data only\}}

Cohen JF, Rimm EB, Austin SB, Hyatt RR, Kraak VI, Economos $C D$. A food service intervention improves whole grain access at lunch in rural elementary schools. Journal of School Health 2012;84:212-219.

\section{Coleman 2012 \{published data only\}}

Coleman KJ, Shordon M, Caparosa SL, Pomichowski ME, Dzewaltowski DA. The healthy options for nutrition environments in schools (Healthy ONES) group randomized trial: using implementation models to change nutrition policy and environments in low income schools. International Journal of Behavioral Nutrition and Physical Activity 2012;9:80.

Dayan 2011 \{published data only\}

Dayan E, Bar-Hillel M. Nudge to nobesity II: Menu positions influence food orders. Judgment and Decision Making 2011;6:333-342.

\section{De Wild 2015 \{published data only\}}

de Wild VWT, de Graaf C, Boshuizen HC, Jager G. Influence of choice on vegetable intake in children: an in-home study. Appetite 2015;91:1-6.

\section{Divert 2015 \{published data only\}}

Divert C, Laghmaoui R, Crema C, Issanchou S, Wymelbeke VV, Sulmont-Rossé C. Improving meal context in nursing homes. Impact of four strategies on food intake and meal pleasure. Appetite 2015;84:139-147.

\section{Dominguez 2013 \{published data only\}}

Dominguez PR, Gamiz F, Gil M, Moreno H, Zamora RM, Gallo M, de Brugada I. Providing choice increases children's vegetable intake. Food Quality and Preference 2013;30:108-113.

Elsbernd 2016 \{published data only\}

Elsbernd SL, Reicks MM, Mann TL, Redden JP, Mykerezi E, Vickers ZM. Serving vegetables first: a strategy to increase vegetable consumption in elementary school cafeterias. Appetite 2016;96:111-115.

Epstein 2009 \{published data only\}

Epstein LH, Robinson JL, Temple JL, Roemmich JN, Marusewski AL, Nadbrzuch RL. Variety influences habituation of motivated behavior for food and energy intake in children. American Journal of Clinical Nutrition 2009;89:746-754.

Epstein 2013 \{published data only\}

Epstein LH, Fletcher KD, O'Neill J, Roemmich JN, Raynor H, Bouton ME. Food characteristics, long-term habituation and energy intake: laboratory and field studies. Appetite 2013;60:40-50.

Epstein 2015 \{published data only\}

Epstein LH, Kilanowski C, Paluch RA, Raynor H, Daniel TO. Reducing variety enhances effectiveness of familybased treatment for pediatric obesity. Eating Behaviors 2015;17:140-143.

Eriksen 2003 \{published data only\}

Eriksen K, Haraldsdóttir J, Pederson R, Flyger HV. Effect of a fruit and vegetable subscription in Danish schools. Public Health Nutrition 2003;6:57-63.

\section{Fisher 1999 \{published data only\}}

Fisher JO, Birch LL. Restricting access to palatable foods affects children's behavioral response, food selection, and intake. American Journal of Clinical Nutrition 1999;69:1264-1272.

\section{French 2004 \{published data only\}}

French SA, Story M, Fulkerson JA, Hannan P. An Environmental Intervention to Promote Lower-Fat Food Choices in Secondary Schools: Outcomes of the TACOS Study. American Journal of Public Health 2004;94:1507-1512.

French 2005a \{published data only\}

French SA. School-based programmes to promote and increase availability of lower fat foods increases sales of lower fat foods to students. Evidence-Based Healthcare and Public Health 2005;9:141-142.

French 2005b \{published data only\}

French SA. Public health strategies for dietary change: schools and workplaces. Journal of Nutrition 2005;135:910-912. 


\section{French 2010a \{published data only\}}

French SA, Hannan PJ, Harnack LJ, Mitchell NR, Toomey TL, Gerlach A. Pricing and availability intervention in vending machines at four bus garages. Journal of Occupational and Environmental Medicine 2010;52:S29.

\section{French 2010b \{published data only\}}

French SA, Harnack LJ, Hannan PJ, Mitchell NR, Gerlach AF, Toomey TL. Worksite environment intervention to prevent obesity among metropolitan transit workers. Preventive Medicine 2010;50:180-185.

\section{Giles 2012 \{published data only\}}

Giles CM, Kenney EL, Gortmaker SL, Lee RM, Thayer JC, Mont-Ferguson $\mathrm{H}$, et al. Increasing water availability during afterschool snack: evidence, strategies, and partnerships from a group randomized trial. American Journal of Preventive Medicine 2012;43:S136-142.

\section{Gillis 2009 \{published data only\}}

Gillis B, Mobley C, Stadler DD, Hartstein J, Virus A, Volpe SL, et al. HEALTHY Study Group. Rationale, design and methods of the HEALTHY study nutrition intervention component. International Journal of Obesity 2009;33:S29-36.

\section{Gittelsohn 2013 \{published data only\}}

Gittelsohn J, Kim EM, He S, Pardilla M. A food store-based environmental intervention is associated with reduced $\mathrm{BMI}$ and improved psychosocial factors and food-related behaviors on the Navajo nation. Journal of Nutrition 2013;143:1494-1500.

\section{Hanks 2012 \{published data only\}}

Hanks AS, Just DR, Smith LE, Wansink B. Healthy convenience: nudging students toward healthier choices in the lunchroom. Journal of Public Health 2012;34:370-376.

\section{Harnack 2012 \{published data only\}}

Harnack LJ, Oakes JM, French SA, Rydell SA, Farah FM, Taylor GL. Results from an experimental trial at a Head Start center to evaluate two meal service approaches to increase fruit and vegetable intake of preschool aged children. International Journal of Behavioral Nutrition and Physical Activity 2012;9:51.

\section{Haws 2013 \{published data only\}}

Haws KL, Redden JP. In control of variety. High self-control reduces the effect of variety on food consumption. Appetite 2013;69:196-203.

\section{Hoerr 1993 \{published data only\}}

Hoerr SM, Louden VA. Can nutrition information increase sales of healthful vended snacks?. Journal of School Health 1993;63:386-390.

\section{Kahn 2004 \{published data only\}}

Kahn BE, Wansink B. The influence of assortment structure on perceived variety and consumption quantities. Journal of Consumer Research 2004;30:519-533.
Keller 2015 \{published data only\}

Keller C, Markert F, Bucher T. Nudging product choices: the effect of position change on snack bar choice. Food Quality and Preference 2015;41:41-43.

\section{Kim 2012 \{published data only\}}

Kim K, Hong SA, Yun SH, Ryou HJ, Lee SS, Kim MK. The effect of a healthy school tuck shop program on the access of students to healthy foods. Nutrition Research and Practice 2012;6:138-145.

\section{Kimathi 2009 \{published data only\}}

Kimathi AN, Gregoire MB, Dowling RA, Stone MK. A healthful options food station can improve satisfaction and generate gross profit in a worksite cafeteria. Journal of the American Dietetic Association 2009;109:914-917.

\section{Kingsland 2011 \{published data only\}}

Kingsland M, Wolfenden L, Rowland BC, Tindall J, Gillham KE, McElduff $P$, et al. A cluster randomised controlled trial of a comprehensive accreditation intervention to reduce alcohol consumption at community sports clubs: study protocol. BMJ Open 2011;1:e000328.

\section{Kocken 2015 \{published data only\}}

Kocken PL, van Kesteren NM, Buijs G, Snel J, Dusseldorp E. Students' beliefs and behaviour regarding low-calorie beverages, sweets or snacks: are they affected by lessons on healthy food and by changes to school vending machines?. Public Health Nutrition 2015;18:1545-1553.

\section{Kovalskys 2010 \{published data only\}}

Kovalskys I, Herscovici C, De Gregorio V, Ruben MJ, Schvartz M, Tron M, et al. A school based obesity prevention programmed in Rosario, Argentina: impact on intake changes of healthy and unhealthy foods. Obesity Reviews 2010;11:469.

Kroese 2016 \{published data only\}

Kroese FM, Marchiori DR, de Ridder DT. Nudging healthy food choices: a field experiment at the train station. Journal of Public Health 2016;38:e133-7.

\section{Lachat 2009 \{published data only\}}

Lachat CK, Verstraeten R, De Meulenaer B, Menten J, Huybregts LF, Van Camp J, et al. Availability of free fruits and vegetables at canteen lunch improves lunch and daily nutritional profiles: a randomised controlled trial. British Journal of Nutrition 2009;102:1030-1037.

\section{Leak 2014 \{published data only\}}

Leak T, Swenson A, Vickers Z, Reicks M. Feasibility and effectiveness of in-home behavioral economic strategies to increase vegetable intake among low-income children. FASEB Journal 2014;28:808.18.

\section{Lee 2014 \{published data only\}}

Lee $\mathrm{SH}$. Changing the food environment in Baltimore city: impact of an intervention to improve carry-outs in low-income neighborhoods. Dissertation Abstracts International: Section B: The Sciences and Engineering (Doctoral dissertation; Johns Hopkins University, Baltimore, Maryland) 2014; Vol. 74. 
Lee-Kwan 2013 \{published data only\}

Lee-Kwan SH, Goedkoop S, Yong R, Batorsky B, Hoffman V, Jeffries J, et al. Development and implementation of the Baltimore healthy carry-outs feasibility trial: process evaluation results. BMC Public Health 2013;13:638.

Linde 2012 \{published data only\}

Linde JA, Nygaard KE, MacLehose RF, Mitchell NR, Harnack LJ, Cousins JM, et al. HealthWorks: results of a multi-component group-randomized worksite environmental intervention trial for weight gain prevention. International Journal of Behavioral Nutrition and Physical Activity 2012;9:14.

\section{Loughridge 2005 \{published data only\}}

Loughridge JL, Barratt J. Does the provision of cooled filtered water in secondary school cafeterias increase water drinking and decrease the purchase of soft drinks?. Journal of Human Nutrition and Dietetics 2005;18:281-286.

\section{Lytle 2006 \{published data only\}}

Lytle LA, Kubik MY, Perry C, Story M, Birnbaum AS, Murray DM. Influencing healthful food choices in school and home environments: results from the TEENS study. Preventive Medicine 2006;43:8-13.

\section{Marcus 2009 \{published data only\}}

Marcus C, Nyberg G, Nordenfelt A, Karpmyr M, Kowalski J, Ekelund U. A 4-year, cluster-randomized, controlled childhood obesity prevention study: STOPP. International Journal of Obesity 2009;33:408-417.

\section{Martinez-Donate 2015 \{published data only\}}

Martínez-Donate AP, Riggall AJ, Meinen AM, Malecki K, Escaron AL, Hall B, et al. Evaluation of a pilot healthy eating intervention in restaurants and food stores of a rural community: a randomized community trial. BMC Public Health 2015;15:136.

\section{Meengs 2012 \{published data only\}}

Meengs JS, Roe LS, Rolls BJ. Vegetable variety: an effective strategy to increase vegetable intake in adults. Journal of the Academy of Nutrition and Dietetics 2012;112:1211-1215.

\section{Mennella 2008 \{published data only\}}

Mennella JA, Nicklaus S, Jagolino AL, Yourshaw LM. Variety is the spice of life: strategies for promoting fruit and vegetable acceptance during infancy. Physiology and Behavior 2008;94:29-38

\section{Meyers 1980 \{published data only\}}

Meyers AW, Stunkard AJ, Coll M. Food accessibility and food choice. A test of Schachter's externality hypothesis. Archives of General Psychiatry 1980;37:1133-1135.

\section{Moore 2008 \{published data only\}}

Moore L, Tapper K. The impact of school fruit tuck shops and school food policies on children's fruit consumption: a cluster randomised trial of schools in deprived areas. Journal of Epidemiology and Community Health 2008;62:926-931.

\section{Muckelbauer 2009 \{published data only\}}

Muckelbauer R, Libuda L, Clausen K, Reinehr T, Kersting M. A simple dietary intervention in the school setting decreased incidence of overweight in children. Obesity Facts 2009;2:282-285.

\section{Muckelbauer 2009b \{published data only\}}

Muckelbauer R, Libuda L, Clausen K, Toschke AM, Reinehr T, Kersting M. Promotion and provision of drinking water in schools for overweight prevention: randomized, controlled cluster trial. Pediatrics 2009;123:e661-7.

\section{Norton 2006 \{published data only\}}

Norton GN, Anderson AS, Hetherington MM. Volume and variety: relative effects on food intake. Physiology and Behavior 2006;87:714-722.

\section{Novotny 2011 \{published data only\}}

Novotny R, Vijayadeva V, Ramirez V, Lee SK, Davison N, Gittelsohn J. Development and implementation of a food system intervention to prevent childhood obesity in rural Hawai'i. Hawaii Medical Journal 2011;70:42-46.

O'Connell 2012 \{published data only\}

O'Connell ML, Henderson KE, Luedicke J, Schwartz MB. Repeated exposure in a natural setting: a preschool intervention to increase vegetable consumption. Journal of the Academy of Nutrition and Dietetics 2012;112:230-234.

Parker 2001 \{published data only\}

Parker L, Fox A. The Peterborough Schools Nutrition Project: a multiple intervention programme to improve schoolbased eating in secondary schools. Public Health Nutrition 2001;4:1221-1228.

\section{Patel 2011 \{published data only\}}

Patel AI, Bogart LM, Elliott MN, Lamb S, Uyeda KE, HawesDawson J, et al. Increasing the availability and consumption of drinking water in middle schools: a pilot study. Preventing Chronic Disease 2011;8:A60.

\section{Perez-Morales 2011 \{published data only\}}

Pérez-Morales ME, Bacardí-Gascón M, Jiménez-Cruz A. Longterm randomized school-based intervention: effect on obesity and lifestyles in Mexico. Obesity Reviews 2011;12:74.

\section{Perry 2004 \{published data only\}}

Perry CL, Bishop DB, Taylor GL, Davis M, Story M, Gray C, et al. A randomized school trial of environmental strategies to encourage fruit and vegetable consumption among children. Health Education \& Behavior 2004;31:65-76.

\section{Rausch 2013 \{published data only\}}

Rausch Herscovici C, Kovalskys I, De Gregorio MJ. Gender differences and a school-based obesity prevention program in Argentina: a randomized trial. Revista Panamericana de Salud Publica 2013;34:75-82.

\section{Raynor 2006 \{published data only\}}

Raynor HA, Wing RR. Effect of limiting snack food variety across days on hedonics and consumption. Appetite 2006;46:168-176. 


\section{Raynor 2012a \{published data only\}}

Raynor HA. Can limiting dietary variety assist with reducing energy intake and weight loss?. Physiology \& Behavior 2012;106:356-361.

\section{Raynor 2012b \{published data only\}}

Raynor HA, Osterholt KM. Greater variety of fruit served in a four-course snack increases fruit consumption. Appetite 2012;59:662-667.

\section{Raynor 2012c \{published data only\}}

Raynor HA, Steeves EA, Hecht J, Fava JL, Wing RR. Limiting variety in non-nutrient-dense, energy-dense foods during a lifestyle intervention: a randomized controlled trial. American Journal of Clinical Nutrition 2012:95:1305-1314.

\section{Redden 2015 \{published data only\}}

Redden JP, Mann T, Vickers Z, Mykerezi E, Reicks M, Elsbernd S. Serving first in isolation increases vegetable intake among elementary schoolchildren. PLOS ONE 2015;10:e0121283.

\section{Romero 2016 \{published data only\}}

Romero M, Biswas D. Healthy-left, unhealthy-right: can displaying healthy items to the left (versus right) of unhealthy items nudge healthier choices?. Journal of Consumer Research 2016;43(1):103-112.

\section{Rozin 2011 \{published data only\}}

Rozin P, Scott S, Dingley M, Urbanek JK, Jiang H, Kaltenbach M. Nudge to nobesity I: Minor changes in accessibility decrease food intake. Judgment and Decision Making 2011;6:323-332.

Sallis 2003 \{published data only\}

Sallis JF, McKenzie TL, Conway TL, Elder JP, Prochaska JJ, Brown M, et al. Environmental interventions for eating and physical activity - a randomized controlled trial in middle schools. American Journal of Preventive Medicine 2003;24:209-217.

\section{Scherr 2013 \{published data only\}}

Scherr RE, Linnell J, Smith M, Nicholson Y, Spezzano T, Bergman J, et al. The shaping healthy choices program: a multicomponent, school-based approach to improve children's nutrition and health behaviors while supporting regional agriculture. FASEB Journal 2013;27:623.6.

\section{Scherr 2014 \{published data only\}}

Scherr R, Linnell J, Smith M, Nicholson Y, Spezzano T, Bergman J, et al. The Shaping Healthy Choices Program results in improved nutrition and health-related outcomes. FASEB Journal 2014;28:273.6.

\section{Schwartz 2009 \{published data only\}}

Schwartz MB, Novak SA, Fiore SS. The impact of removing snacks of low nutritional value from middle schools. Health Education and Behavior 2009;36:999-1011.

\section{Shin 2015 \{published data only\}}

Shin A, Surkan PJ, Coutinho AJ, Suratkar SR, Campbell RK, Rowan M, et al. Impact of Baltimore healthy eating zones: an environmental intervention to improve diet among African American youth. Health Education \& Behavior 2015;42:97S-105S.

Sigurdsson 2011 \{published data only\}

Sigurdsson V, Larsen NM, Gunnarsson D. An in-store experimental analysis of consumers' selection of fruits and vegetables. Service Industries Journal 2011;31:2587-2602.

\section{Song 2008 \{published data only\}}

Song HJ. Development, implementation and evaluation of an urban food store intervention for Korean corner stores in Baltimore city. Dissertation Abstracts International: Section B: The Sciences and Engineering 2008;69:2277.

\section{Stea 2009 \{published data only\}}

Stea TH, Uglem S, Wandel M, Mansoor MA, Frølich W. Association between folate intake from different food sources in Norway and homocysteine status in a dietary intervention among young male adults. British Journal of Nutrition 2009;102:899-906.

\section{Steenhuis 2004 \{published data only\}}

Steenhuis I, Van Assema P, Van Breukelen G, Glanz K, Kok G, De Vries $\mathrm{H}$. The impact of educational and environmental interventions in Dutch worksite cafeterias. Health Promotion International 2004;19:335-343.

\section{Steeves 2015 \{published data only\}}

Steeves EA, Penniston E, Rowan M, Steeves J, Gittelsohn J. A rural small food store pilot intervention creates trends toward improved healthy food availability. Journal of Hunger \& Environmental Nutrition 2015;10:259-270.

\section{Steyn 2015 \{published data only\}}

Steyn NP, de Villiers A, Gwebushe N, Draper CE, Hill J, de Waal M, et al. Did HealthKick, a randomised controlled trial primary school nutrition intervention improve dietary quality of children in low-income settings in South Africa?. BMC Public Health 2015;15:948.

\section{Temple 2008 \{published data only\}}

Temple JL, Giacomelli AM, Roemmich JN, Epstein LH. Dietary variety impairs habituation in children. Health Psychology 2008;27:S10-9.

\section{Te Velde 2008 \{published data only\}}

Te Velde SJ, Brug J, Wind M, Hildonen C, Bjelland M, PérezRodrigo $C$, et al. Effects of a comprehensive fruit- and vegetablepromoting school-based intervention in three European countries: the Pro Children Study. British Journal of Nutrition 2008;10:893-903.

\section{Thorndike 2016 \{published data only\}}

Thorndike AN, Bright OM, Dimond MA, Fishman R, Levy DE. Choice architecture increases WIC fruit and vegetable purchases in a Latino community: randomized, controlled corner store intervention. Circulation 2016;133:A03.

Thorndike 2017 \{published data only\}

Thorndike AN, Bright OM, Dimond MA, Fishman R, Levy DE. Choice architecture to promote fruit and vegetable purchases 
by families participating in the Special Supplemental Program for Women, Infants, and Children (WIC): randomized corner store pilot study. Public Health Nutrition 2017;20(7):1297-1305.

\section{Toft 2017 \{published data only\}}

Toft U, Winkler LL, Mikkelsen BE, Bloch P, Glümer C. Discounts on fruit and vegetables combined with a space management intervention increased sales in supermarkets. European Journal of Clinical Nutrition 2017;71(4):476-480.

Uglem 2013 \{published data only\}

Uglem S, Stea TH, Kjøllesdal MKR, Frølich W, Wandel M. A nutrition intervention with a main focus on vegetables and bread consumption among young men in the Norwegian National Guard. Food \& Nutrition Research 2013;57:10.3402.

Uglem 2014 \{published data only\}

Uglem S, Råberg Kjøllesdal MK, Frølich W, Wandel M. Effect of a nutrition intervention on intake of vegetables, fruits, and semi whole grain bread among low and high consumers in the Norwegian National Guard. Military Medicine 2014;179:1013-1020.

van Herpen 2014 \{published data only\}

van Herpen E, Pieters R, Zeelenberg M. When less sells more or less: the scarcity principle in wine choice. Food Quality and Preference 2014;36:153-160.

van Kleef 2012 \{published data only\}

van Kleef E, Otten K, van Trijp HC. Healthy snacks at the checkout counter: a lab and field study on the impact of shelf arrangement and assortment structure on consumer choices. BMC Public Health 2012;12:1072.

\section{Van Lippevelde 2012 \{published data only\}}

Van Lippevelde W, van Stralen M, Verloigne M, De Bourdeaudhuij I, Deforche B, Brug J, Maes L, Haerens L. Mediating effects of home-related factors on fat intake from snacks in a school-based nutrition intervention among adolescents. Health Education Research 2012;27:36-45.

\section{Visscher 2010 \{published data only\}}

Visscher TL, van Hal WC, Blokdijk L, Seidell JC, Renders CM, Bemelmans WJ. Feasibility and impact of placing water coolers on sales of sugar-sweetened beverages in Dutch secondary school canteens. Obesity Facts 2010;3:109-115.

\section{Wansink 2011 \{published data only\}}

Wansink B, Just DR, Smith LE, Wallace CE. Lunch line redesign: making school lunchrooms smarter. FASEB Journal 2011;25:342.8.

\section{Wansink 2013b \{published data only\}}

Wansink B, Shimizu M, Brumberg A. Association of nutrientdense snack combinations with calories and vegetable intake. Pediatrics 2013;131:22-29.

\section{Wijnhoven 2015 \{published data only\}}

Wijnhoven HA, van der Meij BS, Visser M. Variety within a cooked meal increases meal energy intake in older women with a poor appetite. Appetite 2015;95:571-576.
Wilbur 1981 \{published data only\}

Wilbur CS, Zifferblatt SM, Pinsky JL, Zifferblatt S. Healthy vending: a cooperative pilot research program to stimulate good health in the marketplace. Preventive Medicine 1981;10:85-93.

\section{Wilcox 2009 \{published data only\}}

Wilcox K, Vallen B, Block L, Fitzsimons GJ. Vicarious goal fulfillment: when the mere presence of a healthy option leads to an ironically indulgent decision. Journal of Consumer Research 2009;36:380-393.

\section{Wilson 2017 \{published data only\}}

Wilson NLW, Just DR, Swigert J, Wansink B. Food pantry selection solutions: a randomized controlled trial in clientchoice food pantries to nudge clients to targeted foods. Journal of Public Health 2017;39(2):366-372.

\section{Wolfenden 2015 \{published data only\}}

Wolfenden L, Kingsland M, Rowland BC, Dodds P, Gillham K, Sze LY, et al. Improving availability, promotion and purchase of fruit and vegetable and non sugar-sweetened drink products at community sporting clubs: a randomised trial. International Journal of Behavioral Nutrition and Physical Activity 2015;12:35.

\section{Wordell 2012 \{published data only\}}

Wordell D, Daratha K, Mandal B, Bindler R, Butkus SN. Changes in a middle school food environment affect food behavior and food choices. Journal of the Academy of Nutrition and Dietetics 2012;112:137-141.

\section{Zeinstra 2010 \{published data only\}}

Zeinstra GG, Renes RJ, Koelen MA, Kok FJ, de Graaf C. Offering choice and its effect on Dutch children's liking and consumption of vegetables: a randomized controlled trial. American Journal of Clinical Nutrition 2010;91:349-356.

\section{References to studies awaiting assessment}

Hua 2017 \{published data only\}

Hua SV, Kimmel L, VanEmmenes M, Taherian R, Remer G, Millman A, et al. Health promotion and healthier products increase vending purchases: a randomized factorial trial. Journal of the Academy of Nutrition and Dietetics 2017;117:1057-1065.

\section{Watanabe 2016 \{published data only\}}

Watanabe A, Fukuda Y. Effect of dish order on food intake in a buffet lunch among Japanese university students. JJHEP 2016;24(1):3-11.

\section{References to ongoing studies}

\section{Knowles 2017 \{published data only\}}

Knowles D, Brown K, Aldrovandi S. Investigating the proximity effect in a competitive food environment: a study protocol. osf.io/fgm4s/ (created 18 September 2017). 
Knowles 2018a \{published data only\}

Knowles D, Brown K, Aldrovandi S. Exploring the role of effort within the proximity effect: a study protocol. osf.io/eqt92/ (created 5 April 2018).

\section{Knowles 2018b \{published data only\}}

Knowles D, Brown K, Aldrovandi S. Exploring the role of visual salience within the proximity effect. osf.io/5gx9y/ (created 21 May 2018).

\section{Knowles 2018c \{published data only\}}

Knowles D, Brown K, Aldrovandi S. Does the proximity effect occur through non-conscious processes? A study protocol. osf.io/zn256/ (created 26 November 2018).

\section{Additional references}

\section{Allan 2017}

Allan J, Querstret D, Banas K, de Bruin M. Environmental interventions for altering eating behaviours of employees in the workplace: a systematic review. Obesity Reviews 2017;8(2):214-226.

\section{Anderson 2011}

Anderson LM, Petticrew M, Rehfuess E, Armstrong R, Ueffing E, Baker P, et al. Using logic models to capture complexity in systematic reviews. Research Synthesis Methods 2011;2:33-42.

\section{Anderson 2013}

Anderson LM, Oliver SR, Michie S, Rehfuess E, Noyes J, Shemilt I. Investigating complexity in systematic reviews of interventions by using a spectrum of methods. Journal of Clinical Epidemiology 2013;66:1223-9.

\section{Balshem 2011}

Balshem H, Helfand M, Schunemann HJ, Oxman AD, Kunze R, Brozek J, et al. GRADE guidelines: 3. Rating the quality of evidence. Journal of Clinical Epidemiology 2011;64:401-6.

\section{Bar-Hillel 2015}

Bar-Hillel M. Position effects in choice from simultaneous displays: a conundrum solved. Perspectives on Psychological Science 2015;10:419-33.

\section{Broers 2017}

Broers VJV, De Breucker C, Van den Broucke S, Luminet O. A systematic review and meta-analysis of the effectiveness of nudging to increase fruit and vegetable choice. European Journal of Public Health 2017;27:912-920.

\section{Bucher 2016}

Bucher T, Collins C, Rollo ME, McCaffrey TA, De Vlieger N, Van der Bend D, et al. Nudging consumers towards healthier choices: a systematic review of positional influences on food choice. British Journal of Nutrition 2016;115:2252-63.

\section{Cadario in press}

Cadario R, Chandon P. Which healthy eating nudges work best? A meta-analysis of field experiments. Marketing Science (in press).

\section{Cameron 2016}

Cameron AJ, Charlton E, Ngan WW, Sacks G. A systematic review of the effectiveness of supermarket-based interventions involving product, promotion, or place on the healthiness of consumer purchases. Current Nutrition Reports 2016;5(3):129-138.

\section{Chernev 2011}

Chernev A. Product assortment and consumer choice: an interdisciplinary review. Foundations and Trends in Marketing 2011;6:1-61.

\section{Cohen 2016}

Cohen DA, Lesser LI. Obesity prevention at the point of purchase. Obesity Reviews 2016;17:389-396.

\section{Crockett 2018}

Crockett RA, King SE, Marteau TM, Prevost AT, Stubbs B, Bignardi $G$, et al. Nutritional labelling for healthier food or non-alcoholic drink purchasing and consumption. Cochrane Database of Systematic Reviews 2018, Issue 2. [DOI: 10.1002/14651858.CD009315.pub2]

\section{Dalenberg 2014}

Dalenberg JR, Nanetti L, Renken RJ, de Wijk RA, ter Horst GJ. Dealing with consumer differences in liking during repeated exposure to food; typical dynamics in rating behavior. PLOS ONE 2014;9(3):e93350.

\section{Deeks 2011}

Deeks JJ, Higgins JPT, Altman DG. Chapter 9: Analysing data and undertaking meta-analyses. In: Higgins JP, Green S, editor(s). Cochrane Handbook for Systematic Reviews of Interventions Version 5.1.0 (updated March 2011). The Cochrane Collaboration, 2011. Available from handbook.cochrane.org.

\section{Department of Health 2011}

Department of Health. Statement of the calorie reduction expert group (policy paper). www.gov.uk/government/ publications/statement-of-the-calorie-reduction-expert-group.

\section{Department of Health and Social Care 2018}

HM Government. Childhood obesity: a plan for action. Chapter 2. Accessed from: https://assets.publishing.service.gov.uk/ government/uploads/system/uploads/attachment_data/ file/718903/childhood-obesity-a-plan-for-action-chapter-2.pdf. Department of Heath and Social Care, 2018.

\section{Egger 1997}

Egger M, Smith GD, Schneider M, Minde C. Bias in meta-analysis detected by a simple, graphical test. BMJ 1997;315:629-34.

\section{Ejlerskov 2018a}

Ejlerskov KT, Stead M, Adamson A, White M, Adams J. The nature of UK supermarkets' policies on checkout food and associations with healthfulness and type of food displayed: cross-sectional study. International Journal of Behavioral Nutrition and Physical Activity 2018;15:52. 


\section{Ejlerskov 2018b}

Ejlerskov KT, Sharp SJ, Stead M, Adamson AJ, White M, Adams J. Supermarket policies on less-healthy food at checkouts: natural experimental evaluation using interrupted time series analyses of purchases. PLOS Medicine 2018;15(12):e1002712.

\section{Eldridge 2016}

Eldridge S, Campbell M, Campbell M, Dahota A, Giraudeau B, Higgins JPT, et al. Revised Cochrane risk of bias tool for randomized trials (RoB 2.0): additional considerations for cluster-randomized trials. www.riskofbias.info (accessed 1 December 2016).

\section{GBD 2018}

GBD 2017 Risk Factors Collaborators. Global, regional, and national comparative risk assessment of 84 behavioural, environmental and occupational, and metabolic risks or clusters of risks for 195 countries and territories, 1990-2017: a systematic analysis for the Global Burden of Disease Study 2017. Lancet 2018;392:1923-94.

\section{GRADEpro GDT 2015 [Computer program]}

McMaster University, 2015 (developed by Evidence Prime, Inc.). Available from gradepro.org. GRADEpro GDT: GRADEpro Guideline Development Tool [Software]. McMaster University, 2015 (developed by Evidence Prime, Inc.). Available from gradepro.org, 2015.

\section{Grech 2015}

Grech A, Allman-Farinelli M. A systematic literature review of nutrition interventions in vending machines that encourage consumers to make healthier choices. Obesity Reviews 2015;16:1030-41.

\section{Guyatt 2011}

Guyatt G, Oxman AD, Akl EA, Kunz R, Vist G, Brozek J, et al. GRADE guidelines: 1 . Introduction - GRADE evidence profiles and summary of findings tables. Journal of Clinical Epidemiology 2011;64(4):383-394.

\section{Hall 2014}

Hall PA, Marteau TM. Executive function in the context of chronic disease prevention: theory, research and practice. Preventive Medicine 2014;68:44-50.

\section{Han 2014}

Han T, Alexander M, Niggebrugge A, Hollands GJ, Marteau TM. Impact of tobacco outlet density and proximity on smoking cessation: a longitudinal observational study in two English cities. Health \& Place 2014;27:45-50.

\section{Higgins 2011}

Higgins JPT, Green S (editors). Cochrane Handbook for Systematic Reviews of Interventions Version 5.1.0 (updated March 2011). The Cochrane Collaboration, 2011. Available from handbook.cochrane.org. The Cochrane Collaboration.

\section{Higgins 2016a}

Higgins JPT, Savović J, Page MJ, Sterne JAC. Revised Cochrane risk of bias tool for randomized trials (RoB 2.0). www.riskofbias.info (accessed 1 December 2016).

\section{Higgins 2016b}

Higgins JPT, Altman D, Curtin F, Li T, Senn S. Revised Cochrane risk of bias tool for randomized trials (RoB 2.0): additional considerations for cross-over trials. www.riskofbias.info (accessed 1 December 2016).

\section{Hollands 2013a}

Hollands GJ, Shemilt I, Marteau TM, Jebb SA, Kelly MP, Nakamura R, et al. Altering micro-environments to change population health behaviour: towards an evidence base for choice architecture interventions. BMC Public Health 2013;13:1218.

\section{Hollands 2013b}

Hollands GJ, Shemilt I, Marteau TM, Jebb SA, Kelly MP, Nakamura R, et al. Altering choice architecture to change population health behaviour: a large-scale conceptual and empirical scoping review of interventions within microenvironments. Cambridge (UK): University of Cambridge, 2013

\section{Hollands 2015}

Hollands GJ, Shemilt I, Marteau TM, Jebb SA, Lewis HB, Wei Y, et al. Portion, package or tableware size for changing selection and consumption of food, alcohol and tobacco. Cochrane Database of Systematic Reviews 2015, Issue 9. [DOI: 10.1002/14651858.CD011045.pub2]

\section{Hollands 2016}

Hollands GJ, Marteau TM, Fletcher PC. Nonconscious processes in changing health-related behaviour: a conceptual analysis and framework. Health Psychology Review 2016;10(4):381-94. [DOI: 10.1080/17437199.2015.1138093]

\section{Hollands 2017a}

Hollands GJ, Bignardi G, Johnston M, Kelly MP, Ogilvie D, Petticrew M, et al. The TIPPME intervention typology for changing environments to change behaviour. Nature Human Behaviour 2017;1:0140.

\section{Hollands 2017b}

Hollands GJ, Carter P, Shemilt I, Marteau TM, Jebb SA, Higgins J, et al. Altering the availability or proximity of food, alcohol and tobacco products to change their selection and consumption. Cochrane Database of Systematic Reviews 2017, Issue 3. [DOI: 10.1002/14651858.CD012573]

\section{Hollands 2018}

Hollands GJ, Cartwright E, Pilling M, Pechey R, Vasiljevic M, Jebb SA, et al. Impact of reducing portion sizes in worksite cafeterias: a stepped wedge randomised controlled pilot trial. International Journal of Behavioral Nutrition and Physical Activity 2018;15:78.

\section{Hunter 2018}

Hunter JA, Hollands GJ, Couturier DL, Marteau TM. Effect of snack-food proximity on intake in general population samples with higher and lower cognitive resource. Appetite 2018;121:337-347. 


\section{Jones 2016}

Jones K, Hollands GJ, Shemilt I, Doyle J, Armstrong R. Planning and implementing a targeted and strategic dissemination plan for a Cochrane review: a case study. Journal of Public Health 2016;38(3):630-2. [DOI: 10.1093/pubmed/fdw045]

\section{Kroese 2016}

Kroese FM, Marchiori DR, de Ridder DT. Nudging healthy food choices: a field experiment at the train station. Journal of Public Health 2016;38(2):e133-7.

\section{Lorenc 2013}

Lorenc T, Petticrew M, Welch V, Tugwell P. What types of interventions generate inequalities? Evidence from systematic reviews. Journal of Epidemiology and Community Health 2013;67(2):190-3.

\section{Maas 2012}

Maas J, de Ridder DTD, de Vet E, de Wit JBF. Do distant foods decrease intake? The effect of food accessibility on consumption. Psychology \& Health 2012;27 (Suppl 2):59-73.

\section{Marteau 2012}

Marteau TM, Hollands GJ, Fletcher PC. Changing human behaviour to prevent disease: the importance of targeting automatic processes. Science 2012;337(6101):1492-5.

\section{Marteau 2015}

Marteau TM, Hollands GJ, Kelly MP. Changing population behavior and reducing health disparities: exploring the potential of "choice architecture" interventions. In: Kaplan RM, Spittel M, David DH editor(s). Emerging Behavioral and Social Science Perspectives on Population Health. Bethesda: National Institutes of Health/Agency for Healthcare Research and Quality, 2015:105-26.

\section{McGill 2015}

McGill R, Anwar E, Orton L, Bromley H, Lloyd-Williams F, O'Flaherty M, et al. Are interventions to promote healthy eating equally effective for all? Systematic review of socioeconomic inequalities in impact. BMC Public Health 2015;15(1):457.

\section{Miwa 2014}

Miwa M, Thomas J, O'Mara-Eves A, Ananiadou S. Reducing systematic review workload through certainty-based screening. Journal of Biomedical Informatics 2014;51:242-53.

\section{Moher 2009}

Moher D, Liberati A, Tetzlaff J, Altman DG, The PRISMA Group. Preferred reporting items for systematic reviews and meta-analyses: The PRISMA statement. PLOS Medicine 2009;6(7):e1000097.

\section{Munafò 2018}

Munafò MR, Hollands GJ, Marteau TM. Open science prevents mindless science: lessons from a case of academic misconduct. BMJ 2018;363:k4309.

\section{Neal 2006}

Neal DT, Wood W, Quinn JM. Habits - a repeat performance. Current Directions in Psychological Science 2006;15:198-202.

\section{NHS 2018}

NHS/Change4life. 100 calorie snacks. www.nhs.uk/change4life/ food-facts/healthier-snacks-for-kids/100-calorie-snacks (accessed prior to 17 July 2019).

\section{O'Mara-Eves 2015}

O'Mara-Eves A, Thomas J, McNaught J, Miwa M, Ananiadou S. Using text mining for study identification in systematic reviews: a systematic review of current approaches. Systematic Reviews 2015;4:5.

\section{Pechey under review}

Pechey P, Hollands GJ, Carter P, Marteau TM. Altering the availability and position of products within physical microenvironments: a conceptual review and framework. Under review; preprint posted at doi.org/10.31234/osf.io/qzb3w.

\section{Pedregosa 2011}

Pedregosa F, Varoquaux G, Gramfort A, Michel V, Thirion B, Grisel O, et al. Scikit-learn: machine Learning in Python. Journal of Machine Learning Research 2011;12:2825-30.

\section{Petrescu 2016}

Petrescu DC, Hollands GJ, Ng YL, Couturier DL, Marteau TM. Public acceptability in the UK and USA of nudging to reduce obesity: the example of reducing sugar-sweetened beverages consumption. PLOS ONE 2016;11(6):e0155995.

\section{Public Health England 2018a}

Roberts C, Steer T, Maplethorpe N, Cox L, Meadows S, Nicholson S, et al. National Diet and Nutrition Survey results from years 7 and 8 (combined) of the rolling programme (2014/2015 to 2015/2016). www.gov.uk/government/statistics/ ndns-results-from-years-7-and-8-combined (accessed prior to 17 July 2019).

\section{Public Health England 2018b}

Chadborn T. Hospital vending machines: helping people make healthier choices. publichealthmatters.blog.gov.uk/2018/07/20/ hospital-vending-machines-helping-people-make-healthierchoices/.

\section{Reisch 2016}

Reisch LA, Sunstein CR. Do Europeans like nudges?. Judgment and Decision Making 2016;11:310-325.

\section{Reynolds 2019}

Reynolds JP, Archer S, Pilling M, Kenny M, Hollands GJ, Marteau TM. Public acceptability of nudging and taxing to reduce consumption of food, alcohol and tobacco: a population-based survey experiment. Social Science \& Medicine 2019;236:112395.

\section{Schünemann 2011}

Schünemann HJ, Oxman AD, Vist GE, Higgins JPT, Deeks JJ, Glasziou P, et al. Chapter 12: Interpreting results and drawing conclusions. In: Higgins JP, Green S, editor(s). Cochrane Handbook for Systematic Reviews of Interventions Version 5.1.0 (updated March 2011). The Cochrane Collaboration, 2011. Available from handbook.cochrane.org. 


\section{Scottish Government 2015}

Scottish Government. Criteria for the Healthcare Retail Standard. Accessed from: https://www.gov.scot/publications/ criteria-healthcare-retail-standard/ 2015.

\section{Sela 2009}

Sela A, Berger J, Liu W. Variety, vice, and virtue: how assortment size influences option choice. Journal of Consumer Research 2009;35(6):941-51.

\section{Shemilt 2013}

Shemilt I, Hollands GJ, Marteau TM, Jebb SA, Kelly MP, Nakamura R, et al. Economic instruments for population diet and physical activity behaviour change: a systematic scoping review. PLOS ONE 2013;8(9):e75070.

\section{Shemilt 2014}

Shemilt I, Simon A, Hollands GJ, Marteau TM, Ogilvie D, O'MaraEves $A$, et al. Pinpointing needles in giant haystacks: use of text mining to reduce impractical screening workload in extremely large scoping reviews. Research Synthesis Methods 2014;5(1):31-49.

\section{Sherk 2018}

Sherk A, Stockwell T, Chikritzhs T, Andréasson S, Angus C, Gripenberg J, et al. Alcohol consumption and the physical availability of take-away alcohol: systematic reviews and metaanalyses of the days and hours of sale and outlet density. Journal of Studies on Alcohol and Drugs 2018;79(1):58-67.

\section{Stok 2017}

Stok FM, Hoffmann S, Volkert D, Boeing H, Ensenauer R, Stelmach-Mardas M, et al. The DONE framework: Creation, evaluation, and updating of an interdisciplinary, dynamic framework 2.0 of determinants of nutrition and eating. PLOS ONE 2017;12:e0171077.

\section{Stringhini 2010}

Stringhini S, Sabia S, Shipley M, Brunner E, Nabi H, Kivimaki M, et al. Association of socioeconomic position with health behaviors and mortality. JAMA 2010;303(12):1159-66.

\section{Swinburn 1999}

Swinburn B, Egger G, Raza F. Dissecting obesogenic environments: the development and application of a framework for identifying and prioritizing environmental interventions for obesity. Preventive Medicine 1999;29(6):56370.

\section{Szaszi 2018}

Szaszi B, Palinkas A, Palfi B, Szollosi A, Aczel B. A systematic scoping review of the choice architecture movement: toward

\section{CHARACTERISTICS OF STUDIES}

Characteristics of included studies [ordered by study ID] understanding when and why nudges work. Journal of Behavioral Decision Making 2018;31(3):355-366.

\section{Thaler 2008}

Thaler RH, Sunstein CR. Nudge: Improving decisions about health, wealth, and happiness. New Haven: Yale University Press, 2008.

\section{Thomas 2010 [Computer program]}

Thomas J, Brunton J, Graziosi S. EPPI-Reviewer 4.0: software for research synthesis. EPPI-Centre Software. London: Social Science Research Unit, Institute of Education, 2010.

\section{Trope 2010}

Trope Y, Liberman N. Construal-level theory of psychological distance. Psychological Review 2010;117:440-63.

\section{United Nations 1989}

United Nations. Convention on the rights of the child. treaties.un.org/doc/Publication/UNTS/Volume\%201577/ v1577.pdf.

\section{Vermeer 2009}

Vermeer WM, Steenhuis IHM, Seidell JC. From the point-ofpurchase perspective: a qualitative study of the feasibility of interventions aimed at portion-size. Health Policy 2009;90:73-80.

\section{Viechtbauer 2010}

Viechtbauer W. Conducting meta-analyses in R with the metafor package. Journal of Statistical Software 2010;36(3):1-48.

\section{Welch 2012}

Welch V, Petticrew M, Tugwell P, Moher D, O'Neill J, Waters E, et al. PRISMA-Equity Bellagio Group. PRISMA-Equity 2012 extension: reporting guidelines for systematic reviews with a focus on health equity. PLOS Medicine 2012;9(10):e1001333.

\section{WHO 2016}

World Health Organization. World Health Statistics 2016. Geneva: World Health Organization, 2016.

\section{References to other published versions of this review Hollands 2017}

Hollands GJ, Carter P, Shemilt I, Marteau TM, Jebb SA, Higgins J, et al. Altering the availability or proximity of food, alcohol and tobacco products to change their selection and consumption. Cochrane Database of Systematic Reviews 2017, Issue 3. [DOI: 10.1002/14651858.CD012573]

* Indicates the major publication for the study 
Cohen 2015

Methods

Participants
Study design: Between-participants cluster-randomised controlled trial

Setting: Field setting

Setting type: School cafeteria

Geographical region: USA

Number of enrolled participants: 1587 (actively consented in groups of interest) (within 10 study sites)

Number (\%) of enrolled participants completing the study and included in analysis: 1587 (100\%)

Study completers - mean age (SD): 11.8 (not reported)

Study completers - sex: $55.4 \%$ female

Specific social or cultural characteristics: Low-income schoolchildren of elementary and middle school age

Socioeconomic status context: High deprivation

Inclusion criteria: None reported

Exclusion criteria: None reported

\section{Interventions}

Type of proximity intervention: Proximity from start of queue line (order encountered along line (e.g. queue or aisle))

\section{Manipulated product type: Food}

Characteristics of manipulated products: Vegetables

Duration of exposure to intervention: $>1$ day

Study arms: Smart cafe intervention which included vegetables being offered at the start of the lunch line (along with: signage and images promoting fruits and vegetables prominently displayed, fruits placed in attractive containers, other fruit options placed next to the cash registers, white milk placed prominently in front of sugar-sweetened milk); control condition with vegetables being offered later in the lunch line

\section{Number of comparisons analysed: 1}

Comparisons analysed: Vegetables offered at the start of the lunch line versus vegetables offered later in the lunch line

\section{Concurrent intervention components in factorial design: No}

Concurrent intervention components confounded with comparison of interest: Yes (signage and images promoting fruits and vegetables prominently displayed, fruits placed in attractive containers, other fruit options placed next to the cash registers, white milk placed prominently in front of sugar-sweetened milk)

Outcomes reported in study: Proportion of total participants selecting vegetables; proportion of total participants selecting an entrée; proportion of total participants selecting fruit; mean cups of vegetables consumed; mean cups of fruit consumed; proportion of entrée consumed; proportion of fruit consumed; proportion of vegetables consumed

Selection outcome analysed: Proportion of total participants selecting vegetables

Measurement of selection outcome: Objective 
Timing of selection outcome measurement: Longer term (> 1 day)

Consumption outcome analysed: Mean cups of vegetables consumed (clear manipulation of proximity of vegetables)

Measurement of consumption outcome: Objective

Timing of consumption outcome measurement: Longer term ( $>1$ day)

Secondary outcome: Entrée selection; entrée consumption

Funding source

Funded by grants from Arbella Insurance and the Nutritional Epidemiology of Cancer Education and Career Development Program. No conflicts of interest declared and it was stated that funders had no role in the study.

Notes Outcome data reported relate to only participants providing active consent. Data from participants within 2 additional study arms that had previously been randomised to a school chef intervention (ineligible, containing $4 / 14$ schools within the larger study) 4 months prior to the randomisation assignment pertinent to the current analysis were not included.

Engell 1996 (S1)

Methods Study design: Between-participants randomised controlled trial

Participants Setting: Laboratory setting

Setting type: Naturalistic laboratory (dining room in research centre)

Geographical region: USA

Number of enrolled participants: 36

Number (\%) of enrolled participants completing the study and included in analysis: $36(100 \%)$

Study completers - mean age (SD): Not provided

Study completers - sex: $0 \%$ female

Specific social or cultural characteristics: Employees of US Army Natick Research Center

Socioeconomic status context: Low deprivation

Inclusion criteria: Male; employees of US Army Natick Research Center

Exclusion criteria: None reported

Interventions Intervention type: Proximity

Type of proximity intervention: Proximity from positioned participant (table at which seated) (distance from set point)

\section{Manipulated product type: Food}

Characteristics of manipulated products: Water

Duration of exposure to intervention: $\leq 1$ day

Study arms: Water available within reach in pitcher on table; water available within immovable dispenser $610 \mathrm{~cm}$ (20 feet) from the table; water available within immovable dispenser $1220 \mathrm{~cm}$ (40 feet) from the table

\section{Number of comparisons analysed: 2}


Engell 1996 (S1) (Continued)

\section{Comparisons analysed:}

Comparison 1: Water on table versus water $610 \mathrm{~cm}$ (20 feet) from table

Comparison 2: Water on table versus water $1220 \mathrm{~cm}$ (40 feet) from table

Concurrent intervention components in factorial design: No

Concurrent intervention components confounded with comparison of interest: No

\begin{tabular}{ll}
\hline Outcomes & Outcomes reported in study: Water intake $(\mathrm{g})$ \\
& Selection outcome analysed: N/A \\
Measurement of selection outcome: N/A & Timing of selection outcome measurement: N/A \\
Consumption outcome analysed: Water intake $(\mathrm{g})$ \\
Measurement of consumption outcome: Objective \\
Timing of consumption outcome measurement: Immediate ( $\leq 1$ day) \\
Secondary outcome: N/A \\
\hline Funding source \\
\hline Fotes
\end{tabular}

Engell 1996 (S2)

Methods Study design: Between-participants randomised controlled trial

Participants

Setting: Laboratory setting

Setting type: Naturalistic laboratory (dining room in research centre)

Geographical region: USA

Number of enrolled participants: 60

Number (\%) of enrolled participants completing the study and included in analysis: $60(100 \%)$

Study completers - mean age (SD): Not provided

Study completers - sex: $0 \%$ female

Specific social or cultural characteristics: Employees of US Army Natick Research Center

Socioeconomic status context: Low deprivation

Inclusion criteria: Male; employees of US Army Natick Research Center

Exclusion criteria: None reported

Interventions Intervention type: Proximity

Type of proximity intervention: Proximity from positioned participant (table at which seated) (distance from set point)

Manipulated product type: Food 
Characteristics of manipulated products: Water

Duration of exposure to intervention: $\leq 1$ day

Study arms: Water available within reach on table; water available $610 \mathrm{~cm}$ (20 feet) from table. (In $2 \times 3$ factorial design with social manipulation: no confederate; confederate drinking small amount; confederate drinking large amount)

Number of comparisons analysed: 1

Comparisons analysed: Water on table versus water $610 \mathrm{~cm}$ (20 feet) from table

Concurrent intervention components in factorial design: Yes (social manipulation: no confederate; confederate drinking small amount; confederate drinking large amount)

Concurrent intervention components confounded with comparison of interest: No

Outcomes

Outcomes reported in study: Water intake $(\mathrm{g})$

Selection outcome analysed: N/A

Measurement of selection outcome: N/A

Timing of selection outcome measurement: N/A

Consumption outcome analysed: Water intake (g)

Measurement of consumption outcome: Objective

Timing of consumption outcome measurement: Immediate ( $\leq 1$ day)

Secondary outcome: N/A

Notes

Fiske 2004

Methods Study design: Between-participants cluster-randomised controlled trial

Participants

Setting: Field setting

Setting type: Teachers lounges in schools

Geographical region: USA

Number of enrolled participants: N/A (10 vending machines)

Number (\%) of enrolled participants completing the study and included in analysis: N/A (100\% of vending machines)

Study completers - mean age (SD): N/A

Study completers - sex: N/A

Specific social or cultural characteristics: School teachers lounges

Socioeconomic status context: Low deprivation

Inclusion criteria: Ability of vending machines to electronically track sales on site 
Fiske 2004 (Continued)

Exclusion criteria: None reported

Interventions

Intervention type: Availability

Type of availability intervention: Relative number (proportion) of less-healthy (to healthier) options: higher and lower availability of high-fat (relative to low-fat) items in vending machines

Manipulated product type: Food

Characteristics of manipulated products: High-fat snack food (containing over $5 \mathrm{~g}$ of fat) or gum products, being replaced by low-fat products

\section{Duration of exposure to intervention: $>1$ day}

Study arms: No changes made, vending machines with 33 snack food and gum items including 5 lowfat items ( $<5 \mathrm{~g}$ of fat); reductions in available high-fat items due to including 3 additional low-fat items, plus labels highlighting prices of low-fat items; reductions in available high-fat items due to including 3 additional low-fat items, plus labels, plus large motivational signs

\section{Number of comparisons analysed: 1}

Comparisons analysed: Higher availability of high-fat items, vending machines with 33 snack food and gum items including 5 low-fat items ( $<5 \mathrm{~g}$ of fat) versus lower availability of high-fat items, vending machines with 33 snack food and gum items including 8 low-fat items ( $<5 \mathrm{~g}$ of fat) (combining 2 study conditions)

\section{Concurrent intervention components in factorial design: No}

Concurrent intervention components confounded with comparison of interest: Yes (labels (1 condition) and labels plus signs (1 condition))

Outcomes

Outcomes reported in study: Total items sold; number of a range of low-fat items sold; number of high-fat items sold; total dollar sales for low-fat items

Selection outcome analysed: Mean number of high-fat ('Other') items sold in intervention weeks

Measurement of selection outcome: Objective

Timing of selection outcome measurement: Longer term ( $>1$ day)

Consumption outcome analysed: No

Measurement of consumption outcome: N/A

Timing of consumption outcome measurement: N/A

Secondary outcome: N/A

Funding source

United States Department of Agriculture/Agricultural Research Service (USDA/ARS) and National Cancer Institute. The authors did not report on conflicts of interest.

Notes

The vending machines were in different schools, but there was no mention of school size, number of teachers who purchased products, etc., and data were reported at site-level. Outcome data (standard deviations) requested from the authors (11/2017) was not received.

\begin{tabular}{ll}
\hline Methods & Study design: Between-participants cluster-randomised controlled trial \\
\hline Participants & Setting: Field setting \\
\end{tabular}


Setting type: Supermarkets

Geographical region: USA

Number of enrolled participants: N/A (8 supermarkets)

Number (\%) of enrolled participants completing the study and included in analysis: N/A (100\% of supermarkets)

Study completers - mean age (SD): N/A

Study completers - sex: N/A

Specific social or cultural characteristics: High-minority, low-income shoppers

Socioeconomic status context: High deprivation

Inclusion criteria: Eligible supermarkets had to be located in a low-to-moderate income census tract, located in an area of below-average supermarket density, or located in an area having a supermarket customer base with $>50 \%$ living in a low-income census tract

Exclusion criteria: None reported

Interventions Intervention type: Availability

Type of availability intervention: Relative number (proportion) of less-healthy (to healthier) options: higher and lower availability (in checkout refrigerators) of full-calorie beverages (vs reduced-calories beverages)

\section{Manipulated product type: Food}

Characteristics of manipulated products: Full-calorie beverages in checkout refrigerators. In context of wide range of other products in shops (including other manipulated categories such as milk and frozen meals)

\section{Duration of exposure to intervention: $>1$ day}

Study arms: Targeted products were 1) milk, 2) ready-to-eat cereal, 3) beverages (split into in-aisle beverages and checkout cooler beverages for the intervention), and 4) single-serving frozen meals. The intervention consisted of 4 major marketing strategies used across all categories, with placement as the dominant strategy and promotion as the secondary strategy. Strategies included 1) multiple facings: increased the number of facings of the recommended products; 2) prime placement: placed recommended products at eye/arm level and in the middle of the category aisle and reordered types of milk so that $2 \%$ milk was located on the left-hand side of the dairy case followed by $1 \%$, skim, and then whole milk; 3) signage: placed call-out signs with the recommended product's name and price, and shelf runners below recommended products; and 4) secondary placement: mimicked shelf strategies ( 1 and 2 ) in all secondary placements (end caps, dead space stacks, etc.). In addition, other strategies were used as appropriate to the category, including 5) cross promotion (cereal and beverages only): displayed recommended products in 2 product categories together, through dead space stacks and end caps (e.g. cereal and bananas, soda and water); and 6) taste-testing (milk only): offered free samples of recommended products to increase shoppers' exposure to healthier options. Control supermarkets received no intervention.

\section{Number of comparisons analysed: 1}

Comparisons analysed: More facings (shelf space) for full-calorie beverages (vs reduced-calorie beverages) in checkout refrigerators versus fewer facings (less shelf space) for full-calorie beverages (vs reduced-calorie beverages) in checkout refrigerators

\section{Concurrent intervention components in factorial design: No}

Concurrent intervention components confounded with comparison of interest: Yes (prime placement of zero-calorie options, placing water on the top shelf of refrigerator and diet beverages on middle 2 shelves) 
Foster 2014 (Continued)

Outcomes

Outcomes reported in study: Weekly sales data for each product category (milk; cereal; frozen meals; in-aisle beverages; checkout cooler beverages)

Selection outcome analysed: Weekly sales data for full-calorie/regular checkout cooler beverages

Measurement of selection outcome: Objective

Timing of selection outcome measurement: Longer term (> 1 day)

Consumption outcome analysed: No

Measurement of consumption outcome: N/A

Timing of consumption outcome measurement: N/A

Secondary outcome: N/A

Funding source Supported by grants from the Robert Wood Johnson Foundation and the United States Department of Agriculture. The authors reported that there were no conflicts of interest.

Notes

Checkout cooler beverages were selected as outcome of interest as targeted by the least confounded availability intervention with the fewest concurrent intervention components. Full-calorie beverages (vs low-calorie and water) within that wider category selected as primary outcome due to relative importance of reducing consumption of high-energy products versus increasing consumption of low-energy products.

\begin{tabular}{ll}
\hline Methods & Study design: Between-participants cluster-randomised controlled trial \\
\hline Participants & Setting: Field setting \\
Setting type: School cafeteria & Geographical region: USA \\
& Number of enrolled participants: 10 study sites (randomised into a fruit intervention ( $\mathrm{n}=4$ ), veg- \\
& etable intervention ( $=3$ ), or control group $(\mathrm{n}=3)$ ) \\
& Number (\%) of enrolled participants completing the study and included in analysis: 7 sites with \\
& 2108 children in grades 5 to 8 (70\%) (this paper only presented results for 2 out of 3 conditions: fruit in- \\
& tervention ( $\mathrm{n}=4$ ) versus control ( $=3)$ ) \\
& Study completers - mean age (SD): Not reported \\
& Study completers - sex: Not reported \\
& Specific social or cultural characteristics: Students from urban and rural middle schools \\
& Socioeconomic status context: Both high and low deprivation \\
Inclusion criteria: Middle schools (grades 5 to 8 ) from upstate New York \\
Exclusion criteria: None reported
\end{tabular}

Interventions

Intervention type: Proximity

Type of proximity intervention: Proximity from start of line

Manipulated product type: Food 


\section{Characteristics of manipulated products: Fruit}

Duration of exposure to intervention: $\geq 1$ day

Study arms: Fruit is placed first in the line of foods offered; control with no changes (presumed to be later in line)

\section{Number of comparisons analysed: 1}

Comparisons analysed: Fruit is placed first in the line of foods offered versus control with no changes (the schools were offered Smarter Lunchrooms training postintervention)

\section{Concurrent intervention components in factorial design: No}

Concurrent intervention components confounded with comparison of interest: Yes (further changes to enhance convenience, visibility, and attractiveness were: at least 2 varieties of fruit were offered; fruit was offered in at least 2 separate locations; cut fruits were displayed in small, attractive cups; whole fruits were displayed in a large, attractive fruit bowl at eye level; fruits were labelled with creative names; creative fruit names were displayed on monthly and daily menus; "fruit factoids" were displayed on dry-erase boards at eye level)

Outcomes

Outcomes reported in study: Fruit, vegetable, and milk selection and consumption based on plate waste data; mean number of items selected and consumed; proportion of lunch trays that contained any type of fruit

Selection outcome analysed: Mean number of fruit items selected

Measurement of selection outcome: Objective

Timing of selection outcome measurement: Immediate ( $\leq 1$ day)

Consumption outcome analysed: Mean number of fruit items consumed

Measurement of consumption outcome: Objective

Timing of consumption outcome measurement: Immediate ( $\leq 1$ day)

Secondary outcome: Vegetable and milk selection and consumption

Funding source

Supported by Agriculture and Food Research Initiative Grant no. 2012-68001-19604 from the US Department of Agriculture National Institute of Food and Agriculture, Childhood Obesity Prevention: Integrated Research, Education, and Extension to Prevent Childhood Obesity-A2101

Notes

Although this study also randomised 3 of the 10 schools to a vegetable intervention, outcome data for these 3 schools were not reported in this publication - only fruit intervention results were reported. Publication of the remaining data will be monitored in future updates. Secondary outcomes of vegetable and milk selection and consumption reported in this paper were secondary observations in relation to the intervention focused on promoting fruit. Reports that ensuring availability of at least 2 choices of fruit was already being met in most schools, so while proximity was manipulated, availability was seemingly not altered.

Hunter 2018 (S1)

Methods Study design: Between-participants randomised controlled trial

Participants

Setting: Laboratory setting

Setting type: Laboratory (multipurpose room in community setting)

Geographical region: UK 
Hunter 2018 (S1) (Continued)

Number of enrolled participants: 159

Number (\%) of enrolled participants completing the study and included in analysis: $100 \%$

Study completers - mean age (SD): 38.4 (15.2)

Study completers - sex: $63.5 \%$ female

Specific social or cultural characteristics: General public sample

Socioeconomic status context: Both high and low deprivation

Inclusion criteria: 18 years or over

Exclusion criteria: Food allergies or intolerance

Interventions Intervention type: Proximity

Type of proximity intervention: Proximity from positioned participant (chair at which seated) (distance from set point)

Manipulated product type: Food

Characteristics of manipulated products: Chocolate M\&Ms

Duration of exposure to intervention: $\leq 1$ day

Study arms: $1000 \mathrm{~g}$ of chocolate M\&Ms in a transparent 1-litre bowl placed $20 \mathrm{~cm}$ from seated participants' right armrest; $1000 \mathrm{~g}$ of chocolate M\&Ms in a transparent 1-litre bowl placed $70 \mathrm{~cm}$ from seated participants' right armrest

Number of comparisons analysed: 1

Comparisons analysed: M\&Ms placed at $20 \mathrm{~cm}$ from participant versus M\&Ms placed at $70 \mathrm{~cm}$ from participant

Concurrent intervention components in factorial design: No

Concurrent intervention components confounded with comparison of interest: No

Outcomes

Outcomes reported in study: Proportion of total participants consuming any M\&Ms (specified as primary outcome); mean amount of snacks consumed (grams)

Selection outcome analysed: N/A

Measurement of selection outcome: N/A

Timing of selection outcome measurement: N/A

Consumption outcome analysed: Proportion of total participants consuming any M\&Ms

Measurement of consumption outcome: Objective

Timing of consumption outcome measurement: Immediate ( $\leq 1$ day)

Secondary outcome: N/A there were no conflicts of interest. 
Hunter 2018 (S2)

\begin{tabular}{ll}
\hline Methods & Study design: Between-participants randomised controlled trial \\
\hline Participants & Setting: Laboratory setting \\
Setting type: Laboratory (multipurpose room in community setting) \\
Geographical region: UK \\
Number of enrolled participants: 246 \\
Number (\%) of enrolled participants completing the study and included in analysis: $100 \%$ \\
Study completers - mean age (SD): 36.2 (13) \\
Study completers - sex: $56.5 \%$ female \\
Specific social or cultural characteristics: General public sample \\
Socioeconomic status context: Both high and low deprivation \\
Inclusion criteria: 18 years or over \\
Exclusion criteria: Food allergies or intolerance
\end{tabular}

Type of proximity intervention: Proximity from positioned participant (chair at which seated) (distance from set point)

Manipulated product type: Food

Characteristics of manipulated products: Chocolate M\&Ms

Duration of exposure to intervention: $\leq 1$ day

Study arms: $1000 \mathrm{~g}$ of chocolate M\&Ms in a transparent 1-litre bowl placed $20 \mathrm{~cm}$ from seated participants' right armrest; $1000 \mathrm{~g}$ of chocolate M\&Ms in a transparent 1-litre bowl placed $70 \mathrm{~cm}$ from seated participants' right armrest. (In factorial $2 \times 2$ design with manipulation of cognitive load: cognitive load earlier in session; cognitive load later in session)

\title{
Number of comparisons analysed: 1
}

Comparisons analysed: M\&Ms placed at $20 \mathrm{~cm}$ from participant versus M\&Ms placed at $70 \mathrm{~cm}$ from participant

Concurrent intervention components in factorial design: Yes (cognitive load earlier in session; cognitive load later in session)

Concurrent intervention components confounded with comparison of interest: No mary outcome); mean amount of snacks consumed (grams)

\author{
Selection outcome analysed: N/A \\ Measurement of selection outcome: N/A \\ Timing of selection outcome measurement: N/A \\ Consumption outcome analysed: Proportion of total participants consuming any M\&Ms \\ Measurement of consumption outcome: Objective \\ Timing of consumption outcome measurement: Immediate ( $\leq 1$ day)
}


Hunter 2018 (S2) (Continued)

\section{Secondary outcome: N/A}

Funding source

UK Medical Research Council and Raymond and Beverly Sackler Foundation. The authors reported that there were no conflicts of interest.

Notes

Hunter 2019

Methods Study design: Between-participants randomised controlled trial

Participants

Setting: Laboratory setting

Setting type: Laboratory (multipurpose room in community setting)

Geographical region: UK

Number of enrolled participants: 249

Number (\%) of enrolled participants completing the study and included in analysis: $99.6 \%$

Study completers - mean age (SD): 35.7 (12.4)

Study completers - sex: $49.4 \%$ female

Specific social or cultural characteristics: General public sample

Socioeconomic status context: Both high and low deprivation

Inclusion criteria: 18 years or over

Exclusion criteria: Food allergies or intolerance

Interventions Intervention type: Proximity

Type of proximity intervention: Proximity from positioned participant (chair at which seated) (distance from set point)

\section{Manipulated product type: Food}

Characteristics of manipulated products: Chocolate M\&Ms

Duration of exposure to intervention: $\leq 1$ day

Study arms: $1000 \mathrm{~g}$ of chocolate M\&Ms in a transparent 1-litre bowl placed $20 \mathrm{~cm}$ from seated participants' right armrest; $1000 \mathrm{~g}$ of chocolate M\&Ms in a transparent 1-litre bowl placed $70 \mathrm{~cm}$ from seated participants' right armrest. (In factorial $2 \times 2$ design with manipulation of proximity of bowl of raisins: bowl of raisins placed at $20 \mathrm{~cm}$; bowl of raisins placed at $70 \mathrm{~cm}$ )

Number of comparisons analysed: 1

Comparisons analysed: M\&Ms placed at $20 \mathrm{~cm}$ from participant versus M\&Ms placed at $70 \mathrm{~cm}$ from participant

Concurrent intervention components in factorial design: Yes (bowl of raisins placed at $20 \mathrm{~cm}$; bowl of raisins placed at $70 \mathrm{~cm}$ )

Concurrent intervention components confounded with comparison of interest: No

Outcomes

Outcomes reported in study: Proportion of total participants consuming any M\&Ms (specified as primary outcome); mean amount of M\&Ms consumed (grams); proportion of total participants consuming any raisins; mean amount of M\&Ms consumed (grams) 
Hunter 2019 (Continued)

\section{Selection outcome analysed: N/A}

Measurement of selection outcome: N/A

Timing of selection outcome measurement: N/A

Consumption outcome analysed: Proportion of total participants consuming any M\&Ms

Measurement of consumption outcome: Objective

Timing of consumption outcome measurement: Immediate ( $\leq 1$ day)

Secondary outcome: N/A there were no conflicts of interest.

Notes

\section{Kocken 2012}

\begin{tabular}{ll}
\hline Methods & Study design: Between-participants cluster-randomised controlled trial \\
\hline Participants & Setting: Field setting \\
Setting type: School setting, vending machines & Geographical region: The Netherlands \\
& Number of enrolled participants: N/A (40 schools) \\
& Number (\%) of enrolled participants completing the study and included in analysis: N/A (interven- \\
tion = 13 schools, control = 15 schools) (70\%) \\
Study completers - mean age (SD): N/A \\
Study completers - sex: N/A \\
Specific social or cultural characteristics: Schoolchildren \\
Socioeconomic status context: High deprivation \\
Inclusion criteria: None reported \\
Exclusion criteria: None reported
\end{tabular}

Interventions Intervention type: Availability

Type of availability intervention: Relative number (proportion) of less-healthy (to healthier) options: higher and lower availability of unfavourable extra foods (> $170 \mathrm{kcal}$ ) (relative to lower-calorie options) in vending machines

\section{Manipulated product type: Food}

Characteristics of manipulated products: Unfavourable extra foods (i.e. products with empty calories that deliver only energy and no important vitamins or minerals) ( $>170 \mathrm{kcal})$, replaced by $<100$ $\mathrm{kcal}$ (favourable) and moderately unfavourable (100 to $170 \mathrm{kcal}$ ) foods. In context of also replacing unfavourable beverages with favourable beverages.

Duration of exposure to intervention: $>1$ day 
Study arms: Unfavourable, high-energy foods (> $170 \mathrm{kcal}$ ) in vending machines were replaced by < $100 \mathrm{kcal}$ (favourable) and moderately unfavourable (100 to $170 \mathrm{kcal}$ ) foods, with categorisation based on Netherlands Nutrition Centre recommendations. Intervention had to result in at least $75 \%$ of products being offered required to be favourable or moderately favourable. As long as the $75 \%$ criterion was met, foods were not necessarily replaced by the same type of product. Control schools kept the original products.

\section{Number of comparisons analysed: 1}

Comparisons analysed: More unfavourable, high-energy extra food options available in vending machines versus fewer unfavourable, high-energy extra food options available in vending machines

\section{Concurrent intervention components in factorial design: No}

Concurrent intervention components confounded with comparison of interest: No

\section{Outcomes}

Outcomes reported in study: Mean proportions (\%) of products sold per product group and category. Mean sales volumes per food category averaged for number of students per school.

Selection outcome analysed: Mean proportion (\%) of unfavourable "extra" products (> $170 \mathrm{kcal}$ ) sold

Measurement of selection outcome: Objective

Timing of selection outcome measurement: Longer term (> 1 day)

Consumption outcome analysed: N/A

Measurement of consumption outcome: N/A

Timing of consumption outcome measurement: N/A

Secondary outcome: N/A

Funding source The Netherlands Organisation for Health Research and Development. The authors did not report on conflicts of interest. availability of higher-calorie products). In Phase II, labels were introduced, and in Phase III, prices were reduced. Purchase of extra products was selected as outcome measure as these were the least-nutritious categorisation of foods "that deliver only energy and no important vitamins or minerals" and relative importance of reducing consumption of high-energy products versus increasing consumption of low-energy products.

\begin{tabular}{ll}
\hline Methods & Study design: Between-participants randomised controlled trial \\
\hline Participants & Setting: Laboratory setting \\
& Setting type: Naturalistic laboratory (buffet installation within laboratory) \\
& Geographical region: Denmark \\
& Number of enrolled participants: 65 \\
& Number (\%) of enrolled participants completing the study and included in analysis: $100 \%$ \\
& Study completers - mean age (SD): $24.1(0.5)$ \\
& Study completers - sex: $100 \%$ male
\end{tabular}


Socioeconomic status context: Low deprivation

Inclusion criteria: Male university students aged between 18 and 29

Exclusion criteria: None reported

Type of proximity intervention: Proximity from start of buffet line (order encountered along line (e.g. queue or aisle))

\section{Manipulated product type: Food}

Characteristics of manipulated products: Mixed salad (comprising spinach, cauliflower, carrot, white cabbage, peas, apples, green beans, and parsley components) (in context of manipulation of other items in buffet order: pasta, bread, meatballs)

\section{Duration of exposure to intervention: $\leq 1$ day}

Study arms: Mixed salad placed at beginning of buffet queue line (with components in individual bowls); mixed salad placed later in buffet queue line (with components mixed in a single bowl)

\section{Number of comparisons analysed: 1}

Comparisons analysed: Mixed salad placed at start of buffet line, meaning accessed first versus mixed salad placed later in buffet line, meaning accessed after other foods

\section{Concurrent intervention components in factorial design: No}

Concurrent intervention components confounded with comparison of interest: Yes (salad components placed in individual bowls when mixed salad placed at start of buffet queue line, whilst salad components mixed in a single bowl when mixed salad placed at end of buffet queue line)

Outcomes

Outcomes reported in study: Self-served salad (fruit and vegetables); self-served pasta; self-served meatballs; self-served bread; total grams of all food served; total energy $(\mathrm{kJ})$ of all food served

Selection outcome analysed: Self-served salad (fruit and vegetables)

Measurement of selection outcome: Objective

Timing of selection outcome measurement: Immediate ( $\leq 1$ day)

Consumption outcome analysed: N/A

Measurement of consumption outcome: N/A

Timing of consumption outcome measurement: N/A

Secondary outcome: Total food (grams) selected; total energy (kJ) selected

Notes

Langlet 2017

Methods Study design: Between-participants cluster-randomised controlled trial

Participants

Setting: Laboratory setting 
Setting type: A room set up with tables and chairs (workstations) in a high school

Geographical region: Sweden

Number of enrolled participants: 41 ( 2 classes)

Number (\%) of enrolled participants completing the study and included in analysis: $41(100 \%)$

Study completers - mean age (SD): 16.8 years $(0.3)$ in the proximal group and $16.6(0.4)$ in the distal group

Study completers - sex: $54 \%$ female

Specific social or cultural characteristics: High school students between 15 and 17 years old who were attending a natural science class

Socioeconomic status context: Low deprivation

Inclusion criteria: Participants were recruited from 2 first-year natural science classes of a high school situated in central Stockholm (as part of a bigger trial organised in the school through the EU project SPLENDID, with the same student sample participating in behaviourally monitored lunch sessions earlier during the test days). Participation was non-discriminative, since every student was allowed to participate irrespective of their background, BMI, or sex.

Exclusion criteria: None reported

Interventions Intervention type: Proximity

Type of proximity intervention: Proximity from positioned participant

Manipulated product type: Food

Characteristics of manipulated products: Snack foods of grapes, chocolate, and crackers

Duration of exposure to intervention: $\leq 1$ day

Study arms: Proximal arm, where food was situated at arm's length from participants, and a distal arm, where food was situated at least $6 \mathrm{~m}$ away from participants

Number of comparisons analysed: 1

Comparisons analysed: Snack foods placed closer to participants versus snack foods placed farther away from participants

Concurrent intervention components in factorial design: No

Concurrent intervention components confounded with comparison of interest: No

Outcomes

Outcomes reported in study: Mean energy intake of each food type per participant, and the total energy intake per participant (for all snacks) (kcal); mean number of servings per individual across food types; mean energy content of each serving across food types (kcal)

Selection outcome analysed: Mean energy selected per individual (for all snacks) (kcal) (not directly reported in full but received from authors)

Measurement of selection outcome: Objective

Timing of selection outcome measurement: Immediate ( $\leq 1$ day)

Consumption outcome analysed: Mean energy intake per participant (for all snacks) (kcal)

Measurement of consumption outcome: Objective

Timing of consumption outcome measurement: Immediate ( $<1$ day) 
Langlet 2017 (Continued)

Secondary outcome: Mean energy content of each serving across food types (kcal); temporal analysis of servings

Funding source Internationella Engelska Gymnasiet SoÈdermalm (Internationella Engelska Skolan) and Division of Applied Neuroendocrinology (Department of Neurobiology, Care Sciences and Society, Karolinska Institutet) received funding from the European Community's Information and Communication Technology Programme under Grant Agreement No. 610746, 01/10/2013 $\pm 30 / 09 /$.

Notes

Selection outcome of mean energy selected per individual (for all snacks) (kcal) was not directly reported in full but was received from authors (26 November 2018) (as well as available in datasets posted on website of journal hosting publication although not in required form).

Maas 2012 (S1)

\begin{tabular}{ll}
\hline Methods & Study design: Between-participants randomised controlled trial \\
\hline Participants & Setting: Laboratory setting \\
Setting type: Laboratory & Geographical region: The Netherlands \\
& Number of enrolled participants: 80 \\
& Number (\%) of enrolled participants completing the study and included in analysis: 77 (96.3\%) \\
& Study completers - mean age (SD): 22.3 (3.7) \\
& Study completers - sex: $100 \%$ female \\
& Specific social or cultural characteristics: Female students \\
& Socioeconomic status context: Low deprivation \\
& Inclusion criteria: Female students; age 17 to 38 years; BMI between 18 and $30 \mathrm{~kg} / \mathrm{m}^{2}$ \\
& Exclusion criteria: Food allergies; current eating pathology
\end{tabular}

Type of proximity intervention: Proximity from positioned participant (chair at which seated) (distance from set point)

\section{Manipulated product type: Food}

Characteristics of manipulated products: Chocolate M\&Ms

Duration of exposure to intervention: $\leq 1$ day

Study arms: $1000 \mathrm{~g}$ of chocolate M\&Ms in a transparent bowl placed $20 \mathrm{~cm}$ from seated participants' right armrest; $1000 \mathrm{~g}$ of chocolate M\&Ms in a transparent bowl placed $70 \mathrm{~cm}$ from seated participants' right armrest; $1000 \mathrm{~g}$ of chocolate M\&Ms placed $140 \mathrm{~cm}$ from seated participants' right armrest

\section{Number of comparisons analysed: 2}

\section{Comparisons analysed:}

Comparison 1: M\&Ms placed $20 \mathrm{~cm}$ from participant versus M\&Ms placed $70 \mathrm{~cm}$ from participant

Comparison 2: M\&Ms placed $20 \mathrm{~cm}$ from participant versus M\&Ms placed $140 \mathrm{~cm}$ from participant

\section{Concurrent intervention components in factorial design: No}


Maas 2012 (S1) (Continued)

Concurrent intervention components confounded with comparison of interest: No

\begin{tabular}{l} 
Outcomes reported in study: Likelihood of snack consumption; amount of snacks consumed \\
Selection outcome analysed: N/A \\
Measurement of selection outcome: N/A \\
Timing of selection outcome measurement: N/A \\
Consumption outcome analysed: Amount of snacks consumed \\
Measurement of consumption outcome: Objective \\
Timing of consumption outcome measurement: Immediate ( $\leq 1$ day) \\
Secondary outcome: N/A \\
Funding and conflicts of interest were not reported. \\
\hline Funding source \\
Amount of snacks consumed was the selected outcome of interest because the amount consumed is \\
more relevant to health outcomes than whether any were consumed.
\end{tabular}

Maas 2012 (S2)

\begin{tabular}{ll}
\hline Methods & Study design: Between-participants randomised controlled trial \\
\hline Participants & Setting: Laboratory setting \\
Setting type: Laboratory & Geographical region: The Netherlands \\
& Number of enrolled participants: 58 \\
& Number (\%) of enrolled participants completing the study and included in analysis: 54 (93.1\%) \\
& Study completers - mean age (SD): 21.3 (2.6) \\
& Study completers - sex: $100 \%$ female \\
& Specific social or cultural characteristics: Female students \\
& Socioeconomic status context: Low deprivation \\
& Inclusion criteria: Female students; age 17 to 29 years; BMI between 18 and $30 \mathrm{~kg} / \mathrm{m}^{2}$ \\
& Exclusion criteria: Food allergies; current eating pathology
\end{tabular}

Interventions

Intervention type: Proximity

Type of proximity intervention: Proximity from positioned participant (chair at which seated) (distance from set point)

Manipulated product type: Food

Characteristics of manipulated products: Chocolate M\&Ms

Duration of exposure to intervention: $\leq 1$ day 
Study arms: $1000 \mathrm{~g}$ of chocolate M\&Ms in a transparent bowl placed $20 \mathrm{~cm}$ from seated participants' right armrest; $1000 \mathrm{~g}$ of chocolate M\&Ms in a transparent bowl placed $70 \mathrm{~cm}$ from seated participants' right armrest; $1000 \mathrm{~g}$ of chocolate M\&Ms placed $140 \mathrm{~cm}$ from seated participants' right armrest

\section{Number of comparisons analysed: 2}

\section{Comparisons analysed:}

Comparison 1: M\&Ms placed $20 \mathrm{~cm}$ from participant versus M\&Ms placed $70 \mathrm{~cm}$ from participant

Comparison 2: M\&Ms placed $20 \mathrm{~cm}$ from participant versus M\&Ms placed $140 \mathrm{~cm}$ from participant

Concurrent intervention components in factorial design: No

Concurrent intervention components confounded with comparison of interest: N/A

\begin{tabular}{l} 
Outcomes reported in study: Likelihood of snack consumption; amount of snacks consumed \\
Selection outcome analysed: N/A \\
Measurement of selection outcome: N/A \\
Timing of selection outcome measurement: N/A \\
Consumption outcome analysed: Amount of snacks consumed \\
Measurement of consumption outcome: Objective \\
Timing of consumption outcome measurement: Immediate ( 1 day) \\
Secondary outcome: N/A \\
Funding and conflicts of interest were not reported. \\
\hline Funding source \\
Amount of snacks consumed was the selected outcome of interest because the amount consumed is \\
more relevant to health outcomes than whether any were consumed.
\end{tabular}

Musher-Eizenman 2010

Methods Study design: Between-participants randomised controlled trial

Participants Setting: Laboratory setting

Setting type: Naturalistic laboratory (dining room set up in a school gymnasium)

Geographical region: USA

Number of enrolled participants: 31

Number (\%) of enrolled participants completing the study and included in analysis: 27 (87.1\%)

Study completers - mean age (SD): $6.3(2.3)$

Study completers - sex: $42 \%$ female

Specific social or cultural characteristics: Preschool and school-age children at a local childcare centre

Socioeconomic status context: Low deprivation

Inclusion criteria: Children agreeing to take part in the snack time activity, agreeing to answer questions from the researchers, and agreeing to be weighed and measured 
Exclusion criteria: None reported

Interventions

Intervention type: Proximity

Type of proximity intervention: Proximity from positioned participant (table at which seated) (distance from set point)

\section{Manipulated product type: Food}

Characteristics of manipulated products: Animal crackers (cookies) (in context of also manipulating carrot slices on subsequent testing session)

Duration of exposure to intervention: $\leq 1$ day

Study arms: Seated at Table 1 (nearest to snack bowl); Seated at Table 2; Seated at Table 3; Seated at Table 4; Seated at Table 5 (farthest from snack bowl)

Number of comparisons analysed: 1

Comparisons analysed:

Comparison 1: Participants seated at Tables 1 and 2 (i.e. serving bowl of animal crackers placed nearest $(28 \mathrm{~cm}$ ) to table (participant seated at Table 1$)$ plus serving bowl of animal crackers placed approximately $250 \mathrm{~cm}$ to table (participant seated at Table 2)); participants seated at Tables 3, 4, and 5 (i.e. serving bowl of animal crackers placed approximately $500 \mathrm{~cm}$ to table (participant seated at Table 3) plus serving bowl of animal crackers placed approximately $750 \mathrm{~cm}$ to table (participant seated at Table 4) plus serving bowl of animal crackers placed approximately $1000 \mathrm{~cm}$ to table (participant seated at Table 5))

Concurrent intervention components in factorial design: No

Concurrent intervention components confounded with comparison of interest: N/A

Outcomes

Outcomes reported in study: Mean number of animal crackers consumed; mean number of carrot slices consumed

Selection outcome analysed: N/A

Measurement of selection outcome: N/A

Timing of selection outcome measurement: N/A

Consumption outcome analysed: Mean number of animal crackers consumed

Measurement of consumption outcome: Objective

Timing of consumption outcome measurement: Immediate ( $\leq 1$ day)

Secondary outcome: N/A

Funding source $\quad$ Funding and conflicts of interest were not reported.

Notes Attempted to contact authors April 2017 and November 2017 to confirm outcome data and if participants re-randomised on each of the 2 testing days, but no response received. Cannot assume re-randomisation, so treated as single study.

Painter 2002

\begin{tabular}{ll}
\hline Methods & Study design: Within-participants cross-over randomised controlled trial \\
\hline Participants & Setting: Field setting
\end{tabular}


Painter 2002 (Continued)

Setting type: Workplace office in university setting

Geographical region: USA

Number of enrolled participants: 16

Number (\%) of enrolled participants completing the study and included in analysis: $16(100 \%)$

Study completers - mean age (SD): Not reported (median $=43$ years)

Study completers - sex: $62.5 \%$ female

Specific social or cultural characteristics: Office workers at university workplace office

Socioeconomic status context: Low deprivation

Inclusion criteria: Office workers at a university workplace office

Exclusion criteria: None reported

Interventions

Intervention type: Proximity

Type of proximity intervention: Proximity from positioned participant (desk at which seated) (distance from set point)

Manipulated product type: Food

Characteristics of manipulated products: Chocolate (candy chocolate "kisses" in a container)

Duration of exposure to intervention: $>1$ day

Study arms: Chocolate placed on top of the desk (near and visible); chocolate placed in desk drawer (near but not visible); chocolate placed on distal shelf ( $200 \mathrm{~cm}$ ( $2 \mathrm{~m})$ away) (far and visible)

Number of comparisons analysed: 1

Comparisons analysed: Chocolate placed on top of the desk (near and visible) versus chocolate placed on distal shelf ( $200 \mathrm{~cm}(2 \mathrm{~m})$ away) (far and visible)

Concurrent intervention components in factorial design: No

Concurrent intervention components confounded with comparison of interest: N/A

\begin{tabular}{|c|c|}
\hline Outcomes & $\begin{array}{l}\text { Outcomes reported in study: Mean number of chocolates consumed per day } \\
\text { Selection outcome analysed: N/A } \\
\text { Measurement of selection outcome: N/A } \\
\text { Timing of selection outcome measurement: N/A } \\
\text { Consumption outcome analysed: Mean number of chocolates consumed per day } \\
\text { Measurement of consumption outcome: Objective } \\
\text { Timing of consumption outcome measurement: Longer term (> 1 day) } \\
\text { Secondary outcome: N/A }\end{array}$ \\
\hline Funding source & Funding and conflicts of interest were not reported. \\
\hline Notes & $\begin{array}{l}\text { The corresponding author confirmed that assignment was randomised ( } 16 \text { March 2016). Outcome data } \\
\text { (standard deviations) requested from the authors in November } 2017 \text { were not received. The study arm } \\
\text { in which chocolates were placed in desk drawer (so as to not be visible) was excluded from our analy- } \\
\text { ses as it confounds the effect of proximity and is not relevant to the current review. }\end{array}$ \\
\hline
\end{tabular}

Altering the availability or proximity of food, alcohol, and tobacco products to change their selection and consumption (Review) 
Pechey 2019

Methods

Participants
Study design: Stepped-wedge cluster-randomised controlled trial

Setting: Field setting

Setting type: Workplace cafeterias

Geographical region: UK

Number of enrolled participants: Not reported (there were potentially 5200 employees targeted from 6 study sites)

Number (\%) of enrolled participants completing the study and included in analysis: 6 sites (100\%)

Study completers - mean age (SD): Not reported

Study completers - sex: Not reported

Specific social or cultural characteristics: Employees at the study sites included: those with higher and intermediate managerial, administrative, and professional occupations; those with supervisory, clerical, and junior managerial, administrative, and professional occupations; and those with semi-skilled and unskilled manual occupations

Socioeconomic status context: Low deprivation

Inclusion criteria: Sites were included if they: (a) were based in England, (b) had approximately 350 or more employees, and (c) could provide at least weekly sales data on individual items and the energy (kcal) content of items sold

Exclusion criteria: None reported
Intervention type: Availability

Type of availability intervention: Relative number (proportion) of less-healthy (to healthier) options: Higher and lower availability of less-healthy (i.e. higher-energy) cooked meal, snack, cold drink, and sandwich options (relative to healthier (i.e. lower-energy) options)

\section{Manipulated product type: Food}

Characteristics of manipulated products: Less-healthy foods replaced by healthier cooked meals, sandwiches, and snacks: healthier cooked meals (excluding breakfast) were defined as having under $300 \mathrm{kcal}$ for a meal component or under $500 \mathrm{kcal}$ for a complete meal; healthier sandwiches (or equivalents, e.g. wraps, panini, baguettes, bagels) were defined as those under $350 \mathrm{kcal}$; healthier savoury snacks were: under 120 kcal per pack; sweet snacks: under 150 kcal per pack; and cold drinks: under 50 kcal per pack (e.g. zero or light varieties)

\section{Duration of exposure to intervention: $\geq 1$ day}

Study arms: No changes made in cafeterias; cafeterias with reductions in less-healthy food and increased healthy foods (without changing the absolute number of food items offered)

\section{Number of comparisons analysed: 1}

Comparisons analysed: Baseline data/no change in food items offered in cafeterias (higher availability of less-healthy food) versus lower availability of less-healthy food and increased availability of healthy cooked meals, sandwiches, and snacks

\section{Concurrent intervention components in factorial design: No}

\section{Concurrent intervention components confounded with comparison of interest:}

No 
Pechey 2019 (Continued)

Outcomes
Outcomes reported in study: Total energy (kcal) purchased per day from intervention categories; total energy ( $\mathrm{kcal}$ ) purchased per day from all categories; total revenue (GBP) per day from all categories

Selection outcome analysed: Total energy (kcal) purchased per day from intervention categories

Measurement of selection outcome: Objective

Timing of selection outcome measurement: Longer term (> 1 day)

Consumption outcome analysed: N/A

Measurement of consumption outcome: N/A

Timing of consumption outcome measurement: $N / A$

Secondary outcome: Total energy (kcal) purchased per day from all categories; total revenue (GBP) per day from all categories
Funding source

Funded by the National Institute for Health Research Policy Research Programme (Policy Research Unit in Behaviour and Health (PR-UN-0409-10109) and IGD (Institute of Grocery Distribution) (RG83425)). The authors stated that there were no conflicts of interest.

Notes

Trial registration record at clinicaltrials.gov/ct2/show/nct03252158. Cluster-randomised trial, but data were aggregate/cluster level. During the intervention period, sites were asked to position replacements in the same location, and with as close as possible to the same number of packs as the removed product, and to restock these as usual. Replacement products were priced at their recommended retail price or using the catering providers' normal pricing guidance. Statistical data necessary to input SMD and 95\% Cls were provided by study authors in November 2018.

Privitera 2012 (S1)

\begin{tabular}{|c|c|}
\hline Methods & Study design: Between-participants randomised controlled trial \\
\hline \multirow[t]{11}{*}{ Participants } & Setting: Laboratory setting \\
\hline & Setting type: Naturalistic laboratory (kitchen space) \\
\hline & Geographical region: USA \\
\hline & Number of enrolled participants: 48 \\
\hline & Number (\%) of enrolled participants completing the study: No information \\
\hline & Study completers - mean age (SD): 19.9 (1.1) \\
\hline & Study completers - sex: Not reported \\
\hline & Specific social or cultural characteristics: University students \\
\hline & Socioeconomic status context: Low deprivation \\
\hline & Inclusion criteria: None reported - recruitment via classroom visits \\
\hline & Exclusion criteria: Participants who had eaten in the 2 hours prior to start of the study were excluded \\
\hline \multirow[t]{3}{*}{ Interventions } & Intervention type: Proximity \\
\hline & $\begin{array}{l}\text { Type of proximity intervention: Proximity from participant (kitchen table at which seated) (distance } \\
\text { from set point) }\end{array}$ \\
\hline & Manipulated product type: Food \\
\hline
\end{tabular}


Privitera 2012 (S1) (Continued)

Characteristics of manipulated products: Sweet red apple slices

Duration of exposure to intervention: $\leq 1$ day

Study arms: On table, clear bowl; on counter, clear bowl; on table, opaque bowl; on counter, opaque bowl

Number of comparisons analysed: 1

Comparisons analysed: Apple slices in bowl on table (within arm's reach) versus bowl on counter (200 $\mathrm{cm}$ away)

Concurrent intervention components in factorial design: Yes (opacity of bowl container was manipulated to alter food visibility)

Concurrent intervention components confounded with comparison of interest: No

Outcomes reported in study: Number of items consumed

Selection outcome analysed: N/A

Measurement of selection outcome: N/A

Timing of selection outcome measurement: N/A

Consumption outcome analysed: Number of items consumed

Measurement of consumption outcome: Objective

Timing of consumption outcome measurement: Immediate ( $\leq 1$ day)

Secondary outcome: N/A

Notes

Methods Study design: Between-participants randomised controlled trial

Participants

\author{
Setting: Laboratory setting \\ Setting type: Naturalistic laboratory (kitchen space) \\ Geographical region: USA \\ Number of enrolled participants: 48 \\ Number (\%) of enrolled participants completing the study: No information \\ Study completers - mean age (SD): 20.1 (1.6) \\ Study completers - sex: Not reported \\ Specific social or cultural characteristics: University students \\ Socioeconomic status context: Low deprivation \\ Inclusion criteria: None reported - recruitment via classroom visits \\ Exclusion criteria: Participants who had eaten in the 2 hours prior to start of the study were excluded
}


Privitera 2012 (S2) (Continued)

Interventions

Intervention type: Proximity

Type of proximity intervention: Proximity from participant (kitchen table at which seated) (distance from set point)

Manipulated product type: Food

Characteristics of manipulated products: Baby-cut carrots

Duration of exposure to intervention: $\leq 1$ day

Study arms: On table, clear bowl; on counter, clear bowl; on table, opaque bowl; on counter, opaque bowl

Number of comparisons analysed: 1

Comparisons analysed: Baby-cut carrots in bowl on table (within arm's reach) versus bowl on counter (200 cm away)

Concurrent intervention components in factorial design: Yes (opacity of bowl container was manipulated to alter food visibility)

Concurrent intervention components confounded with comparison of interest: No

Outcomes reported in study: Number of items consumed

Selection outcome analysed: N/A

Measurement of selection outcome: N/A

Timing of selection outcome measurement: N/A

Consumption outcome analysed: Number of items consumed

Measurement of consumption outcome: Objective

Timing of consumption outcome measurement: Immediate ( $\leq 1$ day)

Secondary outcome: N/A

Notes

Privitera 2014

Methods Study design: Between-participants randomised controlled trial

Participants

Setting: Laboratory setting

Setting type: Naturalistic laboratory (kitchen space)

Geographical region: USA

Number of enrolled participants: 60

Number (\%) of enrolled participants completing the study: 56 (93.3\%)

Study completers - mean age (SD): 19.4 (0.9)

Study completers - sex: $53.6 \%$ female 
Privitera 2014 (Continued)

Specific social or cultural characteristics: University students

Socioeconomic status context: Low deprivation

Inclusion criteria: None reported - recruitment via classroom visits

Exclusion criteria: Participants who had eaten in the 2 hours prior to start of the study were excluded

Interventions

Intervention type: Proximity

Type of proximity intervention: Proximity from participant (kitchen table at which seated) (distance from set point)

Manipulated product type: Food

Characteristics of manipulated products: Butter popcorn (high-calorie food (158 calories and $734 \mathrm{~kJ}$ per serving) in context of also a proximity manipulation of lower-calorie food, apple slices ( 0 calories and energy density is $304 \mathrm{~kJ}$ per serving))

Duration of exposure to intervention: $\leq 1$ day

Study arms: Apple near $(30 \mathrm{~cm})$, popcorn far $(200 \mathrm{~cm})$; popcorn near, apple far; both near

Number of comparisons analysed: 1

Comparisons analysed: Popcorn near (apple near) versus popcorn far (apple near)

Concurrent intervention components in factorial design: No

Concurrent intervention components confounded with comparison of interest: No (location of a secondary non-target food was changed, but selected comparisons of interest for review unaffected)

\begin{tabular}{ll} 
Outcomes & Outcomes reported in study: Popcorn consumption; apple consumption \\
Selection outcome analysed: N/A & Measurement of selection outcome: N/A \\
& Timing of selection outcome measurement: N/A \\
& Consumption outcome analysed: Popcorn consumption (g) \\
& Measurement of consumption outcome: Objective \\
& Timing of consumption outcome measurement: Immediate ( $\leq 1$ day) \\
& Secondary outcome: N/A \\
\hline Funding source & Funding and conflicts of interest were not reported. \\
\hline Notes & \\
\hline
\end{tabular}

\begin{tabular}{ll}
\hline Methods & Study design: Within-participants cross-over cluster-randomised controlled trial \\
\hline Participants & Setting: Field setting \\
& Setting type: Childcare facility \\
Geographical region: USA
\end{tabular}


Roe 2013 (Continued)

\author{
Number of enrolled participants: 61 (4 classrooms) \\ Number (\%) of enrolled participants completing the study: $61(100 \%)$ \\ Study completers - mean age (SD): 4.4 (0.5) \\ Study completers - sex: $52.5 \%$ female
}

Specific social or cultural characteristics: Schoolchildren

Socioeconomic status context: Low deprivation

Inclusion criteria: Not stated, children aged 3 to 5 years

Exclusion criteria: Not stated, children who were allergic to any of the foods served were not included

Type of availability intervention: Absolute number of different options available: higher and lower availability of fruits and vegetables (children offered 3 varieties of fruit or vegetable or a single type of fruit or vegetable)

\title{
Manipulated product type: Food
}

Characteristics of manipulated products: Fruit (apple, peach, pineapple) and vegetable (cucumber, sweet potato, tomato) snacks. All children were also provided with a small piece of pita bread (16 g; 43 $\mathrm{kcal}$ ) and $250 \mathrm{~mL}$ of water.

\section{Duration of exposure to intervention: $>1$ day}

Study arms: Children were offered 3 varieties of fruit or vegetables from a bowl; children were offered a single type of fruit or vegetable from a bowl

Number of comparisons analysed: 1

Comparisons analysed: 3 types of fruit or vegetable versus 1 type of fruit or vegetable

Concurrent intervention components in factorial design: No

Concurrent intervention components confounded with comparison of interest: N/A

Outcomes reported in study: Mean number of fruit and vegetable pieces selected; mean number of fruit and vegetable pieces eaten

Selection outcome analysed: Mean number of fruit and vegetable pieces selected

Measurement of selection outcome: Objective

Timing of selection outcome measurement: Longer term (> 1 day)

Consumption outcome analysed: Mean number of fruit and vegetable pieces eaten

Measurement of consumption outcome: Objective

Timing of consumption outcome measurement: Longer term (> 1 day)

Secondary outcome: N/A 


\begin{tabular}{ll}
\hline Methods & Study design: Within-participants cross-over randomised controlled trial \\
\hline Participants & Setting: Laboratory setting \\
Setting type: Laboratory (Human Nutrition Unit, where participants had their own fridge, freezer, and \\
microwave) \\
Geographical region: UK \\
Number of enrolled participants: 12 \\
Number (\%) of enrolled participants completing the study and included in analysis: 12 (100\%) \\
Study completers - mean age (SD): 33.4 (7.2) \\
Study completers - sex: $100 \%$ male \\
Specific social or cultural characteristics: Men only \\
Socioeconomic status context: Low deprivation \\
Inclusion criteria: Not stated, healthy, non-smoking men \\
Exclusion criteria: Not stated, men who were following a specialised diet or exercise regimen
\end{tabular}
Intervention type: Availability

Type of availability intervention: Absolute number of different options available: higher and lower variety of foods (number of foods provided on a daily menu)

\section{Manipulated product type: Food}

Characteristics of manipulated products: Breakfast, lunch, dinner meals, and snacks

Duration of exposure to intervention: > 1 day

Study arms: Participants were studied for three 9-day periods. From days 3 to 9, participants were given ad libitum access to a 3-day rotating menu of either low-, medium-, or high-variety foods. Study arms were: low variety, with 5 items available; medium variety, with 10 items available; high variety, with 15 items available.

\section{Number of comparisons analysed: 2}

\section{Comparisons analysed:}

Comparison 1: high-variety arm (for meals, participants had 3 menu options) versus medium-variety arm (for meals, participants had 2 menu options). Each food item had a similar macronutrient composition.

Comparison 2: medium-variety arm (for meals, participants had 2 menu options) versus low-variety arm (for meals, participants had 1 menu option). Each food item had a similar macronutrient composition.

\section{Concurrent intervention components in factorial design: No}

Concurrent intervention components confounded with comparison of interest: N/A

Outcomes

Outcomes reported in study: Average daily food, energy, and macronutrient intakes (weight, energy, protein, fat, carbohydrate)

\section{Selection outcome analysed: N/A}

\section{Measurement of selection outcome: N/A}

Timing of selection outcome measurement: N/A 
Stubbs 2001 (Continued)

Consumption outcome analysed: Energy intake (MJ)

Measurement of consumption outcome: Objective

Timing of consumption outcome measurement: Long term (> 1 day)

Secondary outcome: N/A

\begin{tabular}{ll}
\hline Funding source & The work was supported by the Scottish Office. No conflicts of interest were reported. \\
\hline Notes & Outcome data (standard deviations) requested from the authors in November 2017 were not received. \\
\hline
\end{tabular}

Wansink 2006

Methods Study design: Within-participants cross-over randomised controlled trial

Participants

Setting: Field setting

Setting type: Workplace offices in university setting

Geographical region: USA

Number of enrolled participants: 40

Number (\%) of enrolled participants completing the study and included in analysis: 40 (100\%)

Study completers - mean age (SD): 42.2 (11.3)

Study completers - sex: $100 \%$ female

Specific social or cultural characteristics: Office workers at university workplace

Socioeconomic status context: Low deprivation

Inclusion criteria: Female office workers in a university setting; typically consume 3 or more pieces of candy each week

Exclusion criteria: Those who eat less than 3 pieces of candy a week

Type of proximity intervention: Proximity from positioned participant (desk at which seated) (distance from set point)

\section{Manipulated product type: Food}

Characteristics of manipulated products: Chocolate (candy chocolate "kisses")

Duration of exposure to intervention: $>1$ day

Study arms: Chocolate placed in covered clear bowl on top of desk; chocolate placed in covered opaque bowl on top of desk; chocolate placed in covered clear bowl $200 \mathrm{~cm}(2 \mathrm{~m})$ away from desk at similar level; chocolate placed in covered opaque bowl $200 \mathrm{~cm}(2 \mathrm{~m})$ away from desk at similar level

\section{Number of comparisons analysed: 1}

Comparisons analysed: Chocolate placed in bowl on top of desk versus chocolate placed in bowl 200 $\mathrm{cm}(2 \mathrm{~m})$ away from desk at similar level

Concurrent intervention components in factorial design: Yes (clear or opaque bowl)

Concurrent intervention components confounded with comparison of interest: No 
Wansink 2006 (Continued)

Outcomes reported in study: Mean number of chocolates consumed per day

Selection outcome analysed: N/A

Measurement of selection outcome: N/A

Timing of selection outcome measurement: N/A

Consumption outcome analysed: Mean number of chocolates consumed per day

Measurement of consumption outcome: Objective

Timing of consumption outcome measurement: Longer term ( $>1$ day)

Secondary outcome: N/A

Funding source $\quad$ Funding and conflicts of interest were not reported.

Notes Author confirmed that assignment was randomised (16 March 2016). Outcome data (standard deviations) requested from the authors in November 2017 were not received.

Wansink 2013a

\begin{tabular}{ll}
\hline Methods & Study design: Between-participants randomised controlled trial \\
\hline Participants & Setting: Field setting
\end{tabular}

Participants

Setting type: Breakfast buffet at work conference

Geographical region: USA

Number of enrolled participants: 124

Number (\%) of enrolled participants completing the study and included in analysis: 124 (100\%)

Study completers - mean age (SD): Not reported

Study completers - sex: Not reported

Specific social or cultural characteristics: Human Resource managers attending a conference on behaviour change and health

Socioeconomic status context: Low deprivation

Inclusion criteria: Human Resource managers attending a conference on behaviour change and health

Exclusion criteria: None reported

Type of proximity intervention: Proximity from start of queue line (start or end of a buffet line)

Manipulated product type: Food

Characteristics of manipulated products: Cheesy eggs (in context of also proximity manipulation of other items in buffet order: fried potatoes, bacon, cinnamon rolls, low-fat granola, low-fat yoghurt, fruit)

\section{Duration of exposure to intervention: $\leq 1$ day}


Study arms: Least-healthy food (cheesy eggs) placed first in buffet line, amongst set of foods placed in order of less healthy to healthier; least-healthy food (cheesy eggs) placed last in buffet line, amongst set of foods placed in order of healthier to less healthy

\section{Number of comparisons analysed: 1}

Comparisons analysed: Least-healthy food (cheesy eggs) placed first in buffet line, amongst set of foods placed in order of less healthy to healthier versus least-healthy food (cheesy eggs) placed last in buffet line, amongst set of foods placed in order of healthier to less healthy

\section{Concurrent intervention components in factorial design: No}

Concurrent intervention components confounded with comparison of interest: N/A

Outcomes

Outcomes reported in study: Proportion of participants selecting least-healthy food (cheesy eggs); proportion of participants selecting fried potatoes; proportion of participants selecting bacon; proportion of participants selecting cinnamon rolls; proportion of participants selecting low-fat granola; proportion of participants selecting low-fat yoghurt; proportion of participants selecting fruit

Selection outcome analysed: Proportion of participants selecting least-healthy food (cheesy eggs)

Measurement of selection outcome: Objective

Timing of selection outcome measurement: Immediate ( $\leq 1$ day)

Consumption outcome analysed: N/A

Measurement of consumption outcome: N/A

Timing of consumption outcome measurement: N/A

Secondary outcome: N/A

Funding source The authors stated that no support or funding was received, and that there were no conflicts of interest.

Notes

Data were modelled, and the raw data based on observations were not obtainable from the authors (contacted November 2017). This study was therefore excluded from the analysis.

BMI: body mass index

kcal: kilocalories

N/A: not applicable

SD: standard deviation

Characteristics of excluded studies [ordered by study ID]

\begin{tabular}{ll}
\hline Study & Reason for exclusion \\
\hline Alinia 2011 & Study design ineligible \\
\hline Altintzoglou 2015 & No measurement of selection or consumption \\
\hline Anderson 2005 & No eligible intervention \\
\hline Angelopoulos 2009 & No eligible intervention \\
\hline Ayala 2013 & No eligible intervention \\
\hline Ayala 2015 & No eligible intervention \\
\hline
\end{tabular}

Altering the availability or proximity of food, alcohol, and tobacco products to change their selection and consumption (Review) 


\begin{tabular}{|c|c|}
\hline Study & Reason for exclusion \\
\hline Backman 2011 & No eligible intervention \\
\hline Bartholomew 2006 & Study design ineligible \\
\hline Beets 2014 & No measurement of selection or consumption \\
\hline Bere 2006 & No eligible intervention \\
\hline Bergamaschi 2016 & No eligible intervention \\
\hline Bonsergent 2013 & No eligible intervention \\
\hline Bonvecchio-Arenas 2010 & No eligible intervention \\
\hline Bucher 2011 & No eligible intervention \\
\hline Bucher 2014 & No eligible intervention \\
\hline Burns 2015 & No eligible intervention \\
\hline Butler 1996 & No measurement of selection or consumption \\
\hline Caldwell 2009 & No eligible intervention \\
\hline Carroll 2018 & No measurement of selection or consumption \\
\hline Chandon 2002 & Study design ineligible \\
\hline Cohen 2014 & No eligible intervention \\
\hline Coleman 2012 & No eligible intervention \\
\hline Dayan 2011 & No eligible intervention \\
\hline De Wild 2015 & No eligible intervention \\
\hline Divert 2015 & Study design ineligible \\
\hline Dominguez 2013 & No eligible intervention \\
\hline Elsbernd 2016 & No eligible intervention \\
\hline Epstein 2009 & No measurement of selection or consumption \\
\hline Epstein 2013 & No eligible intervention \\
\hline Epstein 2015 & No eligible intervention \\
\hline Eriksen 2003 & No eligible intervention \\
\hline Fisher 1999 & No eligible intervention \\
\hline French 2004 & No eligible intervention \\
\hline French 2005a & No eligible intervention \\
\hline
\end{tabular}




\begin{tabular}{|c|c|}
\hline Study & Reason for exclusion \\
\hline French 2005b & No eligible intervention \\
\hline French 2010a & No eligible intervention \\
\hline French 2010b & No eligible intervention \\
\hline Giles 2012 & No eligible intervention \\
\hline Gillis 2009 & No eligible intervention \\
\hline Gittelsohn 2013 & No eligible intervention \\
\hline Hanks 2012 & Study design ineligible \\
\hline Harnack 2012 & No eligible intervention \\
\hline Haws 2013 & No eligible intervention \\
\hline Hoerr 1993 & Study design ineligible \\
\hline Kahn 2004 & No eligible intervention \\
\hline Keller 2015 & No measurement of selection or consumption \\
\hline Kim 2012 & Study design ineligible \\
\hline Kimathi 2009 & Study design ineligible \\
\hline Kingsland 2011 & No eligible intervention \\
\hline Kocken 2015 & No eligible intervention \\
\hline Kovalskys 2010 & No eligible intervention \\
\hline Kroese 2016 & Study design ineligible \\
\hline Lachat 2009 & No eligible intervention \\
\hline Leak 2014 & No eligible intervention \\
\hline Lee 2014 & Study design ineligible \\
\hline Lee-Kwan 2013 & Study design ineligible \\
\hline Linde 2012 & No eligible intervention \\
\hline Loughridge 2005 & Study design ineligible \\
\hline Lytle 2006 & No eligible intervention \\
\hline Marcus 2009 & No eligible intervention \\
\hline Martinez-Donate 2015 & No eligible intervention \\
\hline Meengs 2012 & No eligible intervention \\
\hline
\end{tabular}




\begin{tabular}{|c|c|}
\hline Study & Reason for exclusion \\
\hline Mennella 2008 & No measurement of selection or consumption \\
\hline Meyers 1980 & Study design ineligible \\
\hline Moore 2008 & No eligible intervention \\
\hline Muckelbauer 2009 & No eligible intervention \\
\hline Muckelbauer 2009b & No eligible intervention \\
\hline Norton 2006 & No measurement of selection or consumption \\
\hline Novotny 2011 & No eligible intervention \\
\hline O'Connell 2012 & No eligible intervention \\
\hline Parker 2001 & No eligible intervention \\
\hline Patel 2011 & No eligible intervention \\
\hline Perez-Morales 2011 & No eligible intervention \\
\hline Perry 2004 & No eligible intervention \\
\hline Rausch 2013 & No eligible intervention \\
\hline Raynor 2006 & No eligible intervention \\
\hline Raynor 2012a & No eligible intervention \\
\hline Raynor 2012b & No eligible intervention \\
\hline Raynor 2012c & No eligible intervention \\
\hline Redden 2015 & Study design ineligible \\
\hline Romero 2016 & No eligible intervention \\
\hline Rozin 2011 & Study design ineligible \\
\hline Sallis 2003 & No eligible intervention \\
\hline Scherr 2013 & No eligible intervention \\
\hline Scherr 2014 & No eligible intervention \\
\hline Schwartz 2009 & Study design ineligible \\
\hline Shin 2015 & No eligible intervention \\
\hline Sigurdsson 2011 & Study design ineligible \\
\hline Song 2008 & Study design ineligible \\
\hline Stea 2009 & Study design ineligible \\
\hline
\end{tabular}




\begin{tabular}{|c|c|}
\hline Study & Reason for exclusion \\
\hline Steenhuis 2004 & No eligible intervention \\
\hline Steeves 2015 & No eligible intervention \\
\hline Steyn 2015 & No eligible intervention \\
\hline Te Velde 2008 & No eligible intervention \\
\hline Temple 2008 & No eligible intervention \\
\hline Thorndike 2016 & No eligible intervention \\
\hline Thorndike 2017 & No eligible intervention \\
\hline Toft 2017 & Study design ineligible \\
\hline Uglem 2013 & Study design ineligible \\
\hline Uglem 2014 & Study design ineligible \\
\hline van Herpen 2014 & No eligible intervention \\
\hline van Kleef 2012 & Study design ineligible \\
\hline Van Lippevelde 2012 & No eligible intervention \\
\hline Visscher 2010 & Study design ineligible \\
\hline Wansink 2011 & Not an empirical study \\
\hline Wansink 2013b & No measurement of selection or consumption \\
\hline Wijnhoven 2015 & No eligible intervention \\
\hline Wilbur 1981 & Study design ineligible \\
\hline Wilcox 2009 & No eligible intervention \\
\hline Wilson 2017 & Study design ineligible \\
\hline Wolfenden 2015 & No eligible intervention \\
\hline Wordell 2012 & Study design ineligible \\
\hline Zeinstra 2010 & No eligible intervention \\
\hline
\end{tabular}

Characteristics of studies awaiting assessment [ordered by study ID]

Hua 2017

Methods $2 \times 2 \times 2$ factorial randomised controlled trial

Participants Students, staff, and employees on a university campus 
Hua 2017 (Continued)

Interventions
All co-located snack and beverage vending machines ( $n=56,28$ snack and 28 beverage) were randomised into 1 of 8 conditions (from a $2 \times 2 \times 2$ factorial design): higher availability of healthier products (or not) and/or $25 \%$ price reduction for healthier items (or not) and/or promotional signs on machines (or not).

\begin{tabular}{ll}
\hline Outcomes & The main outcome measures were changes in units sold and revenue. \\
\hline Notes & $\begin{array}{l}\text { Identified in updated searches in July 2018. Authors contacted 5th October } 2018 \text { for additional in- } \\
\text { formation as to eligibility and whether necessary data were collected but no response received. }\end{array}$ \\
\hline
\end{tabular}

\section{Watanabe 2016}

$\begin{array}{ll}\text { Methods } & \begin{array}{l}\text { Students were randomly divided into } 2 \text { groups for a buffet lunch in } 2 \text { separate rooms. In } 1 \text { room, } 16 \\ \text { kinds of foods were ordered, from vegetable foods to protein foods (vegetable-first group), whilst } \\ \text { the dishes were in reverse order in the other room (protein-first group). }\end{array}\end{array}$

\begin{tabular}{ll}
\hline Participants & 61 students \\
\hline Interventions & $\begin{array}{l}\text { Foods ordered from vegetable foods to protein foods (vegetable-first group) versus dishes in re- } \\
\text { verse order in other room (protein-first group) }\end{array}$
\end{tabular}

Outcomes Number of consumed foods was compared between the 2 groups.

Notes Authors contacted for full study report.

\section{Characteristics of ongoing studies [ordered by study ID]}

Knowles 2017

\section{Trial name or title}

Methods

\section{Participants}

Interventions

\section{Outcomes}

Starting date

Contact information

Daniel Knowles<Daniel.Knowles@bcu.ac.uk>

Detailed in registration on Open Science Framework (osf.io/fgm4s/)

\section{Notes}

Knowles 2018a

\begin{tabular}{ll}
\hline Trial name or title & Exploring the role of effort within the proximity effect \\
\hline Methods & Detailed in registration on Open Science Framework (osf.io/eqt92/)
\end{tabular}


Knowles 2018a (Continued)

Participants

\section{Interventions}

\section{Outcomes}

\section{Starting date}

Contact information

Daniel Knowles <Daniel.Knowles@bcu.ac.uk>

\section{Notes}

\section{Knowles 2018b}

Trial name or title

Exploring the role of visual salience within the proximity effect

\section{Methods}

Detailed in registration on Open Science Framework (osf.io/5gx9y/)

\section{Participants}

Interventions

Outcomes

Starting date

Contact information

Daniel Knowles <Daniel.Knowles@bcu.ac.uk>

\section{Notes}

Knowles 2018c

\begin{tabular}{ll} 
Trial name or title & Does the proximity effect occur through non-conscious processes? \\
\hline Methods & Detailed in registration on Open Science Framework (osf.io/zn256/)
\end{tabular}

\section{Participants}

Interventions

\section{Outcomes}

\section{Starting date}

\section{ADDITIONAL TABLES}




\begin{tabular}{|c|c|c|c|c|c|c|c|c|}
\hline Study & $\begin{array}{l}\text { Bias arising from } \\
\text { the randomisation } \\
\text { process }\end{array}$ & $\begin{array}{l}\text { Bias arising } \\
\text { from the tim- } \\
\text { ing of identi- } \\
\text { fication and } \\
\text { recruitment } \\
\text { of individual } \\
\text { participants } \\
\text { in relation to } \\
\text { timing of ran- } \\
\text { domisation } \\
\text { (CRCT only) }\end{array}$ & $\begin{array}{l}\text { Bias due to de- } \\
\text { viations from } \\
\text { intended inter- } \\
\text { ventions }\end{array}$ & $\begin{array}{l}\text { Bias due to } \\
\text { missing out- } \\
\text { come data }\end{array}$ & $\begin{array}{l}\text { Bias in mea- } \\
\text { surement of } \\
\text { the outcome }\end{array}$ & $\begin{array}{l}\text { Bias in selec- } \\
\text { tion of the re- } \\
\text { ported result }\end{array}$ & $\begin{array}{l}\text { Overall risk } \\
\text { of bias (se- } \\
\text { lection) }\end{array}$ & $\begin{array}{l}\text { Overall risk of } \\
\text { bias (consump- } \\
\text { tion) }\end{array}$ \\
\hline \multicolumn{9}{|l|}{ Availability studies } \\
\hline Fiske 2004 & Some concerns & Low risk & Low risk & Low risk & Low risk & Low risk & $\begin{array}{l}\text { Some con- } \\
\text { cerns }\end{array}$ & $N / A$ \\
\hline Foster 2014 & Low risk & Low risk & Low risk & Low risk & Low risk & Low risk & Low risk & $\mathrm{N} / \mathrm{A}$ \\
\hline Kocken 2012 & Some concerns & Low risk & Low risk & Low risk & Low risk & Low risk & $\begin{array}{l}\text { Some con- } \\
\text { cerns }\end{array}$ & $\mathrm{N} / \mathrm{A}$ \\
\hline Pechey 2019 & Some concerns & $\mathrm{N} / \mathrm{A}$ & Low risk & Low risk & Low risk & Low risk & $\begin{array}{l}\text { Some con- } \\
\text { cerns }\end{array}$ & $\mathrm{N} / \mathrm{A}$ \\
\hline Roe 2013 & Some concerns & $\mathrm{N} / \mathrm{A}$ & Low risk & Low risk & Low risk & Low risk & $\begin{array}{l}\text { Some con- } \\
\text { cerns }\end{array}$ & Some concerns \\
\hline Stubbs 2001 & Some concerns & $\mathrm{N} / \mathrm{A}$ & Low risk & Low risk & Low risk & Low risk & $\mathrm{N} / \mathrm{A}$ & Some concerns \\
\hline \multicolumn{9}{|l|}{ Proximity studies } \\
\hline Cohen 2015 & Some concerns & Low risk & Low risk & Low risk & Low risk & Low risk & $\begin{array}{l}\text { Some con- } \\
\text { cerns }\end{array}$ & Some concerns \\
\hline Engell 1996 (S1) & Some concerns & $\mathrm{N} / \mathrm{A}$ & Low risk & Low risk & Low risk & Low risk & $\mathrm{N} / \mathrm{A}$ & Some concerns \\
\hline Engell 1996 (S2) & Some concerns & $\mathrm{N} / \mathrm{A}$ & Low risk & Low risk & Low risk & Low risk & $\mathrm{N} / \mathrm{A}$ & Some concerns \\
\hline Greene 2017 & Some concerns & Low risk & Low risk & Low risk & Low risk & Low risk & $\begin{array}{l}\text { Some con- } \\
\text { cerns }\end{array}$ & Some concerns \\
\hline Hunter 2018 (S1) & Low risk & $\mathrm{N} / \mathrm{A}$ & Low risk & Low risk & Low risk & Low risk & $\mathrm{N} / \mathrm{A}$ & Low risk \\
\hline
\end{tabular}




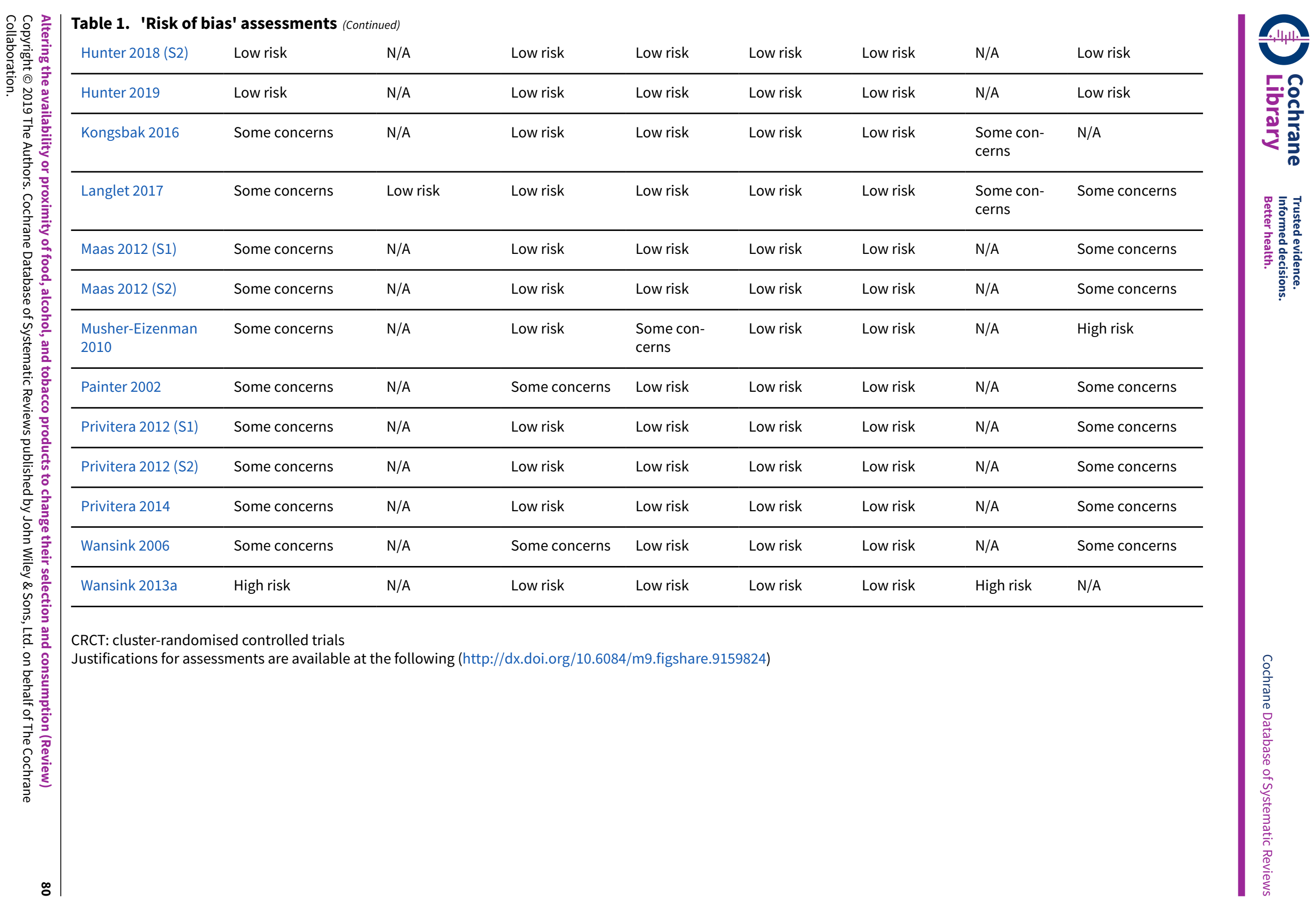




\section{AP PEN DICES}

\section{Appendix 1. Search strategies, search dates and yields}

\section{MEDLINE (OvidSP - including MEDLINE In-Process), 1946 to February week 32016}

Original search executed: 1st March 2016, records retrieved $=75026$

Updated search executed: 23 rd July 2018, records retrieved $=14185$

1. *Beverages/

2. *Alcohol Drinking/

3. (drink\$ or drunk\$ or alcohol\$ or beverage $\$ 1$ or beer $\$ 1$ or lager $\$ 1$ or wine $\$ 1$ or cider $\$ 1$ or alcopop\$1 or alco-pop $\$ 1$ or spirit or spirits or liquor $\$ 1$ or liquer $\$ 1$ or liqueur $\$ 1$ or whisky or whiskey or whiskies or whiskeys or schnapps or brandy or brandies or gin or gins or rum or rums or tequila 1 or vodka\$1 or cocktail\$1).ti,ab.

4. * $\mathrm{Tobacco} /$

5. *Smoking/

6. (cigar\$ or smok\$ or tobacco\$ or e-cig\$).ti,ab.

7. * Diet/

8. *Food/

9. *Food Intake/

10. *Food Habits/

11. *Food Preferences/

12. *Eating/

13. *Drinking/

14. *Food Dispensers, Automatic/

15. (nutri\$ or calori\$ or food\$ or eat or eats or eaten or eating or ate or low-fat or meal\$ or dessert $\$ 1$ or snack\$ or drink\$ or beverage $\$ 1) . t i, a b$.

16. ((increase $\$ 1$ or increasing or add $\$ 1$ or added or adding or addition $\$ 1$ or additional or introduce $\$ 1$ or introduction $\$ 1$ or introducing or extend\$ or reduc $\$$ or decrease $\$ 1$ or decreasing or remov\$ or restrict $\$$ or limit $\$$ or proxim $\$$ or distal or distanc $\$$ or position $\$$ or reposition $\$$ or visib\$ or accessib\$ or close or closer or closest or near or nearer or nearest or adjacent or far or farther or farthest or farthermost or further or furthest or furthermost) adj3 (amount $\$ 1$ or volume $\$ 1$ or quantity or quantities or availab\$ or range $\$$ or assortment $\$ 1$ or arrangement $\$ 1$ or array $\$$ or display $\$$ or choice $\$ 1$ or option $\$$ or item $\$ 1$ or effort or convenien $\$$ or salien $\$$ or product $\$ 1$ or packag\$ or portion\$ or serving $\$$ or glass or glasses or bottle\$ or dish $\$ 2$ or bowl\$1 or plate $\$ 1$ or box or boxes or boxed or bag or bags or bagged or packet\$ or carton\$1 or vending\$)).ti,ab.

17. ((increase $\$ 1$ or increasing or add\$1 or added or adding or addition\$1 or additional or introduce $\$ 1$ or introduction $\$ 1$ or introducing or extend\$ or reduc\$ or decrease $\$ 1$ or decreasing or remov\$ or restrict $\$$ or limit $\$$ or proxim $\$$ or distal or distanc\$ or position\$ or reposition \$ or visib\$ or accessib\$ or close or closer or closest or near or nearer or nearest or adjacent or far or farther or farthest or farthermost or further or furthest or furthermost) adj3 (food\$ or fruit $\$$ or vegetable $\$$ or FV or FFV or F\&V or low-fat or meal\$ or dessert $\$ 1$ or snack\$ or drink $\$$ or beverage 1 or alcohol\$ or cigar\$ or tobacco or e-cig\$)).ti,ab.

18. or $11-3$

19. or/4-6

20. or/7-15

21. 16 and 18

22. 16 and 19

Altering the availability or proximity of food, alcohol, and tobacco products to change their selection and consumption (Review) 
23. 16 and 20

24. or/17,21-23

25. exp animals/ not humans/

26. (rat or rats or mouse or mice or murine or rodent or rodents or hamster or hamsters or pig or pigs or porcine or rabbit or rabbits or animal or animals or dog or dogs or cat or cats or cow or cows or bovine or sheep or ovine or monkey or monkeys).ti,ab.

27. or/25-26

28. $24 \operatorname{not} 27$

29. (editorial or case reports or in vitro).pt.

30. 28 not 29

Embase (OvidSP), 1980 to Week 92016

Original search executed: 1 st March 2016, records retrieved $=68184$

Updated search executed: 23 rd July 2018, records retrieved $=4688$

1. *Beverage/

2. *Alcohol Consumption/

3. (drink\$ or drunk\$ or alcohol\$ or beverage $\$ 1$ or beer $\$ 1$ or lager $\$ 1$ or wine $\$ 1$ or cider $\$ 1$ or alcopop $\$ 1$ or alco-pop $\$ 1$ or spirit or spirits or liquor $\$ 1$ or liquer $\$ 1$ or liqueur $\$ 1$ or whisky or whiskey or whiskies or whiskeys or schnapps or brandy or brandies or gin or gins or rum or rums or tequila\$1 or vodka\$1 or cocktail\$1).ti,ab.

4. ${ }^{\star}$ Tobacco/

5. *Smoking/

6. (cigar\$ or smok\$ or tobacco\$ or e-cig\$).ti,ab.

7. *Diet/

8. *Food/

9. *Food Intake/

10. *Food Habits/

11. *Food Preferences/

12. *Eating/

13. *Drinking/

14. *Food Dispensers, Automatic/

15. (nutri\$ or calori or food\$ or eat or eats or eaten or eating or ate or low-fat or meal\$ or dessert $\$ 1$ or snack\$ or drink\$ or beverage $\$ 1)$.ti,ab.

16. ((increase $\$ 1$ or increasing or add $\$ 1$ or added or adding or addition $\$ 1$ or additional or introduce $\$ 1$ or introduction $\$ 1$ or introducing or extend $\$$ or reduc $\$$ or decrease $\$ 1$ or decreasing or remov\$ or restrict $\$$ or limit $\$$ or proxim $\$$ or distal or distanc $\$$ or position $\$$ or reposition $\$$ or visib\$ or accessib\$ or close or closer or closest or near or nearer or nearest or adjacent or far or farther or farthest or farthermost or further or furthest or furthermost) adj3 (amount $\$ 1$ or volume $\$ 1$ or quantity or quantities or availab\$ or range $\$$ or assortment $\$ 1$ or arrangement $\$ 1$ or array $\$$ or display $\$$ or choice $\$ 1$ or option $\$$ or item $\$ 1$ or effort or convenien $\$$ or salien $\$$ or product $\$ 1$ or packag or portion $\$$ or serving $\$$ or glass or glasses or bottle or dish $\$ 2$ or bowl\$1 or plate $\$ 1$ or box or boxes or boxed or bag or bags or bagged or packet $\$$ or carton $\$ 1$ or vending\$)).ti,ab.

17. ((increase $\$ 1$ or increasing or add $\$ 1$ or added or adding or addition $\$ 1$ or additional or introduce $\$ 1$ or introduction $\$ 1$ or introducing or extend\$ or reduc\$ or decrease 1 or decreasing or remov\$ or restrict $\$$ or limit or proxim or distal or distanc $\$$ or position\$ or reposition $\$$ or visib\$ or accessib\$ or close or closer or closest or near or nearer or nearest or adjacent or far or farther or farthest or farthermost or 
further or furthest or furthermost) adj3 (food\$ or fruit $\$$ or vegetable $\$$ or FV or FFV or F\&V or low-fat or meal\$ or dessert 1 or snack\$ or drink $\$$ or beverage $\$ 1$ or alcohol $\$$ or cigar $\$$ or tobacco or e-cig\$)).ti,ab.

18. or/1-3

19. or/4-6

20. or/7-15

21. 16 and 18

22. 16 and 19

23. 16 and 20

24. or $/ 17,21-23$

25. exp animals/ not humans/

26. (rat or rats or mouse or mice or murine or rodent or rodents or hamster or hamsters or pig or pigs or porcine or rabbit or rabbits or animal or animals or dog or dogs or cat or cats or cow or cows or bovine or sheep or ovine or monkey or monkeys).ti,ab.

27. or/25-26

28. $24 \operatorname{not} 27$

29. (editorial or case reports or in vitro).pt.

30. 28 not 29

PsycINFO (OvidSP) 1806 to February Week 42016

Original search executed: 1 st March 2016, records retrieved $=23813$

Updated search executed (in EBSCOhost): 23rd July 2018, records retrieved $=8267$

1. *Alcoholic Beverage/

2. *Beverages (Nonalcoholic)/

3. *Alcohol Drinking Patterns/

4. (drink\$ or drunk\$ or alcohol\$ or beverage $\$ 1$ or beer $\$ 1$ or lager $\$ 1$ or wine $\$ 1$ or cider $\$ 1$ or alcopop $\$ 1$ or alco-pop $\$ 1$ or spirit or spirits or liquor $\$ 1$ or liquer $\$ 1$ or liqueur $\$ 1$ or whisky or whiskey or whiskies or whiskeys or schnapps or brandy or brandies or gin or gins or rum or rums or tequila\$1 or vodka\$1 or cocktail\$1).ti,ab.

5. *Tobacco Smoking/

6. (cigar\$ or smok\$ or tobacco $\$$ or e-cig\$).ti,ab.

7. * Diets/

8. *Food/

9. *Food Intake/

10. *Food Preferences/

11. *Eating Behavior/

12. *Drinking Behavior/

13. (nutri\$ or calori\$ or food\$ or eat or eats or eaten or eating or ate or low-fat or meal\$ or dessert $\$ 1$ or snack\$ or drink\$ or beverage $\$ 1) . t i, a b$.

14. ( (increase $\$ 1$ or increasing or add $\$ 1$ or added or adding or addition $\$ 1$ or additional or introduce $\$ 1$ or introduction $\$ 1$ or introducing or extend $\$$ or reduc $\$$ or decrease $\$ 1$ or decreasing or remov $\$$ or restrict $\$$ or limit $\$$ or proxim $\$$ or distal or distanc $\$$ or position $\$$ or reposition $\$$ or visib\$ or accessib\$ or close or closer or closest or near or nearer or nearest or adjacent or far or farther or farthest or farthermost or further or furthest or furthermost) adj3 (amount $\$ 1$ or volume $\$ 1$ or quantity or quantities or availab\$ or range $\$$ or assortment $\$ 1$ or arrangement 
$\$ 1$ or array\$ or display $\$$ or choice $\$ 1$ or option $\$$ or item $\$ 1$ or effort or convenien $\$$ or salien $\$$ or product $\$ 1$ or packag $\$$ or portion $\$$ or serving $\$$ or glass or glasses or bottle\$ or dish $\$ 2$ or bowl\$1 or plate 1 or box or boxes or boxed or bag or bags or bagged or packet\$ or carton\$1 or vending\$)).ti,ab.

15. ((increase $\$ 1$ or increasing or add\$1 or added or adding or addition $\$ 1$ or additional or introduce $\$ 1$ or introduction $\$ 1$ or introducing or extend\$ or reduc\$ or decrease\$1 or decreasing or remov\$ or restrict $\$$ or limit or proxim\$ or distal or distanc\$ or position\$ or reposition $\$$ or visib\$ or accessib\$ or close or closer or closest or near or nearer or nearest or adjacent or far or farther or farthest or farthermost or further or furthest or furthermost) adj3 (food\$ or fruit $\$$ or vegetable\$ or FV or FFV or F\&V or low-fat or meal\$ or dessert\$1 or snack\$ or drink $\$$ or beverage 1 or alcohol\$ or cigar\$ or tobacco or e-cig\$)).ti,ab.

16. or $/ 1-4$

17. or/5-6

18. or $/ 7-13$

19. 14 and 16

20. 14 and 17

21. 14 and 18

22. or $/ 15,19-21$

23. limit 22 to human

Cochrane Central Register of Controlled Trials (CENTRAL), 1992 to 3rd March 2016 (Issue 2 of 12, 2016)

Original search executed: 3 rd March 2016, records retrieved $=15300$

Updated search executed: 23 rd July 2018, records retrieved $=4620$

((drink ${ }^{\star}$ OR drunk ${ }^{\star}$ OR alcohol* OR beverage* OR beer* OR lager* OR wine* OR cider ${ }^{\star}$ OR alcopop* OR alco-pop* OR spirit OR spirits OR liquor* OR liquer* OR liqueur* OR whisky OR whiskey OR whiskies OR whiskeys OR schnapps OR brandy OR brandies OR gin OR gins OR rum OR rums OR tequila* OR vodka* OR cocktail ${ }^{\star}$ OR cigar ${ }^{\star}$ OR smok* OR tobacco* OR e-cig* OR nutri* OR calori ${ }^{\star}$ OR food ${ }^{\star}$ OR eat OR eats OR eaten OR eating OR ate OR low-fat OR meal ${ }^{\star}$ OR dessert* OR snack ${ }^{\star}$ ) AND ((increase* OR increasing OR add OR adds OR added OR adding OR addition OR additions OR additional OR introduce* OR introduction ${ }^{\star}$ OR introducing OR extend ${ }^{\star}$ OR reduc $^{\star}$ OR decrease $^{\star}$ OR decreasing OR remov* OR restrict* OR limit* OR proxim* OR distal OR distanc* OR position* OR reposition* OR visib* OR accessib* OR close OR closer OR closest OR nearer OR nearest OR adjacent OR far OR farther OR farthest OR farthermost OR further OR furthest OR furthermost) NEAR/6 (amount OR amounts OR volume* OR quantity OR quantities OR availab* OR range* OR assortment* OR arrangement* OR array* OR display ${ }^{\star}$ OR choice* OR option* OR item OR items OR effort* OR convenien* OR salien* OR product OR products OR package* OR portion* OR serving* OR glass OR glasses OR bottle* OR dish OR dishes OR bowl OR bowls OR plate OR plates OR plated OR box OR boxes OR boxed OR bag OR bags OR bagged OR packet ${ }^{\star}$ OR carton ${ }^{\star}$ OR vending $\left.{ }^{\star}\right)$ ))

OR

((increase* OR increasing OR add OR adds OR added OR adding OR addition OR additions OR additional OR introduce* OR introduction* OR introducing OR extend ${ }^{\star}$ OR reduc* OR decrease ${ }^{\star}$ OR decreasing OR remov* OR restrict ${ }^{\star}$ OR limit ${ }^{\star}$ OR proxim* OR distal OR distanc ${ }^{\star}$ OR position* OR reposition* OR visib* OR accessib* OR close OR closer OR closest OR nearer OR nearest OR adjacent OR far OR farther OR farthest OR farthermost OR further OR furthest OR furthermost) NEAR/6 (food* OR fruit* OR vegetable* OR FV OR FFV OR F\&V OR low-fat OR meal ${ }^{\star}$ OR dessert* OR snack* OR drink* OR beverage* OR alcohol* OR cigar ${ }^{\star}$ OR tobacco OR e-cig $\left.{ }^{\star}\right)$ )

NOT

(rat or rats or mouse or mice or murine or rodent or rodents or hamster or hamsters or pig or pigs or porcine or rabbit or rabbits or animal or animals or dog or dogs or cat or cats or cow or cows or bovine or sheep or ovine or monkey or monkeys)

\section{Applied Social Sciences Index and Abstracts (ProQuest) 1987 to 2nd March 2016}

Original search executed: 2 nd March 2016, records retrieved $=762$

Updated search executed: 24 th July 2018, records retrieved $=4398$

((drink ${ }^{\star}$ OR drunk ${ }^{\star}$ OR alcohol ${ }^{\star}$ OR beverage $\left[{ }^{\star} 1\right]$ OR beer $\left[{ }^{\star} 1\right]$ OR lager $\left[{ }^{\star} 1\right]$ OR wine $\left[{ }^{\star} 1\right]$ OR cider $\left[{ }^{\star} 1\right]$ OR alcopop $\left[{ }^{\star} 1\right]$ OR alco-pop $\left[{ }^{\star} 1\right]$ OR spirit OR spirits OR liquor[ $\left.{ }^{\star} 1\right]$ OR liquer[ $\left.{ }^{\star} 1\right]$ OR liqueur [ $\left.{ }^{\star} 1\right]$ OR whisky OR whiskey OR whiskies OR whiskeys OR schnapps OR brandy OR brandies OR gin OR gins OR rum OR rums OR tequila[ $\left.{ }^{\star} 1\right]$ OR vodka[ $\left.{ }^{\star} 1\right]$ OR cocktail[ [*1] OR cigar ${ }^{\star}$ OR smok ${ }^{\star}$ OR tobacco* OR e-cig ${ }^{\star}$ OR nutri ${ }^{\star}$ OR calori ${ }^{\star}$ 
OR food ${ }^{\star}$ OR eat OR eats OR eaten OR eating OR ate OR low-fat OR meal ${ }^{\star}$ OR dessert[ $\left.{ }^{\star} 1\right]$ OR snack $\left.{ }^{\star}\right)$ AND ((increase[ $\left.{ }^{\star} 1\right]$ OR increasing OR add [ $\left.{ }^{\star} 1\right]$ OR added OR adding OR addition [ $\left.{ }^{*} 1\right]$ OR additional OR introduce[ $\left.{ }^{\star} 1\right]$ OR introduction[ $\left.{ }^{\star} 1\right]$ OR introducing OR extend ${ }^{\star}$ OR reduc ${ }^{\star}$ OR decrease[*1] OR decreasing OR remov* OR restrict* OR limit* OR proxim* OR distal OR distanc* OR position* OR reposition* OR visib* OR accessib* OR close OR closer OR closest OR nearer OR nearest OR adjacent OR far OR farther OR farthest OR farthermost OR further OR furthest OR furthermost) NEAR/6 (amount[^1] OR volume[*1] OR quantity OR quantities OR availab* OR range* OR assortment[*1] OR

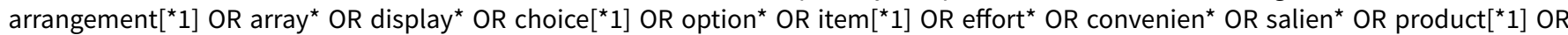
package ${ }^{\star}$ OR portion* OR serving* OR glass OR glasses OR bottle ${ }^{\star}$ OR dish OR dishes OR bowl[ $\left.{ }^{\star} 1\right]$ OR plate[ $\left.{ }^{\star} 1\right]$ OR box OR boxes OR boxed OR bag OR bags OR bagged OR packet* OR carton[ $\left.{ }^{\star} 1\right]$ OR vending $\left.\left.{ }^{\star}\right)\right)$ ) OR ((increase[ $\left.{ }^{\star} 1\right]$ OR increasing OR add[ [ 1$]$ OR added OR adding OR addition [ $\left.{ }^{\star} 1\right]$ OR additional OR introduce[ $\left.{ }^{\star} 1\right]$ OR introduction [ $\left.{ }^{\star} 1\right]$ OR introducing OR extend ${ }^{\star}$ OR reduc ${ }^{\star}$ OR decrease[ $\left.{ }^{\star} 1\right]$ OR decreasing OR remov $^{\star}$ OR restrict* OR limit* OR proxim* OR distal OR distanc* OR position* OR reposition* OR visib* OR accessib* OR close OR closer OR closest OR nearer OR nearest OR adjacent OR far OR farther OR farthest OR farthermost OR further OR furthest OR furthermost) NEAR/6 (food ${ }^{\star}$ OR fruit* OR vegetable* OR FV OR FFV OR low-fat OR meal ${ }^{\star}$ OR dessert[ [ 1 ] OR snack ${ }^{\star}$ OR drink ${ }^{\star}$ OR beverage [ $\left.{ }^{\star} 1\right]$ OR alcohol ${ }^{\star}$ OR cigar $^{\star}$ OR tobacco OR e-cig*)) NOT (rat or rats or mouse or mice or murine or rodent or rodents or hamster or hamsters or pig or pigs or porcine or rabbit or rabbits or animal or animals or dog or dogs or cat or cats or cow or cows or bovine or sheep or ovine or monkey or monkeys)

Science Citation Index Expanded (Web of Science) (1900 to 2nd March 2016) + Social Sciences Citation Index (Web of Science) (1956 to 2nd March 2016) + Conference Proceedings Citation Index - Science (Web of Science) (1990 to 2nd March 2016) + Conference Proceedings Citation Index - Social Science \& Humanities (Web of Science) (1990 to 2nd March 2016)

Original search executed: 2 nd March 2016, records retrieved $=50164$

Updated search executed: 24 th July 2018, records retrieved $=8922$

$\# 1$

TS $=\left(\right.$ drink $^{\star}$ OR drunk ${ }^{\star}$ OR alcohol ${ }^{\star}$ OR beverage $\left[{ }^{\star} 1\right]$ OR beer $\left[{ }^{\star} 1\right]$ OR lager[ $\left[{ }^{\star} 1\right]$ OR wine $\left[{ }^{\star} 1\right]$ OR cider $\left[{ }^{\star} 1\right]$ OR alcopop $\left[{ }^{\star} 1\right]$ OR alco-pop $\left[{ }^{\star} 1\right]$ OR spirit OR spirits OR liquor $\left[{ }^{\star} 1\right]$ OR liquer[ $\left.{ }^{\star} 1\right]$ OR liqueur $\left[{ }^{\star} 1\right]$ OR whisky OR whiskey OR whiskies OR whiskeys OR schnapps OR brandy OR

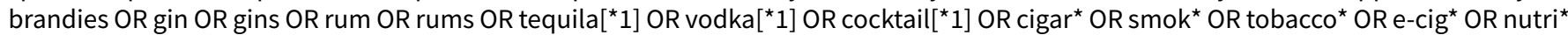
OR calori ${ }^{\star}$ OR food ${ }^{\star}$ OR eat OR eats OR eaten OR eating OR ate OR low-fat OR meal ${ }^{\star}$ OR dessert [ $\left.{ }^{\star} 1\right]$ OR snack* OR fruit $^{\star}$ OR vegetable* OR FV OR FFV OR F\&V OR item [ $\left.{ }^{\star} 1\right]$ OR product[ $\left.{ }^{\star} 1\right]$ OR package ${ }^{\star}$ OR portion* OR serving ${ }^{\star}$ OR glass OR glasses OR bottle* OR dish OR dishes OR bowl[^1] OR plate[ $\left.{ }^{\star} 1\right]$ OR box OR boxes OR boxed OR bag OR bags OR bagged OR packet ${ }^{\star}$ OR carton[ $\left.{ }^{\star} 1\right]$ OR vending $\left.{ }^{\star}\right)$

Indexes=SCI-EXPANDED, SSCI, CPCI-S, CPCI-SSH Timespan=All years

\# 2

TS=(proxim* OR distal OR distanc ${ }^{\star}$ OR position* OR reposition* OR visib* OR accessib* OR availab* OR range* OR assortment[ $\left.{ }^{\star} 1\right]$ OR arrangement [ $\left.{ }^{\star} 1\right]$ OR array ${ }^{\star}$ OR display ${ }^{\star}$ OR choice[ $\left.{ }^{\star} 1\right]$ OR option ${ }^{\star}$ OR effort OR convenien ${ }^{\star}$ OR salien ${ }^{\star}$ )

Indexes=SCI-EXPANDED, SSCI, CPCI-S, CPCI-SSH Timespan=All years

\# 3

\#2 AND \#1

Indexes=SCI-EXPANDED, SSCI, CPCI-S, CPCI-SSH Timespan=All years

\# 4

$\mathrm{TS}=$ (rat or rats or mouse or mice or murine or rodent or rodents or hamster or hamsters or pig or pigs or porcine or rabbit or rabbits or animal or animals or dog or dogs or cat or cats or cow or cows or bovine or sheep or ovine or monkey or monkeys)

Indexes=SCI-EXPANDED, SSCI, CPCI-S, CPCI-SSH Timespan=All years

\#5

\#3 NOT \#4

Indexes=SCI-EXPANDED, SSCI, CPCI-S, CPCI-SSH Timespan=All years

\#6

\#3 NOT \#4

Refined by: [excluding] WEB OF SCIENCE CATEGORIES: ( MATERIALS SCIENCE MULTIDISCIPLINARY OR WATER RESOURCES OR MECHANICS OR ENGINEERING ELECTRICAL ELECTRONIC OR OCEANOGRAPHY OR CELL BIOLOGY OR ENVIRONMENTAL SCIENCES OR COMPUTER 
SCIENCE INFORMATION SYSTEMS OR ELECTROCHEMISTRY OR FOOD SCIENCE TECHNOLOGY OR ENERGY FUELS OR NUCLEAR SCIENCE TECHNOLOGY OR CHEMISTRY PHYSICAL OR AGRICULTURE MULTIDISCIPLINARY OR FORESTRY OR PLANT SCIENCES OR MATERIALS SCIENCE CERAMICS OR BIOPHYSICS OR ECOLOGY OR ZOOLOGY OR CLINICAL NEUROLOGY OR PHYSICS APPLIED OR SOIL SCIENCE OR BIOCHEMISTRY MOLECULAR BIOLOGY OR ENGINEERING MECHANICAL OR BIOLOGY OR CHEMISTRY ANALYTICAL OR GENETICS HEREDITY OR CHEMISTRY INORGANIC NUCLEAR OR COMPUTER SCIENCE THEORY METHODS OR COMPUTER SCIENCE SOFTWARE ENGINEERING OR CHEMISTRY MULTIDISCIPLINARY OR THERMODYNAMICS OR ENGINEERING BIOMEDICAL OR OPTICS OR PHYSICS ATOMIC MOLECULAR CHEMICAL OR MATERIALS SCIENCE COATINGS FILMS OR ENGINEERING MANUFACTURING OR MARINE FRESHWATER BIOLOGY OR BIODIVERSITY CONSERVATION OR BIOTECHNOLOGY APPLIED MICROBIOLOGY OR COMPUTER SCIENCE INTERDISCIPLINARY APPLICATIONS OR CARDIAC CARDIOVASCULAR SYSTEMS OR POLYMER SCIENCE OR PHYSICS MULTIDISCIPLINARY OR DENTISTRY ORAL SURGERY MEDICINE OR PHYSICS CONDENSED MATTER OR ONCOLOGY OR IMMUNOLOGY OR AGRONOMY OR METALLURGY METALLURGICAL ENGINEERING OR EVOLUTIONARY BIOLOGY OR ENGINEERING CHEMICAL OR FISHERIES OR IMAGING SCIENCE PHOTOGRAPHIC TECHNOLOGY OR TELECOMMUNICATIONS OR TOXICOLOGY OR CHEMISTRY APPLIED OR ENTOMOLOGY OR RESPIRATORY SYSTEM OR PHARMACOLOGY PHARMACY OR COMPUTER SCIENCE ARTIFICIAL INTELLIGENCE OR AGRICULTURE DAIRY ANIMAL SCIENCE OR HORTICULTURE OR ENDOCRINOLOGY METABOLISM OR ENGINEERING MULTIDISCIPLINARY OR GEOSCIENCES MULTIDISCIPLINARY OR GEOCHEMISTRY GEOPHYSICS OR CHEMISTRY ORGANIC OR ASTRONOMY ASTROPHYSICS OR AGRICULTURAL ENGINEERING OR AUTOMATION CONTROL SYSTEMS OR INSTRUMENTS INSTRUMENTATION OR RADIOLOGY NUCLEAR MEDICINE MEDICAL IMAGING OR PHYSICS PARTICLES FIELDS OR COMPUTER SCIENCE HARDWARE ARCHITECTURE OR CHEMISTRY MEDICINAL OR NANOSCIENCE NANOTECHNOLOGY OR SPECTROSCOPY OR MICROBIOLOGY OR ENGINEERING CIVIL OR ENGINEERING ENVIRONMENTAL OR METEOROLOGY ATMOSPHERIC SCIENCES OR PERIPHERAL VASCULAR DISEASE OR SURGERY OR GASTROENTEROLOGY HEPATOLOGY OR LIMNOLOGY OR BIOCHEMICAL RESEARCH METHODS OR GERIATRICS GERONTOLOGY OR SOCIAL WORK OR REHABILITATION OR ENGINEERING INDUSTRIAL OR MATERIALS SCIENCE COMPOSITES OR ANESTHESIOLOGY OR VIROLOGY OR FAMILY STUDIES OR STATISTICS PROBABILITY OR AUDIOLOGY SPEECH LANGUAGE PATHOLOGY OR PEDIATRICS OR MATHEMATICS APPLIED OR COMMUNICATION OR SOCIOLOGY OR POLITICAL SCIENCE OR ORNITHOLOGY OR ORTHOPEDICS OR AGRICULTURAL ECONOMICS POLICY OR DEVELOPMENTAL BIOLOGY OR SPORT SCIENCES OR CRITICAL CARE MEDICINE OR EDUCATION SCIENTIFIC DISCIPLINES OR ACOUSTICS OR PARASITOLOGY OR MEDICAL INFORMATICS OR UROLOGY NEPHROLOGY OR ENGINEERING AEROSPACE OR INFORMATION SCIENCE LIBRARY SCIENCE OR OBSTETRICS GYNECOLOGY OR OPERATIONS RESEARCH MANAGEMENT SCIENCE OR LAW OR PATHOLOGY OR ANATOMY MORPHOLOGY OR MEDICINE LEGAL OR PRIMARY HEALTH CARE OR EDUCATION EDUCATIONAL RESEARCH OR PLANNING DEVELOPMENT OR MATHEMATICS OR MEDICAL LABORATORY TECHNOLOGY OR MATERIALS SCIENCE PAPER WOOD OR DERMATOLOGY OR PHYSICS NUCLEAR OR PHYSICS FLUIDS PLASMAS OR ANTHROPOLOGY OR WOMEN S STUDIES OR MANAGEMENT OR GEOGRAPHY OR MATHEMATICAL COMPUTATIONAL BIOLOGY OR OPHTHALMOLOGY OR MATHEMATICS INTERDISCIPLINARY APPLICATIONS OR PHYSICS MATHEMATICAL OR TROPICAL MEDICINE OR SOCIAL SCIENCES MATHEMATICAL METHODS OR NURSING OR HEMATOLOGY OR SOCIAL ISSUES OR BUSINESS OR CRYSTALLOGRAPHY OR BUSINESS FINANCE OR VETERINARY SCIENCES OR REPRODUCTIVE BIOLOGY OR PSYCHOLOGY DEVELOPMENTAL OR TRANSPORTATION OR MINERALOGY OR PALEONTOLOGY OR OTORHINOLARYNGOLOGY OR GERONTOLOGY OR INFECTIOUS DISEASES OR EMERGENCY MEDICINE OR LINGUISTICS OR MYCOLOGY OR CRIMINOLOGY PENOLOGY OR RHEUMATOLOGY OR INTERNATIONAL RELATIONS OR GEOLOGY OR PUBLIC ADMINISTRATION OR GEOGRAPHY PHYSICAL OR ROBOTICS OR ARCHITECTURE OR DEMOGRAPHY OR ANDROLOGY OR HISTORY PHILOSOPHY OF SCIENCE OR HISTORY OR AREA STUDIES OR HEALTH CARE SCIENCES SERVICES OR URBAN STUDIES OR HEALTH POLICY SERVICES OR PSYCHOLOGY EDUCATIONAL OR ALLERGY OR PSYCHOLOGY MATHEMATICAL OR MINING MINERAL PROCESSING OR TRANSPORTATION SCIENCE TECHNOLOGY OR COMPUTER SCIENCE CYBERNETICS OR CONSTRUCTION BUILDING TECHNOLOGY OR ETHICS OR INDUSTRIAL RELATIONS LABOR OR ENGINEERING GEOLOGICAL OR MEDICAL ETHICS OR PSYCHOLOGY PSYCHOANALYSIS OR MATERIALS SCIENCE TEXTILES OR ETHNIC STUDIES OR REMOTE SENSING OR ENGINEERING PETROLEUM OR INTEGRATIVE COMPLEMENTARY MEDICINE OR HISTORY OF SOCIAL SCIENCES OR MATERIALS SCIENCE CHARACTERIZATION TESTING OR PHILOSOPHY OR MATERIALS SCIENCE BIOMATERIALS OR ENGINEERING MARINE OR MICROSCOPY OR HUMANITIES MULTIDISCIPLINARY OR PSYCHIATRY OR NEUROIMAGING OR LITERARY THEORY CRITICISM OR LITERATURE OR ART OR LITERATURE GERMAN DUTCH SCANDINAVIAN OR RELIGION OR POETRY OR EDUCATION SPECIAL OR MEDIEVAL RENAISSANCE STUDIES OR CELL TISSUE ENGINEERING OR LITERATURE AMERICAN OR FILM RADIO TELEVISION OR CULTURAL STUDIES OR CLASSICS OR ENGINEERING OCEAN OR ASIAN STUDIES OR LANGUAGE LINGUISTICS OR THEATER OR ARCHAEOLOGY OR LITERATURE SLAVIC OR MUSIC OR LITERATURE ROMANCE OR TRANSPLANTATION OR DANCE)

Indexes=SCI-EXPANDED, SSCI, CPCI-S, CPCI-SSH Timespan=All years

These exclusions mean that the search is focused on the following:

Searching in PUBLIC ENVIRONMENTAL OCCUPATIONAL HEALTH $(12,220)$ MEDICINE RESEARCH EXPERIMENTAL $(1,224)$ SOCIAL SCIENCES INTERDISCIPLINARY (606) NUTRITION DIETETICS (10,318) PSYCHOLOGY (1,163) PSYCHOLOGY SOCIAL (546) MULTIDISCIPLINARY SCIENCES $(9,815)$ BEHAVIORAL SCIENCES $(1,132)$ PSYCHOLOGY BIOLOGICAL $(410)$ MEDICINE GENERAL INTERNAL $(7,521)$ PSYCHOLOGY EXPERIMENTAL (791) ENVIRONMENTAL STUDIES (310) SUBSTANCE ABUSE (5,107) ECONOMICS (774) HOSPITALITY LEISURE SPORT TOURISM (162) PSYCHOLOGY CLINICAL (2,392) SOCIAL SCIENCES BIOMEDICAL (663) PSYCHOLOGY APPLIED (117) PSYCHOLOGY MULTIDISCIPLINARY (1,389) PHYSIOLOGY (658) ERGONOMICS (38)

Trials Register of Promoting Health Interventions (EPPI Centre) (2004 to 4th March 2016)

Original search executed: 4 th March 2016, records retrieved $=747$

Updated search executed: 27 th July 2018, records retrieved $=19$

Altering the availability or proximity of food, alcohol, and tobacco products to change their selection and consumption (Review) 
110 Focus of the report: alcohol OR healthy eating OR tobacco

111 Type(s) of intervention: environmental modification

\section{AND 111}

113 Freetext (item record) "proximity"

114 Freetext (item record) "proximal”

115 Freetext (item record) "distal”

116 Freetext (item record) "distanc"

117 Freetext (item record) "position*"

118 Freetext (item record) "reposition"”

119 Freetext (item record) "visib*"

120 Freetext (item record) "accessib"

121 Freetext (item record) "effort"”

122 Freetext (item record) "convenien "

123 Freetext (item record) "salien*”

124 Freetext (item record) "availab"”

125 Freetext (item record) "range

126 Freetext (item record) "assortment*"

127 Freetext (item record) "arrangement ${ }^{\star \prime}$

128 Freetext (item record) "array "

129 Freetext (item record) "display "

130 Freetext (item record) "choice ${ }^{\star}$ "

131 Freetext (item record) "option"”

132113 OR 114 OR 115 OR 116 OR 117 OR 118 OR 119 OR 120 OR 121 OR 122 OR 123 OR 124 OR 125 OR 126 OR 127

OR 128 OR 129 OR 130 OR 131

133 Freetext (item record) "drink *”

134 Freetext (item record) "drunk "”

135 Freetext (item record) "alcohol *"

136 Freetext (item record) "beverage "

137 Freetext (item record) "beer"

138 Freetext (item record) "lager*"

139 Freetext (item record) "wine

140 Freetext (item record) "cider"”

141 Freetext (item record) "alcopop"

142 Freetext (item record) "alco-pop*”

143 Freetext (item record) "spirit" 
144 Freetext (item record) "spirits"

145 Freetext (item record) "liquor"

146 Freetext (item record) "liquer"

147 Freetext (item record) "liqueur"

148 Freetext (item record) "whisk "”

149 Freetext (item record) "schnapps"

150 Freetext (item record) "brandy"

151 Freetext (item record) "brandies"

152 Freetext (item record) "gin"

153 Freetext (item record) "gins"

154 Freetext (item record) "rum"

155 Freetext (item record) "rums"

156 Freetext (item record) "tequila*"

157 Freetext (item record) "vodka*”

158 Freetext (item record) "cocktail ${ }^{\star ”}$

159 Freetext (item record) "cigar"

160 Freetext (item record) "smoke"

161 Freetext (item record) "smokes"

162 Freetext (item record) "smoking"

163 Freetext (item record) "smoker"

164 Freetext (item record) "smokers"

165 Freetext (item record) "smoked"

166 Freetext (item record) "tobacco*”

167 Freetext (item record) "nutri ${ }^{\star}$ ”

168 Freetext (item record) “calori ${ }^{\star ”}$

169 Freetext (item record) "food"”

170 Freetext (item record) "eat"

171 Freetext (item record) "eats"

172 Freetext (item record) "eaten"

173 Freetext (item record) "eating"

174 Freetext (item record) "ate"

175 Freetext (item record) "low-fat"

176 Freetext (item record) "meal *"

177 Freetext (item record) "dessert*"

178 Freetext (item record) "snack *”

Altering the availability or proximity of food, alcohol, and tobacco products to change their selection and consumption (Review) 


\author{
179 Freetext (item record) "fruit" \\ 180 Freetext (item record) "vegetable*” \\ 181 Freetext (item record) "FV" \\ 182 Freetext (item record) "FFV" \\ 183 Freetext (item record) "F\&V"
}

184133 OR 134 OR 135 OR 136 OR 137 OR 138 OR 139 OR 140 OR 141 OR 142 OR 143 OR 144 OR 145 OR 146 OR 147 OR 148 OR 149 OR 150 OR 151 OR 152 OR 153 OR 154 OR 155 OR 156 OR 157 OR 158 OR 159 OR 160 OR 161 OR 162 OR 163 OR 164 OR 165 OR 166 OR 167 OR 168 OR 169 OR 170 OR 171 OR 172 OR 173 OR 174 OR 175 OR 176 OR 177 OR 178 OR 179 OR 180 OR 181 OR 182 OR 183

185132 AND 184

186112 OR 185

\title{
Appendix 2. Details of the semi-automated screening workflow
}

The semi-automated screening workflow proceeded in four phases: i) Initial sample; ii) Active learning; iii) Topic modelling; iv) Active learning (final phase). A separate methods paper is being developed that will describe these processes in greater detail.

\section{Initial sample}

Firstly, we screened a random sample of title-abstract records to establish an initial estimate of the baseline inclusion rate (Shemilt 2014), to both inform prospective monitoring of the performance of the semi-automated screening workflow and to supply an unbiased initial sample of records for machine learning (see 'Active learning', below).

\section{Active learning}

Secondly, we deployed active learning with the aim of identifying records of potentially eligible studies as rapidly as possible. In this phase, title-abstract records were prioritised for manual screening using active learning, whereby the machine iteratively 'learns' to distinguish between relevant and irrelevant records in conjunction with manual user input (Miwa 2014). We previously deployed this method in two large-scale systematic scoping reviews of interventions to change health behaviour (Shemilt 2013; Hollands 2013a; Shemilt 2014). Active learning was initially trained using small samples of provisionally included and excluded records drawn from a reference set of 24 records of potentially eligible studies identified by a published scoping review on physical micro-environment interventions (Hollands $2013 \mathrm{a}$ ), and in the random sample of citations screened in the first phase (Initial sample). In order to deploy active learning, a stopping criterion is needed that prespecifies when this phase will be truncated. We set the stopping criterion in terms of the maximum marginal resource the review team was willing to 'pay' in order to identify one additional title-abstract record of a potentially eligible study. We prospectively monitored and recorded screening time-on-task and stopped the active learning phase of the semi-automated workflow when the review authors had completed 15 hours of duplicate screening (i.e. 30 hours time-on-task in total for two review authors) without identifying any further records of potentially eligible studies. In practice, this equated to a figure of 1700 title-abstract records. At this point, we also screened a second random sample of records to establish a second estimate of the baseline inclusion rate (Shemilt 2014). In this active learning phase of the workflow, we alternated between title-abstract and full-text screening stages after each set of 2400 title-abstract records had been manually screened. This was intended to promote more accurate initial title-abstract screening decisions, and to enable retrospective modelling of the impact of using full-text screening decisions in training data for active learning.

\section{Topic modelling}

Active learning was expected to have identified the large majority of title-abstract records of potentially eligible studies that were present in the full set retrieved by electronic searches before the above stopping criterion for that phase was enacted. However, given that active learning iteratively prioritises further title-abstract records for screening based on the researchers' preceding eligibility decisions about records that were also prioritised by active learning (i.e. the algorithm progressively finds 'more of the same'), we introduced an entirely different, novel method into the semi-automated workflow, in order to provide a check and balance on the use of active learning alone. In this third phase of title-abstract screening, records were allocated for duplicate manual screening based on topic modelling using Latent Dirichlet Allocation (LDA) (Pedregosa 2011). Topic modelling essentially clusters title-abstract records according to the combinations of terms they contain and returns a set of 'topic terms' for each cluster (hereafter, a 'topic').

Topic modelling was used to generate 50 topics underlying the full set of title-abstracts retrieved by electronic searches (or included amongst the reference set), and concurrently to generate a series of 'membership scores' for each unscreened record, by topic. The membership score is based on the computed probability that a record is described by the topic (i.e. a higher membership score reflects a higher probability of membership of the topic) and is $>0$ for all records in all topics. Each unscreened title-abstract record was then allocated to the single topic that corresponded with its highest membership score. Results of a preliminary simulation study, conducted to simulate this phase of the workflow in a screening dataset curated from another Cochrane Review (Hollands 2015), indicated that the large majority of generated topics contained no unscreened records of potentially eligible studies (i.e. most topics are irrelevant), and also 
that the review authors were able to discriminate accurately between topics that contained the most and fewest records of potentially eligible studies when blinded to this information. Two review authors therefore examined each topic, blinded to the number of records allocated to each, and placed the 50 topics in rank order based on their inter-subjective judgement of the likelihood that each set of terms describes a set of records that includes eligible studies. A second ranking of the 50 topics was also generated based on the number of potentially eligible title-abstract records each topic contained amongst records already screened up to the end of the active learning phase (i.e. a data-generated ranking). We then computed a composite ranking by adding together the review authors' ranking and the datagenerated ranking, once the latter had been multiplied by 0.5 . This procedure assigns double weight to the review authors' judgements in the composite ranking, promoting those topics that the review authors rank higher but that contain a relatively low number of potentially eligible title-abstract records amongst those already screened (and, conversely, demoting those topics that the authors rank lower but that contain a relatively low number of potentially eligible title-abstract records amongst those already screened).

At the end of the active learning phase, the 'remaining screening budget' (i.e. the 'overall screening budget' minus the number of records already screened) was calculated and allocated between topics, by drawing a random sample of unscreened title-abstract records from each topic (i.e. the sum of the sizes of the 50 random samples equalled the remaining screening budget). The sizes of random samples drawn from topics were scaled to approximate a beta distribution $(\alpha=0.3, \beta=3.0)$ across rank-ordered topics (highest to lowest), in order to reflect our prior belief (informed by results of the simulation study) about the likely distribution of any further potentially eligible titleabstract records across rank-ordered topics. Sampled records were then allocated for duplicate manual screening in topic rank order, from highest to lowest ranked. This procedure ensured that records assigned to a higher-ranked topic were more likely to be allocated for screening, relative to those assigned to a lower-ranked topic. We continued the topic modelling phase of title-abstract screening until either all records allocated using the above procedure had been screened, or the early stopping criterion was enacted, which was the case. This stopping rule was based on prospective monitoring of time-on-task, and required that we truncated this phase of the semi-automated screening workflow when review authors had completed 15 hours of duplicate screening (i.e. 30 hours time-on-task in total for two review authors) without identifying any further records of potentially eligible studies. As previously, the stopping criterion was set at 1700 titleabstract records.

\section{Active learning (final phase)}

Because the topic modelling phase may detect additional title-abstract records that alter any subsequent prioritisation of records by active learning, we conducted a final phase of screening using the active learning method outlined above, truncated according to the same stopping criterion (15 hours of duplicate screening, that being 1700 records screened). Including this further phase gave additional confidence that, within available resources, all relevant title-abstract records had been identified.

\section{Use of semi-automated screening workflow for review updates}

Following the original searches conducted in March 2016, the searches were subsequently updated in July 2018. A simplified version of the workflow described above was then applied to the updated searches. This involved an active learning process that was able to learn from the coding decisions made in the initial screening process, with a model being constructed based on the large number of include and exclude decisions from the initial title-abstract screening (in which 27,116 records were screened). This model was then applied to the updated searches to rank title-abstract records by their likely relevance. The ranked records were then screened in descending order of highest-lowest likely relevance until the same stopping rule as was previously employed was met (i.e. 1700 title-abstract records without identifying a potentially eligible record, based on time-on-task estimates). A topic modelling process was not used for screening the updated searches, because in the initial screening all of the included studies were identified as a result of the active learning phase, and therefore topic modelling had provided no additional value.

\section{Appendix 3. Meta-regression analyses}

\section{Procedure}

Univariate meta-regression analyses were conducted on the following covariates for stages 2, 3, and 4.

Stage 2:

1. Study design (categorical)

2. Study setting (categorical)

3. Product healthiness (categorical)

4. Duration of exposure to invention (categorical)

5. Socioeconomic status (categorical)

6. Product-outcome relationship (categorical)

7. Timing of outcome measurement (categorical) 
8. Risk of bias summary (categorical)

Stage 3:

1. Absolute difference in proximity (continuous)

Stage 4:

1. Percentage of female participants (continuous)

We had intended that covariates within each stage would be combined as a multivariate analysis to identify the set of covariates that best explained the statistical heterogeneity observed during meta-analysis. A final meta-regression model would therefore be formulated that included covariates from all three stages.

The following procedure was to be used to select and incorporate covariates into the multivariate model.

1. Covariates identified as potentially important predictors of the outcome in stages 2,3 , and 4 ranked in order of $R^{2}$ values.

2. A stepwise procedure used to add a covariate to the top-ranked covariate to a multivariable meta-regression model.

3. A covariate is retained in the multivariable model should a significant effect be observed for the covariate, the $\mathrm{R}^{2}$ value does not decrease and no collinearity/multicollinearity is detected with other covariates in the multivariable meta-regression model.

In practice, only univariate meta-regression analyses could be satisfactorily conducted. Multivariate analyses were not possible due to a lack of data and given that there were not variables identified that modified the intervention effect within each stage of the analysis.

Results of the univariate meta-regression analyses were as follows, with variables displaying significant associations in bold.

\section{Stage 2:}

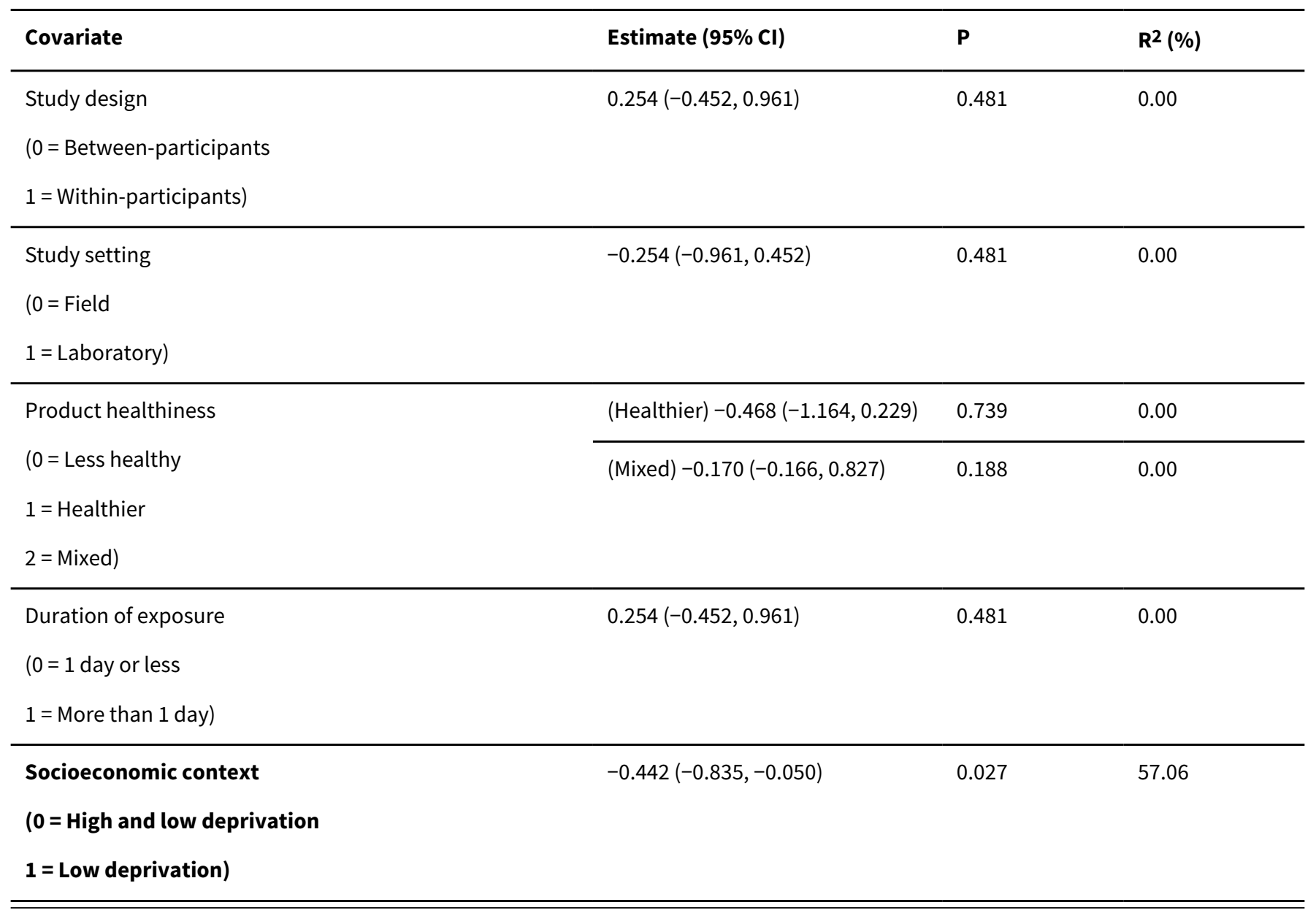

Altering the availability or proximity of food, alcohol, and tobacco products to change their selection and consumption (Review) 


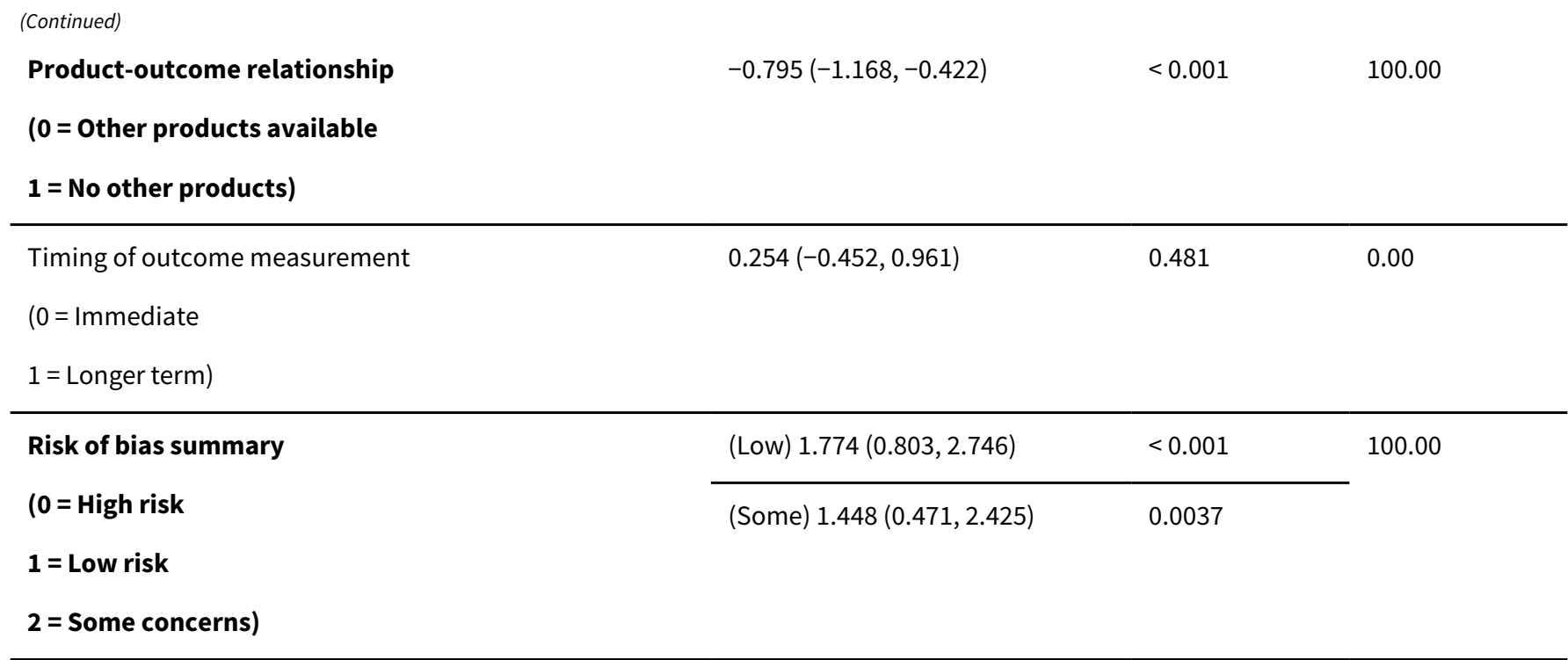

Stage 3:

\begin{tabular}{llll}
\hline Covariate & Estimate $(95 \% \mathrm{CI})$ & $\mathbf{P}$ & $\mathbf{R}^{\mathbf{2}}$ \\
\hline Absolute difference in proximity & $-0.0011(-0.0017,-0.0005)$ & $<0.001$ & 100.00 \\
\hline
\end{tabular}

Stage 4:

\begin{tabular}{llll}
\hline Covariate & Estimate $(95 \%$ CI) & P & $\mathbf{R}^{\mathbf{2}}$ \\
\hline Percentage of female participants & $0.0068(-0.0004,0.0140)$ & 0.063 & 6.13 \\
\hline
\end{tabular}

WHAT'S NEW

\begin{tabular}{lll}
\hline Date & Event & Description \\
\hline 4 September 2019 & $\begin{array}{l}\text { New citation required but conclusions } \\
\text { have not changed }\end{array}$ & $\begin{array}{l}\text { Changed to open access. No other changes made to the text of } \\
\text { the review. }\end{array}$ \\
\hline
\end{tabular}

\section{CONTRIBUTIONS OF AUTHORS}

Draft the protocol: all authors

Develop a search strategy: GJH, IS

Search for trials: GJH, PC, IS

Obtain copies of trials: GJH, PC, IS

Select studies for inclusion: GJH, PC, IS, DO

Extract data from studies: GJH, PC, SEK 
Enter data into Review Manager 5: GJH, PC, SEK

Carry out the analysis: SA, JPTH

Interpret the analysis: all authors

Draft the final review: all authors

\section{DECLARATIONS OF INTEREST}

Gareth Hollands declares no financial or other conflicts of interest.

Patrice Carter declares no financial or other conflicts of interest.

Sumayya Anwer declares no financial or other conflicts of interest.

Sarah King declares no financial or other conflicts of interest.

Susan Jebb declares no financial or other conflicts of interest.

David Ogilvie declares no financial or other conflicts of interest.

Ian Shemilt declares no financial or other conflicts of interest.

Julian Higgins declares no financial or other conflicts of interest.

Theresa Marteau declares no financial or other conflicts of interest.

\section{SOURCES OF SUPPORT}

\section{Internal sources}

- King's College London, UK.

Database access

- University of Cambridge, UK.

Computer provision, database access

- University of Bristol, UK.

Computer provision

- University College London, UK.

Computer provision

\section{External sources}

- Department of Health Policy Research Programme in England ((PR-UN-0409-10109) Policy Research Unit in Behaviour and Health), UK. Funding for the protocol

\section{DIFFERENCES BETWEEN PROTOCOL AND REVIEW}

1. Methods - Types of studies: We excluded randomised studies that had only a single participating site with site-level data in the intervention and/or the comparator group because this would result in the treatment effect being completely confounded with the site characteristics.

\section{NOTES}

This Review contains articles on which Brian Wansink (John Dyson Professor of Marketing at Cornell University) was an author, who is known to have committed academic misconduct (https://www.bmj.com/content/363/bmj.k4309). Seventeen of his articles have been retracted as of 9th August 2019 (http://retractiondatabase.org/RetractionSearch.aspx\#?auth\%3dWansink). None of the 17 retracted articles authored by Wansink have been included in this Cochrane Review. The results and conclusions of the review are therefore not affected. However, four articles on which Wansink is an author, and which have not been retracted, were included in this review, and two of these four articles contributed data to the primary meta-analyses. These retractions introduce additional uncertainty regarding the veracity of other studies Wansink has authored, including those contributing to this review. Should any study included in this review be retracted, we will withdraw that study's data from updated meta-analyses conducted as part of future updates of this Cochrane review. 


\section{N D EX TERMS}

\section{Medical Subject Headings (MeSH)}

${ }^{\star}$ Food Supply; *Public Health; Alcoholic Beverages ["supply \& distribution]; Environment; Noncommunicable Diseases [”prevention \& control]; Restaurants; Schools; Tobacco Products [*supply \& distribution]; Workplace

\section{MeSH check words}

Humans 